TRANSACTIONS OF THE

AMERICAN MATHEMATICAL SOCIETY

Volume 353, Number 6, Pages 2309-2346

S 0002-9947(00)02694-5

Article electronically published on November 29, 2000

\title{
GOOD IDEALS IN GORENSTEIN LOCAL RINGS
}

\author{
SHIRO GOTO, SIN-ICHIRO IAI, AND KEI-ICHI WATANABE
}

\begin{abstract}
Let $I$ be an m-primary ideal in a Gorenstein local ring $(A, \mathfrak{m})$ with $\operatorname{dim} A=d$, and assume that $I$ contains a parameter ideal $Q$ in $A$ as a reduction. We say that $I$ is a good ideal in $A$ if $G=\sum_{n>0} I^{n} / I^{n+1}$ is a Gorenstein ring with a $(G)=1-d$. The associated graded ring $G$ of $I$ is a Gorenstein ring with $\mathrm{a}(G)=-d$ if and only if $I=Q$. Hence good ideals in our sense are good ones next to the parameter ideals $Q$ in $A$. A basic theory of good ideals is developed in this paper. We have that $I$ is a good ideal in $A$ if and only if $I^{2}=Q I$ and $I=Q: I$. First a criterion for finite-dimensional Gorenstein graded algebras $A$ over fields $k$ to have nonempty sets $\mathcal{X}_{A}$ of good ideals will be given. Second in the case where $d=1$ we will give a correspondence theorem between the set $\mathcal{X}_{A}$ and the set $\mathcal{Y}_{A}$ of certain overrings of $A$. A characterization of good ideals in the case where $d=2$ will be given in terms of the goodness in their powers. Thanks to Kato's Riemann-Roch theorem, we are able to classify the good ideals in two-dimensional Gorenstein rational local rings. As a conclusion we will show that the structure of the set $\mathcal{X}_{A}$ of good ideals in $A$ heavily depends on $d=\operatorname{dim} A$. The set $\mathcal{X}_{A}$ may be empty if $d \leq 2$, while $\mathcal{X}_{A}$ is necessarily infinite if $d \geq 3$ and $A$ contains a field. To analyze this phenomenon we shall explore monomial good ideals in the polynomial ring $k\left[X_{1}, X_{2}, X_{3}\right]$ in three variables over a field $k$. Examples are given to illustrate the theorems.
\end{abstract}

\section{INTRODUCTION}

In this paper we study a certain class of $\mathfrak{m}$-primary ideals in a Gorenstein local ring in connection with the Gorensteinness of graded rings associated to those ideals.

Let $A$ be a Gorenstein local ring with the maximal ideal $\mathfrak{m}$ and $d=\operatorname{dim} A$. Let $I$ denote an $\mathfrak{m}$-primary ideal in $A$, and assume that $I$ contains a parameter ideal $Q=\left(a_{1}, a_{2}, \ldots, a_{d}\right)$ of $A$ as a reduction. The latter assumption is always satisfied if the field $A / \mathfrak{m}$ is infinite $\left([\mathrm{NR}]\right.$ ). Let $\mathrm{r}_{Q}(I)=\min \left\{n \geq 0 \mid I^{n+1}=Q I^{n}\right\}$ be the reduction number of $I$ with respect to $Q$. We define

$$
\begin{aligned}
& \mathrm{R}(I)=A[I t] \subseteq A[t], \\
& \mathrm{R}^{\prime}(I)=A\left[I t, t^{-1}\right] \subseteq A\left[t, t^{-1}\right], \\
& \mathrm{G}(I)=\mathrm{R}^{\prime}(I) / t^{-1} \mathrm{R}^{\prime}(I)
\end{aligned}
$$

(here $t$ denotes an indeterminate over $A$ ), and call them respectively the Rees algebra, the extended Rees algebra, and the associated graded ring of $I$. We put

Received by the editors July 25, 1999.

2000 Mathematics Subject Classification. Primary 13A30; Secondary 13H10.

Key words and phrases. Rees algebra, associated graded ring, Cohen-Macaulay ring, Gorenstein ring, a-invariant.

The first and third authors are supported by the Grant-in-Aid for Scientific Researches in Japan (C(2), No. 11640049 and 10640042, respectively). 
$G=\mathrm{G}(I), r=\mathrm{r}_{Q}(I)$, and $\mathfrak{M}=\mathfrak{m} G+G_{+}$. Let $\left[\mathrm{H}_{\mathfrak{M}}^{d}(G)\right]_{n}$ denote the homogeneous component of degree $n$ in the $d \underline{\text { th }}$ local cohomology module $\mathrm{H}_{\mathfrak{M}}^{d}(G)$ of $G$ with respect to $\mathfrak{M}$. Let

$$
\mathrm{a}(G)=\max \left\{n \in \mathbb{Z} \mid\left[\mathrm{H}_{\mathfrak{M}}^{d}(G)\right]_{n} \neq(0)\right\}
$$

(GW, (3.1.4)]). Then we say that our ideal $I$ is $\operatorname{good}$ if $G$ is a Gorenstein ring and $\mathrm{a}(G)=1-d$. This condition is equivalent to saying that $I^{2}=Q I$ and $I=Q: I$ (Proposition (2.2)). We denote by $\mathcal{X}_{A}$ the set of good ideals in $A$. The Rees algebra $\mathrm{R}(I)$ is a Cohen-Macaulay (resp. Gorenstein) ring if $I \in \mathcal{X}_{A}$ and $d \geq 2$ (resp. $d=3$ ) ([GS (1.1), (3.10)], [I, (3.1)]). Also, in the case where $A$ is a regular local ring with $\operatorname{dim} A=3$, we have $I=Q$ or $I \in \mathcal{X}_{A}$, once $\mathrm{G}(I)$ is a Gorenstein ring ([HHR $)$.

As is well-known, provided $G$ is a Cohen-Macaulay ring, we have a $(G)=r-d \geq$ $-d$, in which the equality a $(G)=-d$ holds true if and only if $I=Q$. Conversely, when this is the case, $G$ is a Gorenstein ring since it is a polynomial ring over $A / Q$. From this point of view the good ideals in our sense are good ones next to the parameter ideals $Q$ in $A$. However, although a fruitful and productive theory may be expected, a total investigation seems still lacking for good ideals in our sense. On the other hand, ideals possessing Gorenstein associated graded rings are rather rare. Therefore in the present research we must develop the theory, carefully exploring as many interesting examples as possible. Thus one of the main purposes of this paper is to clarify what kind of properties the good ideals really enjoy. Our other goal is to establish their ubiquity.

We are now ready to explain how this paper is organized. The contents are as follows.

1. Introduction

2. Preliminaries

3. Good ideals in finite-dimensional Gorenstein graded algebras over fields

4. A correspondence theorem when $\operatorname{dim} A=1$

5. Good ideals in one-dimensional reduced complete local rings of multiplicity 2

6. A characterization of good ideals when $\operatorname{dim} A=2$

7. Good ideals in two-dimensional Gorenstein rational local rings

8. Monomial good ideals in the polynomial ring $k\left[X_{1}, X_{2}, X_{3}\right]$ over a field $k$ when the case $\operatorname{dim} A \geq 3$

Contrary to our expectation, the results, including the existence of good ideals, heavily depend on the dimension $d$ of the base ring. First in Section 3 we shall analyze the starting case where $d=0$. A criterion for finite-dimensional Gorenstein graded algebras over a field $k$ to have nonempty sets $\mathcal{X}_{A}$ of good ideals will be given.

Theorem (3.1). Let $k$ be a field. Then the following conditions are equivalent.

(1) The field $k$ contains $\sqrt{\alpha}$ for any element $\alpha \in k$.

(2) Let $A=\sum_{n \geq 0} A_{n}$ be a finite-dimensional Gorenstein graded $k$-algebra with $k=A_{0}$. Then $\mathcal{X}_{A} \neq \varnothing$ if and only if $2 \mid \operatorname{dim}_{k} A$.

Therefore, when the field $k$ is algebraically closed, for any finite-dimensional Gorenstein graded $k$-algebra $A=\sum_{n \geq 0} A_{n}$ with $k=A_{0}$ we have that $\mathcal{X}_{A} \neq \varnothing$ if and only if $2 \mid \operatorname{dim}_{k} A$.

In Section 4 we shall study the case where $d=1$. We will give the following correspondence theorem between the set $\mathcal{X}_{A}$ and the set $\mathcal{Y}_{A}$ of certain overrings of $A$. Let $\ell_{A}(*)$ denote the length. 
Theorem (4.2). Assume $\operatorname{dim} A=1$ and let $K=\mathrm{Q}(A)$ be the total quotient ring of $A$. We denote by $\mathcal{Y}_{A}$ the set of Gorenstein $A$-subalgebras $C$ of $K$ such that $C \supsetneq A$ but the A-module $C$ is finitely generated. Then there is a one-to-one correspondence between the sets $\mathcal{X}_{A}$ and $\mathcal{Y}_{A}$, which sends each $I \in \mathcal{X}_{A}$ to $\operatorname{End}_{A} I=I:_{K} I$ and takes back each $C \in \mathcal{Y}_{A}$ to $\operatorname{Hom}_{A}(C, A)=A:_{K} C$. The correspondence reverses the inclusion, and one has the equality

$$
\ell_{A}(C / A)=\ell_{A}\left(A / A:_{K} C\right)
$$

for all $C \in \mathcal{Y}_{A}$.

One of the consequences of Theorem (4.2) shows that good ideals $I$ and $J$ in $A$ must coincide, once $I \cong J$ as $A$-modules (Corollary (4.3)). Hence the set $\mathcal{X}_{A}$ is finite if $A$ has finite CM-representation type. 2 .

In Section 5 we shall study one-dimensional Gorenstein local rings of multiplicity

Theorem (5.1). Let $A$ be a one-dimensional reduced complete local ring with $\mathrm{e}(A)=2$ and let $B=\bar{A}$ denote the normalization of $A$. Then:

(1) Every intermediate ring $A \subseteq C \subseteq B$ is a Gorenstein ring.

(2) $\sharp \mathcal{X}_{A}=\ell_{A}(B / A)$.

(3) The set $\mathcal{X}_{A}$ is totally ordered with respect to inclusion.

Hence there is a unique chain $A=C_{0} \subseteq C_{1} \subseteq \cdots \subseteq C_{n}=B$ of rings between $A$ and $B$, where $n=\ell_{A}(B / A)$.

Unless $\widehat{A}$ is reduced the set $\mathcal{X}_{A}$ is no longer finite, even though $A$ has multiplicity 2 (Example (5.7)).

In Section 6 we will study the case where $d=2$. The following characterization of good ideals will be given, in which $\mathrm{K}_{S}$ denotes for a Noetherian graded ring $S$ the graded canonical module. Let $\mu_{A}(*)$ stand for the number of generators.

Theorem (6.1). Let $\operatorname{dim} A=2$. Let $I$ be an $\mathfrak{m}$-primary ideal in $A$ and assume that $I$ contains a parameter ideal $Q$ as a reduction. Then the following nine conditions are equivalent to each other.

(1) $I \in \mathcal{X}_{A}$.

(2) $I^{\ell} \in \mathcal{X}_{A}$ for all $\ell \geq 1$.

(3) $I^{2}=Q I$ and $I^{\ell} \in \mathcal{X}_{A}$ for some $\ell \geq 1$.

(4) The Hilbert function $\ell_{A}\left(A / I^{n+1}\right)$ is a polynomial in $n$ for all $n \geq 0$, and $I^{\ell} \in \mathcal{X}_{A}$ for some $\ell \geq 1$.

(5) $\mathrm{G}(I)$ is a Cohen-Macaulay ring, and $I^{\ell} \in \mathcal{X}_{A}$ for some $\ell \geq 1$.

(6) $I^{n}=Q^{n}: I$ for all $n \in \mathbb{Z}$.

(7) $\ell_{A}\left(A / I^{n}\right)=n^{2} \ell_{A}(A / I)$ for all $n \geq 1$.

(8) $\mathrm{R}(I)$ is a Cohen-Macaulay ring and $\mathrm{K}_{\mathrm{R}(I)} \cong \mathrm{R}(I)_{+}$as graded $\mathrm{R}(I)$-modules.

(9) $\mathrm{R}^{\prime}(I)$ is a Gorenstein ring and $\mathrm{K}_{\mathrm{R}^{\prime}(I)} \cong \mathrm{R}^{\prime}(I)$ as graded $\mathrm{R}^{\prime}(I)$-modules.

When this is the case, the equality

$$
\mu_{A}\left(I^{n}\right)=n \mu_{A}(I)-n+1
$$

holds true for all $n \geq 1$.

Here we note that conditions (1) and (2) in Theorem (6.1) are not equivalent to each other unless $d=2$. In the case where $d=1$ we actually have that $\ell=1$ if 
$\ell \geq 1$ and $I^{\ell} \in \mathcal{X}_{A}$ (Corollary (4.5)). Even though $I^{\ell} \in \mathcal{X}_{A}$ for all $\ell \gg 0$, the ideal $I$ is not necessarily a good ideal in $A$ (Example (6.12)). However, if $I^{\ell} \in \mathcal{X}_{A}$ for some $\ell \geq 1$, then the powers $I^{n}$ of $I$ are good ideals in $A$ for all $n \geq N+1$, where $N$ denotes the least integer $N \geq 0$ such that the Hilbert function $\ell_{A}\left(A / I^{n+1}\right)$ of $I$ is a polynomial in $n$ for all $n \geq N$ (Theorem (6.4)).

We say that a two-dimensional Noetherian local ring $R$ is rational if $R$ is normal and there exists a desingularization $X \rightarrow \operatorname{Spec} R$ with $\mathrm{H}^{1}\left(X, \mathcal{O}_{X}\right)=(0)([\mathrm{L} 3])$. In the case where our Gorenstein local ring $A$ is rational, the theory of good ideals is closely related to that of adjoints $\widetilde{I}$ of ideals $I$ in the sense of J. Lipman ([L4). He proved that the equality $\widetilde{I}=Q: I$ holds true for any $\mathfrak{m}$-primary integrally closed ideal $I$ in $A$ with $Q$ a minimal reduction, if $A$ is a two-dimensional Gorenstein rational local ring. Therefore $I \in \mathcal{X}_{A}$ if and only if $\widetilde{I}=I$ (Remark (6.19)). In addition, thanks to Kato's Rieman-Roch theorem [K], we are able to classify the good ideals in two-dimensional Gorenstein excellent rational local rings $A$. Let $f: X \rightarrow \operatorname{Spec} A$ be a desingularization and let $I$ be an $\mathfrak{m}$-primary ideal in $A$. Then we say that $I$ is represented on $X$ if $I \mathcal{O}_{X}$ is invertible. With this notation we shall prove in Section 7 the following.

Theorem (7.8). Let $A$ be a two-dimensional Gorenstein excellent rational local ring. Let $I$ be an $\mathfrak{m}$-primary ideal in $A$ and assume that $I$ contains a parameter ideal $Q$ in $A$ as a reduction. Then $I$ is a good ideal in $A$ if and only if $I$ is an integrally closed ideal represented on the minimal resolution of Spec $A$.

According to an argument of Huneke and Swanson [HS], in the case where $\operatorname{dim} A=2$ we have $\operatorname{pd}_{A} A / I=\infty$ for all $I \in \mathcal{X}_{A}$ (Proposition (6.14)). Consequently, $\mathcal{X}_{A}=\varnothing$ if $A$ is a regular local $\operatorname{ring}$ with $\operatorname{dim} A \leq 2$ (cf. Proposition (7.5) also). However, if $\operatorname{dim} A \geq 3$ and $A$ contains a field, the set $\mathcal{X}_{A}$ is never empty and the set $\mathcal{X}_{A}$ is actually infinite (Proposition (8.1)). This assertion readily follows from the analysis of monomial good ideals in the polynomial ring $R=k\left[X_{1}, X_{2}, X_{3}\right]$ over a field $k$. To explicitly state our results on monomial good ideals in $R$, let $a_{1}, a_{2}, a_{3} \geq 1$ be integers and $Q=\left(X_{1}^{a_{1}}, X_{2}^{a_{2}}, X_{3}^{a_{3}}\right)$. We denote by $\mathcal{X}_{Q}$, or simply by $\mathcal{X}_{\left(a_{1}, a_{2}, a_{3}\right)}$, the set of ideals $J$ in $R$ which are generated by monomials in $X_{1}, X_{2}, X_{3}$ and such that $J \supseteq Q, J^{2}=Q J$, and $J=Q: J$. Then clearly $J R_{\mathfrak{M}} \in \mathcal{X}_{R_{\mathfrak{M}}}$ for all $J \in \mathcal{X}_{\left(a_{1}, a_{2}, a_{3}\right)}$, where $\mathfrak{M}=\left(X_{1}, X_{2}, X_{3}\right)$. Moreover, because $a_{i}=b_{i}$ for all $i=1,2,3$ if $\mathcal{X}_{\left(a_{1}, a_{2}, a_{3}\right)} \cap \mathcal{X}_{\left(b_{1}, b_{2}, b_{3}\right)} \neq \varnothing$ (Corollary (8.9)), we see that $\mathcal{X}_{R_{\mathfrak{M}}}$ is infinite, and hence so is the set $\mathcal{X}_{k\left[\left[X_{1}, X_{2}, X_{3}\right]\right]}$, once $\mathcal{X}_{\left(a_{1}, a_{2}, a_{3}\right)} \neq \varnothing$ for infinitely many vectors $\left(a_{1}, a_{2}, a_{3}\right)$ with the $a_{i}$ 's $\geq 1$. And in Section 8 we will prove the following.

Theorem (8.25). Let $a_{1}, a_{2}, a_{3} \geq 1$ be integers. Then $\mathcal{X}_{\left(a_{1}, a_{2}, a_{3}\right)}=\varnothing$ if and only if one of the following conditions is satisfied.

(1) $\left\{a_{1}, a_{2}, a_{3}\right\} \ni 1$.

(2) $2 \nmid a_{1} a_{2} a_{3}$.

(3) $\left(a_{1}, a_{2}, a_{3}\right)=(2,2$, odd $),(2$, odd, 2$)$, or $($ odd $, 2,2)$.

Our proof of Theorem (8.25) will turn into arithmetic, with a series of preliminary steps, and the most interesting consequence is the following.

Corollary (8.27). Suppose that $\min \left\{a_{1}, a_{2}, a_{3}\right\} \geq 3$. Then $\mathcal{X}_{\left(a_{1}, a_{2}, a_{3}\right)} \neq \varnothing$ if and only if $2 \mid a_{1} a_{2} a_{3}$.

At this moment we do not know whether the set $\mathcal{X}_{A}$ is still infinite if $\operatorname{dim} A \geq 3$ but $A$ contains no field. But a more urgent open problem is the question of when 
$\mathcal{X}_{A}=\varnothing$. We suspect that $\mathcal{X}_{A}=\varnothing$ if and only if $A$ is a regular local ring with $\operatorname{dim} A \leq 2$, provided that $\operatorname{dim} A \geq 1$.

Now before entering into details, let us mention again our standard notation, which we shall maintain throughout this paper. Let $(A, \mathfrak{m})$ be a Gorenstein local ring and $d=\operatorname{dim} A$. Unless otherwise specified, we denote by $I$ an $\mathfrak{m}$-primary ideal in $A$ containing a parameter ideal $Q=\left(a_{1}, a_{2}, \ldots, a_{d}\right)$ of $A$ as a reduction. Let $r=\mathrm{r}_{Q}(I)$. We denote by $\mu_{A}(*)$ the number of generators and by $\ell_{A}(*)$ the length.

Let $S=\bigoplus_{n \in \mathbb{Z}} S_{n}$ be a Noetherian graded ring and assume that $S$ contains a unique graded maximal ideal $\mathfrak{M}$. We denote by $\mathrm{H}_{\mathfrak{M}}^{i}(*)(i \in \mathbb{Z})$ the $i \frac{t h}{\text { local }}$ cohomology functor of $S$ with respect to $\mathfrak{M}$. For each graded $S$-module $E$ and $n \in \mathbb{Z}$, let $\left[\mathrm{H}_{\mathfrak{M}}^{i}(E)\right]_{n}$ denote the homogeneous component of the graded $S$-module $\mathrm{H}_{\mathfrak{M}}^{i}(E)$ of degree $n$. If $S_{n}=(0)$ for all $n<0$ and $E$ is a finitely generated graded $S$-module, we have $\left[\mathrm{H}_{\mathfrak{M}}^{i}(E)\right]_{n}=(0)$ for all $n \gg 0$ and $i \in \mathbb{Z}$. We put $\mathrm{a}(E)=\sup \left\{n \in \mathbb{Z} \mid\left[\mathrm{H}_{\mathfrak{M}}^{s}(E)\right]_{n} \neq(0)\right\}$ with $s=\operatorname{dim}_{S} E$, and call it the a-invariant of $E$ ([GW, (3.1.4)]). For each $n \in \mathbb{Z}$ let $E(n)$ stand for the graded $S$-module whose underlying $S$-module coincides with that of $E$ and whose grading is given by $[E(n)]_{i}=E_{n+i}$ for all $i \in \mathbb{Z}$. We denote by $\mathrm{K}_{S}$ the graded canonical module of $S$, if it exists. We shall freely refer to $[\mathrm{BH}], \mathrm{GN}], \mathrm{HK}]$, and [HIO for the details on canonical modules.

\section{Preliminaries}

In this section we shall summarize some basic results on good ideals for later use in this paper. Let $I$ be an $\mathfrak{m}$-primary ideal in $A$, and assume that $I$ contains a parameter ideal $Q=\left(a_{1}, a_{2}, \ldots, a_{d}\right)$ of $A$ as a reduction. Hence $Q$ is a minimal reduction of $I$, and the elements $a_{1}, a_{2}, \cdots, a_{d}$ form a part of a minimal system of generators for $I$ ([NR or $[\mathrm{S} 2$, Ch.2, Theorem 1.7]). We say that $I$ is a good ideal in $A$ if $\mathrm{G}(I)$ is a Gorenstein ring with a $(\mathrm{G}(I))=1-d$. Let $\mathcal{X}_{A}$ denote the set of good ideals in $A$.

Let $\widehat{A}$ denote the $\mathfrak{m}$-adic completion of $A$ and let $\mathfrak{a}^{e}=\mathfrak{a} \widehat{A}$ be the extension of an ideal $\mathfrak{a}$ in $\widehat{A}$. Then the correspondence $\mathfrak{a} \mapsto \mathfrak{a}^{e}$ defines a bijection between $\mathfrak{m}$-primary ideals in $A$ and $\mathfrak{m}^{e}$-primary ideals in $\widehat{A}$. Therefore, since $\mathrm{G}\left(I^{e}\right)=\mathrm{G}(I)$ for all $I \in \mathcal{X}_{A}$, the correspondence $I \mapsto I^{e}$ gives rise to a bijection between the sets $\mathcal{X}_{A}$ and $\mathcal{X}_{\widehat{A}}$, so that we have

Lemma (2.1). $\sharp \mathcal{X}_{A}=\sharp \mathcal{X}_{\widehat{A}}$.

For each $I \in \mathcal{X}_{A}$ let $\mathrm{t}(A / I)=\ell_{A}\left((0):_{A / I} \mathfrak{m}\right)$ be the Cohen-Macaulay type of $A / I$ and let $\mathrm{e}_{I}(A)=\mathrm{e}_{I}^{0}(A)$ denote the multiplicity of $A$ with respect to $I$.

Proposition (2.2). Let $I$ be an $\mathfrak{m}$-primary ideal in $A$ and assume that I contains a parameter ideal $Q=\left(a_{1}, a_{2}, \ldots, a_{d}\right)$ of $A$ as a reduction. Then the following conditions are equivalent.

(1) $I \in \mathcal{X}_{A}$.

(2) $I^{2}=Q I$ and $I=Q: I$.

(3) $I^{2}=Q I$ and $\ell_{A}(A / I)=\frac{1}{2} \ell_{A}(A / Q)$.

(4) $I^{3} \subseteq Q^{2}$ and $I=Q: I$.

(5) $R^{\prime}:=\mathrm{R}^{\prime}(I)$ is a Gorenstein ring with $\mathrm{K}_{R^{\prime}} \cong R^{\prime}(2-d)$.

If $d \geq 1$, we may add the following.

(6) $I^{n}=Q^{n}: I$ for all $n \in \mathbb{Z}$. 
When this is the case, we have $\mathrm{t}(A / I)=\mu_{A}(I / Q)=\mu_{A}(I)-d \geq 1$ and $\mathrm{e}_{I}(A)=$ $2 \ell_{A}(A / I)$.

Proof. (1) $\Leftrightarrow(2)$ We put $G=\mathrm{G}(I)$ and $r=\mathrm{r}_{Q}(I)$. Let $f_{i}=a_{i} t(1 \leq i \leq d)$. To prove the equivalence we may assume $G$ is a Cohen-Macaulay ring, since $G$ is Cohen-Macaulay if $I^{2}=Q I([\overline{V V}, 3.1])$. Therefore the sequence $f_{1}, f_{2}, \ldots, f_{d}$ is $G$-regular, so that by [VV] 2.1] we have the isomorphism

$$
G /\left(f_{1}, f_{2}, \ldots, f_{d}\right) G \cong \mathrm{G}(I / Q)
$$

of graded $A$-algebras. Hence a $(G)=r-d$ by [GW, (3.1.6)], because $I^{r} \nsubseteq Q$ (note that $Q \cap I^{n}=Q I^{n-1}$ for all $n \in \mathbb{Z}$ ). Thus $r=1$ if $I \in \mathcal{X}_{A}$. So we may furthermore assume $r=1$, whence $\mathrm{G}(I / Q)=A / I \oplus I / Q$ with $I / Q \neq(0)$. As $(I / Q)^{2}=(0)$ in $\mathrm{G}(I / Q)$, the ring $\mathrm{G}(I / Q)=A / I \oplus I / Q$ is the idealization of $I / Q$ over $A / I$, whence it is a Gorenstein ring if and only if $\mathrm{K}_{A / I} \cong I / Q\left(\left[\mathbb{R}_{]}\right)\right.$. We notice that $\ell_{A}\left((0)::_{I / Q} \mathfrak{m}\right)=1$, since $I / Q$ is an ideal in the Artinian Gorenstein local ring $A / Q$. Hence $\mathrm{K}_{A / I} \cong I / Q$ if and only if $I / Q$ is a faithful $A / I$-module ([BH, 3.3.13]). Therefore by (2.3), $G$ is a Gorenstein ring if and only if $I=Q: I$. When this is the case, we have $\mathrm{t}(A / I)=\mu_{A}(I / Q)=\mu_{A}(I)-d \geq 1$, since $\mathrm{t}(A / I)=\mu_{A}\left(\mathrm{~K}_{A / I}\right)([\mathrm{BH}]$ 3.3.11]) and since the elements $a_{1}, a_{2}, \ldots, a_{d}$ form a part of a minimal system of generators for the ideal $I([\mathrm{~S} 2, \mathrm{Ch} .2$, Theorem 1.7]).

$(2) \Leftrightarrow(3)$ Because $[Q: I] / Q \cong \operatorname{Hom}_{A / Q}(A / I, A / Q)$ and $A / Q$ is a Gorenstein ring, we have $\ell_{A}([Q: I] / Q)=\ell_{A}(A / I)$. On the other hand, as $I \subseteq Q: I$, we see that $I=Q: I$ if and only if $\ell_{A}(I / Q)=\ell_{A}([Q: I] / Q)$. Hence $I=Q: I$ if and only if $\ell_{A}(I / Q)=\ell_{A}(A / I)$, which is equivalent to saying that $\ell_{A}(A / Q)=2 \ell_{A}(A / I)$. As $\mathrm{e}_{I}(A)=\ell_{A}(A / Q)$, we have $\mathrm{e}_{I}(A)=2 \ell_{A}(A / I)$ for all $I \in \mathcal{X}_{A}$.

$(2) \Rightarrow(4)$ This is clear.

(4) $\Rightarrow(2)$ Since $I^{2} \subseteq Q$, we may assume $d \geq 1$. Let $x \in I^{2}$ and write $x=$ $\sum_{i=1}^{d} c_{i} a_{i}$ with $c_{i} \in A$. Then for any $z \in I$, since $z x=\sum_{i=1}^{d}\left(z c_{i}\right) a_{i} \in I^{3} \subseteq Q^{2}$, we have $z c_{i} \in Q$ for all $1 \leq i \leq d$. Thus $c_{i} \in Q: I=I$, so that $x \in Q I$ and hence $I^{2}=Q I$.

$(1) \Leftrightarrow(5)$ Let $u=t^{-1}$ and $a=\mathrm{a}(G)$. Then, since $G=R^{\prime} / u R^{\prime}$ and $u$ is not a zero divisor of $R^{\prime}$, we may assume that $G$ and $R^{\prime}$ are Gorenstein rings. As $\mathrm{K}_{G} \cong\left[\mathrm{K}_{R^{\prime}} / u \mathrm{~K}_{R^{\prime}}\right](-1)$ and $\mathrm{K}_{G} \cong G(a)$ (GW, (3.1.4)]), the $R^{\prime}$-module $\mathrm{K}_{R^{\prime}}$ is generated by a single element with degree $-(a+1)$, whence $\mathrm{K}_{R^{\prime}} \cong R^{\prime}(a+1)$. Thus $\mathrm{K}_{R^{\prime}} \cong R^{\prime}(2-d)$ if and only if $a=1-d$.

Now let $d \geq 1$.

(6) $\Rightarrow$ (4) We have $I^{3} \subseteq Q^{2}$, since $I^{2}=Q^{2}: I$.

$(2) \Rightarrow(6)$ We may assume $n \geq 2$. It suffices to show that $I^{n} \supseteq Q^{n}: I$. Let $x \in Q^{n}: I$. Then, since $a_{1} x \in Q^{n} \cap a_{1} A=a_{1} Q^{n-1}$, we have $x \in Q^{n-1}$. Let $x=\sum_{|\alpha|=n-1} c_{\alpha} a^{\alpha}$ with $c_{\alpha} \in A$ (here $|\alpha|:=\sum_{i=1}^{d} \alpha_{i}$ and $a^{\alpha}:=a_{1}^{\alpha_{1}} a_{2}^{\alpha_{2}} \ldots a_{d}^{\alpha_{d}}$ for each $\alpha=\left(\alpha_{1}, \alpha_{2}, \ldots, \alpha_{d}\right)$ such that $\left.0 \leq \alpha_{i} \in \mathbb{Z}\right)$. Then, because $z x=$ $\sum_{|\alpha|=n-1}\left(z c_{\alpha}\right) a^{\alpha} \in Q^{n}$, we have $z c_{\alpha} \in Q$ for all $z \in I$, so that $c_{\alpha} \in Q: I=I$ for all $\alpha$. Thus $x \in Q^{n-1} I=I^{n}$, whence $I^{n}=Q^{n}: I$.

Corollary (2.4). Let $I \in \mathcal{X}_{A}$, and choose a parameter ideal $Q$ in $A$ so that $Q$ is a reduction of $I$. Let $J$ be an ideal in $A$, and assume that $J^{2} \subseteq Q$. Then $I=J$ if $I \subseteq J$

Proof. As $I \subseteq J$, we have $I=Q: I \supseteq Q: J \supseteq J$. Hence $I=J$.

Let $\overline{\mathfrak{a}}$ denote, for an ideal $\mathfrak{a}$ in $A$, the integral closure of $\mathfrak{a}$. 
Corollary (2.5). Let $A$ be a two-dimensional Gorenstein rational local ring. Then $\bar{I}=I$ for all $I \in \mathcal{X}_{A}$.

Proof. Choose a parameter ideal $Q$ in $A$ so that $Q$ is a reduction of $I$. Then $Q$ is a reduction of $\bar{I}$, whence $\bar{I}^{2}=Q \bar{I}([\overline{\mathrm{LT}}])$, and so $\bar{I}=I$ by $(2.4)$.

We close this section with the following remarks.

Remark (2.6). Let $I$ be an $\mathfrak{m}$-primary ideal in $A$ and assume that $I$ contains a parameter ideal $Q$ in $A$ as a reduction.

(1) Suppose $I \in \mathcal{X}_{A}$. Then $\mathrm{R}(I)$ is a Cohen-Macaulay (resp. Gorenstein) ring if $d \geq 2($ resp. $d=3)$.

(2) Suppose $\operatorname{dim} A=3$. Then $I \in \mathcal{X}_{A}$ if and only if $\mathrm{R}(I)$ is a Gorenstein ring.

(3) Suppose that $A$ is a regular local $\operatorname{ring}$ with $\operatorname{dim} A=3$. Then $I=Q$ or $I \in \mathcal{X}_{A}$, if $\mathrm{G}(I)$ is a Gorenstein ring.

Proof. For (1) and (2), see [GS, (1.1) and (3.10)] and [I, (3.1)].

(3) We have $\mathrm{a}(\mathrm{G}(I))=\mathrm{r}_{Q}(I)-3 \leq-2$ by [HHR]. Hence $\mathrm{r}_{Q}(I) \leq 1$, so that either $I=Q$ or $I \in \mathcal{X}_{A}$.

\section{Good ideals in Finite-Dimensional GORENSTEIN GRADED ALGEBRAS OVER FIELDS}

In this section we assume that $\operatorname{dim} A=0$. Therefore, for an ideal $I$ in $A$, by $(2.2)$ $I \in \mathcal{X}_{A}$ if and only if $I^{2}=(0)$ and $\ell_{A}(A)=2 \ell_{A}(I)$. Hence $2 \mid \ell_{A}(A)$ if $\mathcal{X}_{A} \neq \varnothing$. The converse is not true in general, as we shall show in the following.

Theorem (3.1). Let $k$ be a field. Then the following conditions are equivalent.

(1) The field $k$ contains $\sqrt{\alpha}$ for any element $\alpha \in k$.

(2) Let $A=\sum_{n \geq 0} A_{n}$ be a finite-dimensional Gorenstein graded $k$-algebra with $k=A_{0}$. Then $\mathcal{X}_{A} \neq \varnothing$ if and only if $2 \mid \operatorname{dim}_{k} A$.

We divide the proof of Theorem (3.1) into several steps. Let $k$ be a field and let $A=\sum_{i>0} A_{i}$ be a finite-dimensional Gorenstein graded $k$-algebra with $k=A_{0}$. We put $a=\mathrm{a}(A):=\max \left\{i \in \mathbb{Z} \mid A_{i} \neq(0)\right\}$ and choose $z \in A_{a}$ so that $A_{a}=k z$.

Let $\rho: A \stackrel{\pi}{\rightarrow} A_{a} \stackrel{\sigma}{\rightarrow} k$, where $\pi$ is the projection map and $\sigma$ is the $k$-isomorphism given by $\sigma(z)=1$. Then the pairing $\langle\rangle:, A \times A \rightarrow k$ defined by $\langle x, y\rangle=\rho(x y)$ is a non-degenerate symmetric bilinear form on $A$ and gives rise to the isomorphism $\varphi: A(a) \rightarrow A^{*}$ of graded $A$-modules, where $A^{*}=\operatorname{Hom}_{k}(A, k)$ and $\varphi(x)=\langle x, *\rangle$ for each $x \in A(a)$. Hence the pairing $A_{a-i} \times A_{i} \longrightarrow k,(x, y) \longmapsto\langle x, y\rangle$ is also perfect, and one has the equality $\operatorname{dim}_{k} A_{a-i}=\operatorname{dim}_{k} A_{i}$ for all $i \in \mathbb{Z}$. Therefore, if $a=2 b+1$, letting $I=\sum_{i>b+1} A_{i}$, we have $I^{2}=(0)$ and $\ell_{A}(I)=\operatorname{dim}_{k} I=\frac{1}{2} \operatorname{dim}_{k} A=\frac{1}{2} \ell_{A}(A)$. Thus $I \in \mathcal{X}_{A}$, and we get the following.

Proposition (3.2). $\mathcal{X}_{A} \neq \varnothing$ if $a=\mathrm{a}(A)$ is odd.

Assume that $2 \mid \operatorname{dim}_{k} A$ and $a=2 b(b \geq 1)$. Then $2 \mid \operatorname{dim}_{k} A_{b}, \operatorname{since} \operatorname{dim}_{k} A_{a-i}=$ $\operatorname{dim}_{k} A_{i}$ for all $i \in \mathbb{Z}$. We look at the perfect pairing $\langle\rangle:, A_{b} \times A_{b} \rightarrow k,(x, y) \mapsto$ $\langle x, y\rangle=\sigma(x y)$. Note that $\langle x, y\rangle=0$ in $k$ if and only if $x y=0$ in $A$, because $x y \in A_{2 b}=A_{a}$ for all $x, y \in A_{b}$. Choose a $k$-basis $\left\{\omega_{1}, \omega_{2}, \ldots, \omega_{n}\right\}\left(n=\operatorname{dim}_{k} A_{b}\right)$ 
of $A_{b}$ so that the symmetric $n \times n$ matrix $\mathcal{S}=\left[\left\langle\omega_{i}, \omega_{j}\right\rangle\right]$ has the following canonical form:

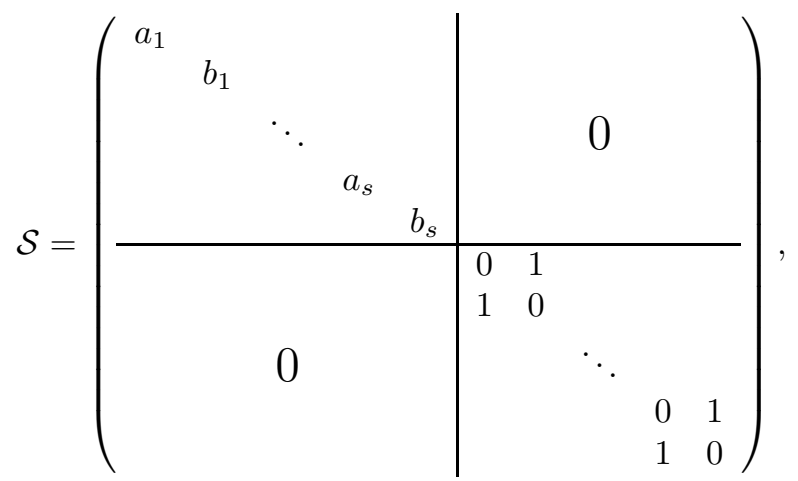

where $a_{i}, b_{i} \in k \backslash\{0\}$. Let $t$ be the number of the blocks $\left[\begin{array}{ll}0 & 1 \\ 1 & 0\end{array}\right]$ in $\mathcal{S}$. Hence $s+t=\frac{n}{2}$. We put $x_{i}=\omega_{2 i-1}, y_{i}=\omega_{2 i}(1 \leq i \leq s)$, and $f_{j}=\omega_{2(s+j)-1}(1 \leq j \leq t)$.

Proof of Theorem (3.1). (1) $\Rightarrow$ (2) Choose elements $\left\{c_{i}\right\}_{1 \leq i \leq s}$ in $k$ so that $c_{i}^{2}=$ $-\frac{a_{i}}{b_{i}}$ for $1 \leq i \leq s$. Let $I=\left(x_{i}+c_{i} y_{i} \mid 1 \leq i \leq s\right)+\left(f_{j} \mid 1 \leq j \leq t\right)+\sum_{i \geq b+1} A_{i}$. Then

Claim. $I \in \mathcal{X}_{A}$.

Proof of Claim. We put $z_{i}=x_{i}+c_{i} y_{i}(1 \leq i \leq s)$. Then, since

$$
\left\langle z_{i}, z_{i}\right\rangle=\left\langle x_{i}, x_{i}\right\rangle+c_{i}^{2}\left\langle y_{i}, y_{i}\right\rangle=a_{i}+c_{i}^{2} b_{i}=0,
$$

we have $z_{i} z_{j}=0$ in $A$ for all $1 \leq i, j \leq s$. Similarly, because $\left\langle f_{j}, f_{\ell}\right\rangle=\left\langle z_{i}, f_{j}\right\rangle=0$, we get $z_{i} f_{j}=f_{j} f_{\ell}=0$ for all $1 \leq i \leq s$ and $1 \leq j, \ell \leq t$. Thus $I^{2}=(0)$, as $A_{i}=(0)$ for $i \geq 2 b+1$. Hence $I \in \mathcal{X}_{A}$, as

$$
\ell_{A}(I)=\frac{n}{2}+\ell_{A}\left(\sum_{i \geq b+1} A_{i}\right)=\frac{1}{2} \ell_{A}(A) .
$$

$(2) \Rightarrow(1)$ Let $0 \neq \alpha \in k$ and look at the following ring

$$
A=k[X, Y] /\left(X^{2}+\alpha Y^{2}, X Y\right),
$$

where $k[X, Y]$ is the polynomial ring in two variables over $k$. Then $A$ is a finitedimensional Gorenstein graded $k$-algebra with $k=A_{0}$. Let $x, y$ denote respectively the reduction of $X, Y$ mod the ideal $\left(X^{2}+\alpha Y^{2}, X Y\right)$. Then the algebra $A$ contains $\left\{1, x, y, x^{2}=-\alpha y^{2}\right\}$ as a $k$-basis. Hence $\operatorname{dim}_{k} A=4$ and $\mathrm{a}(A)=2$. Consequently $\mathcal{X}_{A} \neq \varnothing$. Let us choose $I \in \mathcal{X}_{A}$. Then $\ell_{A}(I)=2$, and the ideal $I$ contains the socle $x^{2}=-\alpha y^{2}$ of $A$. Hence we may write $I=\left(f, x^{2}\right)$, where $f=a x+b y$ with $a, b \in k$. Then $f \neq 0$ but $f^{2}=a^{2} x^{2}+b^{2} y^{2}=0$ since $I^{2}=(0)$. Therefore $b^{2}=\alpha a^{2}$, whence $b / a=\sqrt{\alpha}$.

To illustrate Theorem (3.1) let us explore the following.

Example (3.4). Let $k[X, Y]$ be the polynomial ring in two variables over a field $k$ and let $A=k[X, Y] /\left(X^{n}-Y^{n}, X Y\right)(n \geq 2)$. Then $\sharp \mathcal{X}_{A} \leq 2$. More explicitly we have 
(1) $\mathcal{X}_{A}=\varnothing$ if and only if $n$ is even and $\alpha^{2} \neq-1$ for any $\alpha \in k$.

(2) $\sharp \mathcal{X}_{A}=1$ if and only if $n$ is odd, or $n$ is even and $\operatorname{ch} k=2$.

Proof. Since $X^{n}-Y^{n}, X Y$ is a regular sequence in $k[X, Y]$, the Hilbert series $\mathrm{H}(A, \lambda)=\sum_{i=0}^{\infty}\left(\operatorname{dim}_{k} A_{i}\right) \lambda^{i}$ of $A$ is given by

$$
\mathrm{H}(A, \lambda)=\frac{\left(1-\lambda^{n}\right)\left(1-\lambda^{2}\right)}{(1-\lambda)^{2}}=1+\sum_{i=1}^{n-1} 2 \lambda^{i}+\lambda^{n} .
$$

Hence $\operatorname{dim}_{k} A_{i}=2(1 \leq i \leq n-1)$ and $\operatorname{dim}_{k} A_{i}=1(i=0, n)$. So we have $\mathrm{a}(A)=n$ and $\ell_{A}(A)=\operatorname{dim}_{k} A=2 n$. Let $x, y$ denote respectively the reduction of $X, Y$ mod the ideal $\left(X^{n}-Y^{n}, X Y\right)$. First we consider the case where $n=2 m+1$ with $m \geq 1$. Let $J=\sum_{i \geq m+1}$. Then $\mathcal{X}_{A}=\{J\}$. In fact, $J \in \mathcal{X}_{A}$ by (3.2). Let $I \in \mathcal{X}_{A}$. We want to show that $I \subseteq J$. Assume the contrary, and choose $f \in I$ so that $f \notin J$. Let $f=\sum_{i=0}^{n} f_{i}$ with $f_{i} \in A_{i}$ and put $p=\min \left\{0 \leq i \leq n \mid f_{i} \neq 0\right\}$. Then $1 \leq p \leq m$. We write $f_{p}=a x^{p}+b y^{p}$ with $a, b \in k$. Then, since $f^{2}=0$, we get $f_{p}^{2}=a^{2} x^{2} p+b^{2} y^{2 p}=0$. As $2 p \leq 2 m<n$, we have $a=b=0$, whence $f_{p}=0$, which is absurd. Thus $I \subseteq J$, so that we have $I=J$ by (2.4). Hence $\mathcal{X}_{A}=\{J\}$.

Now assume $n=2 m$ with $m \geq 1$. Let $I \in \mathcal{X}_{A}$. Then $I \subseteq \sum_{i>m} A_{i}$ for the same reason as above. Hence $\sum_{i \geq m+1} A_{i} \subseteq(0): I=I$, so that $I=k f+\sum_{i>m+1} A_{i}$ for some $0 \neq f \in A_{m}$, because $\ell_{A}(I)=\frac{1}{2} \ell_{A}(A)=\ell_{A}\left(\sum_{i>m+1} A_{i}\right)+1$. We write $f=a x^{m}+b y^{m}$ with $a, b \in k$. Then, since $f^{2}=\left(a^{2}+b^{2}\right) x^{n}=0$, we have $a^{2}+b^{2}=0$. Hence $\left(\frac{b}{a}\right)^{2}=-1$ and $I=\left(x^{m}+\alpha y^{m}\right)+\sum_{i \geq m+1} A_{i}$, with $\alpha=\frac{b}{a}$. Consequently $\sharp \mathcal{X}_{A} \leq 2$. It is standard to check that $\left(x^{m}+\alpha y^{m}\right)+\sum_{i \geq m+1} A_{i}$ is a good ideal in $A$ for $\alpha=\sqrt{-1}$ in $k$. Hence $\mathcal{X}_{A}=\varnothing$ if and only if $\alpha^{2} \neq-1$ for any $\alpha \in k$. The set $\mathcal{X}_{A}$ is a singleton if and only if $\operatorname{ch} k=2$.

The above proof of Theorem (3.1) contains a little more information about the structure of $\mathcal{X}_{A}$ in the case where $k=\mathbb{R}$. Similarly as above, let $A=\sum_{i \geq 0} A_{i}$ be a finite-dimensional Gorenstein graded $\mathbb{R}$-algebra with $\mathbb{R}=A_{0}$. Assume that $a:=\mathrm{a}(A)=2 b$ and choose an $\mathbb{R}$-basis $\left\{\omega_{1}, \omega_{2}, \ldots, \omega_{n}\right\}$ of $A_{b}\left(n=\operatorname{dim}_{\mathbb{R}} A_{b}\right)$ so that the $n \times n$ symmetric matrix $\mathcal{S}=\left[\left\langle\omega_{i}, \omega_{j}\right\rangle\right]$ has the form

$$
\mathcal{S}=\left(\begin{array}{c|c}
I_{p} & 0 \\
\hline 0 & -I_{q}
\end{array}\right)
$$

with $p+q=n$, where $I_{r}$ denotes the identity matrix of rank $r$. Then we have the following.

Theorem (3.5). $\mathcal{X}_{A} \neq \varnothing$ if and only if $p=q$.

Proof. If $p=q$, then $2 \mid \operatorname{dim}_{\mathbb{R}} A$. Since $\left[\begin{array}{cc}1 & 0 \\ 0 & -1\end{array}\right]$ is similar to $\left[\begin{array}{ll}0 & 1 \\ 1 & 0\end{array}\right]$ over the field $\mathbb{R}$, we may assume that $s=0$ in the matrix given by (3.3). Hence the proof of Theorem (3.1) still works to show that $\mathcal{X}_{A} \neq \varnothing$. Conversely, suppose that $\mathcal{X}_{A} \neq \varnothing$ and choose $I \in \mathcal{X}_{A}$. Let $f_{1}, f_{2}, \ldots, f_{\ell} \quad\left(\ell=\frac{1}{2} \operatorname{dim}_{\mathbb{R}} A\right)$ be an $\mathbb{R}$-basis of $I$. Firstly notice that since the pairing $A_{a-i} \times A_{i} \longrightarrow k,(x, y) \longmapsto\langle x, y\rangle$ is perfect, for each $0 \leq i \leq b-1$ and for each $\mathbb{R}$-basis $\left\{x_{1}^{(i)}, x_{2}^{(i)}, \ldots, x_{n_{i}}^{(i)}\right\}\left(n_{i}=\operatorname{dim}_{\mathbb{R}} A_{i}\right)$ of $A_{i}$, we may choose an $\mathbb{R}$-basis $\left\{y_{1}^{(a-i)}, y_{2}^{(a-i)}, \ldots, y_{n_{i}}^{(a-i)}\right\}$ of $A_{a-i}$ so that $\left\langle x_{\alpha}^{(i)}, y_{\beta}^{(a-i)}\right\rangle=\delta_{\alpha, \beta}$ for all $1 \leq \alpha, \beta \leq n_{i}$. On the other hand, we have $\langle x, y\rangle=0$ for any $x \in A_{i}$ and $y \in A_{j}$ with $i+j \neq a$, since $\pi(x y)=0$. Thus, considering the $\mathbb{R}$-basis $\left\{\omega_{i}\right\}_{1 \leq i \leq n}$ together 
with $\left\{x_{\alpha}^{(i)}\right\}_{0 \leq i \leq b-1,1 \leq \alpha \leq n_{i}},\left\{y_{\alpha}^{(a-i)}\right\}_{0 \leq i \leq b-1,1 \leq \alpha \leq n_{i}}$ of the whole $\mathbb{R}$-space $A$, we find that the matrix $\mathcal{M}$ of the pairing $\langle\rangle:, A \times A \rightarrow k,(x, y) \mapsto\langle x, y\rangle=\rho(x y)$ with respect to this basis has the form

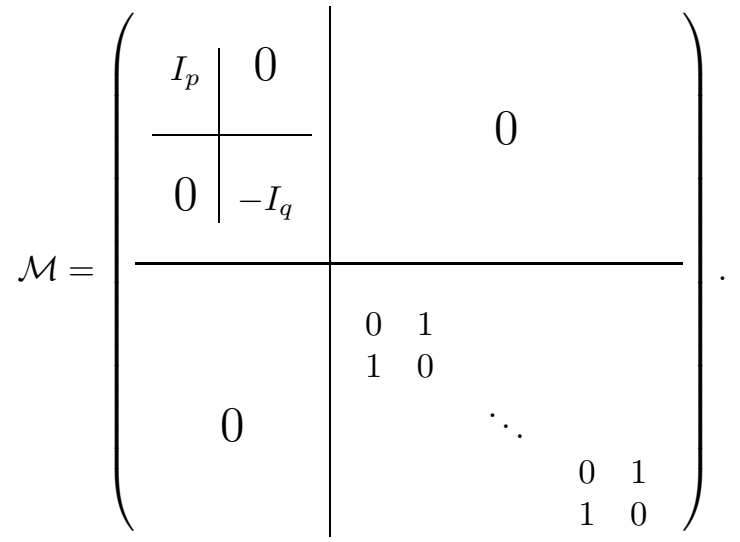

Since $\left[\begin{array}{ll}0 & 1 \\ 1 & 0\end{array}\right]$ is similar to $\left[\begin{array}{cc}1 & 0 \\ 0 & -1\end{array}\right]$, after a suitable change of bases together with renumbering we may assume that for some $\mathbb{R}$-basis $\left\{x_{1}, x_{2}, \ldots, x_{s}, y_{1}, y_{2}, \ldots, y_{t}\right\}$ of $A$ the matrix $\mathcal{M}$ has the form

$$
\mathcal{M}=\left(\begin{array}{c|c}
I_{s} & 0 \\
\hline 0 & -I_{t}
\end{array}\right),
$$

where $s=p+\frac{2 \ell-n}{2}, t=q+\frac{2 \ell-n}{2}$, and $2 \ell=\operatorname{dim}_{\mathbb{R}} A$. We want to show that $s=t$. For each $1 \leq i \leq \ell$ let us write $f_{i}=g_{i}+h_{i}$ with $g_{i} \in \sum_{j=1}^{s} \mathbb{R} x_{j}$ and $h_{i} \in \sum_{j=1}^{t} \mathbb{R} y_{j}$. Let $V=\sum_{i=1}^{\ell} \mathbb{R} g_{i}$ and $v=\operatorname{dim}_{\mathbb{R}} V$. Then $v \leq s$.

Claim. Let $f \in I$. Then $f=0$ if $f \in \sum_{j=1}^{t} \mathbb{R} y_{j}$.

Proof of Claim. Let $f=\sum_{j=1}^{t} a_{j} y_{j}$ with $a_{j} \in \mathbb{R}$. Then, since $f^{2}=0$, we have $\langle f, f\rangle=0$, so that $\sum_{j=1}^{t} a_{j}^{2}=0$. Thus $f=0$.

Now suppose that $\ell>s$. After renumbering, we may assume $\left\{g_{1}, g_{2}, \ldots, g_{v}\right\}$ is an $\mathbb{R}$-basis of $V$. Then $v \leq s<\ell$ and $g_{v+1} \in V$. We write $g_{v+1}=\sum_{i=1}^{v} c_{i} g_{i}$ with $c_{i} \in \mathbb{R}$, and let $f=f_{v+1}-\sum_{i=1} c_{i} f_{i}$. Then, since $f=h_{v+1}-\sum_{i=1}^{v} c_{i} h_{i}$, we have $f \in I \cap \sum_{j=1}^{t} \mathbb{R} y_{j}$. Hence by the above claim $f=0$, so that $f_{v+1}=\sum_{i=1}^{v} c_{i} f_{i}$. This is absurd, since $\left\{f_{i}\right\}_{1 \leq i \leq \ell}$ is an $\mathbb{R}$-basis of $I$. Hence $\ell \leq s$. Similarly we have $\ell \leq t$. Therefore $s=t=\ell$, because $2 \ell=\ell_{A}(A)=s+t$. Thus $p=q$.

Let us note the following example.

Example (3.6). Let $n \geq 1$ be an integer and let $R=k\left[X_{i}, Y_{i} \mid 1 \leq i \leq n\right]$ be the polynomial ring in $2 n$ variables over a field $k$. Let $\mathfrak{a}=\left(X_{i}^{2}-Y_{i}^{2}, X_{i} Y_{i} \mid 1 \leq i \leq n\right)$ in $R$ and put $A=R / \mathfrak{a}$. Then:

(1) $\mathrm{a}(A)=2 n$ and $\operatorname{dim}_{k} A=4^{n}$.

(2) $\mathcal{X}_{A}=\varnothing$ if $k=\mathbb{R}$. 
Proof. Since $\left\{X_{i}^{2}-Y_{i}^{2}, X_{i} Y_{i}\right\}_{1 \leq i \leq n}$ form a regular sequence in $R$, the Hilbert series of $A$ is given by

$$
\mathrm{H}(A, \lambda)=\frac{\left(1-\lambda^{2}\right)^{2 n}}{(1-\lambda)^{2 n}}=(1+\lambda)^{2 n},
$$

from which assertion (1) follows. To see assertion (2), let $\Omega=\{1,2, \ldots, n\}$ and $\mathcal{F}=\{I \mid I \subseteq \Omega\}$. Let $x_{i}$ and $y_{i}$ denote respectively the reduction of $X_{i}$ and $Y_{i}$ $\bmod \mathfrak{a}$. Hence $x_{i} y_{i}=0$, so that $x_{i}^{3}=y_{i}^{3}=0$ for all $1 \leq i \leq n$. For each $I \in \mathcal{F}$ we put $x_{I}=\prod_{i \in I} x_{i}$ and $y_{I}=\prod_{i \in I} y_{i}$. We denote by $|I|$ the number of elements in $I$. Then $A_{2 n}=k z$, where $z=x_{\Omega}^{2}=y_{\Omega}^{2}$. The $k$-space $A_{n}$ has the basis $\left\{x_{I} y_{J}^{2} y_{K}\right\}$, where $I, J, K$ run over the elements of $\mathcal{F}$, satisfying the following two conditions:

(1) each two of $I, J, K$ are disjoint, and

(2) $|I|+2|J|+|K|=n$.

Let $\rho: A \stackrel{\pi}{\rightarrow} A_{a} \stackrel{\sigma}{\rightarrow} k$, where $\pi$ denotes the projection map and $\sigma$ the $k$-isomorphism given by $\sigma(z)=1$. We look at the pairing $\langle\rangle:, A_{n} \times A_{n} \rightarrow k,(x, y) \mapsto \rho(x y)$. Let $I, J, K, I^{\prime}, J^{\prime}, K^{\prime} \in \mathcal{F}$ and assume the triples $(I, J, K)$ and $\left(I^{\prime}, J^{\prime}, K^{\prime}\right)$ satisfy the above conditions (1) and (2). Then if $J \neq \varnothing$, we have

$$
\left(x_{I} y_{J}^{2} y_{K}\right)\left(x_{I^{\prime}} y_{J^{\prime}}^{2} y_{K^{\prime}}\right)= \begin{cases}z & \text { if } I=I^{\prime}, K=K^{\prime}, \text { and } J^{\prime}=\Omega \backslash(I \cup J \cup K) ; \\ 0 & \text { otherwise. }\end{cases}
$$

If $J=\varnothing$, we have

$$
\left(x_{I} y_{K}\right)\left(x_{I^{\prime}} y_{J^{\prime}}^{2} y_{K^{\prime}}\right)= \begin{cases}z & \text { if } I=I^{\prime}, K=K^{\prime}, \text { and } J^{\prime}=\varnothing \\ 0 & \text { otherwise. }\end{cases}
$$

Hence the matrix $\mathcal{S}$ of the pairing $\langle\rangle:, A_{n} \times A_{n} \rightarrow k$ with respect to this basis $\left\{x_{I} y_{J}^{2} y_{K}\right\}$ of $A_{n}$ has the form

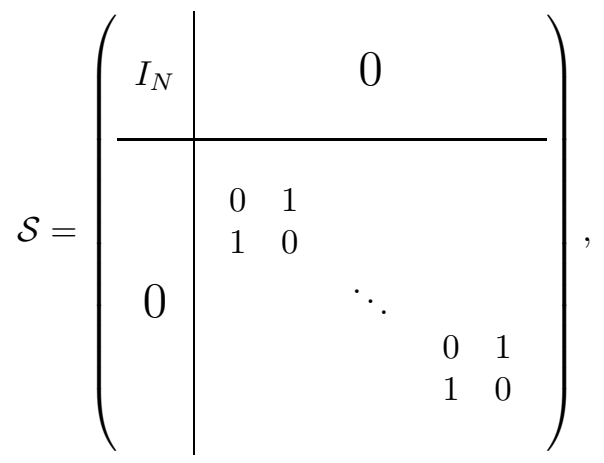

where $N=2^{n}$. Consequently, by (3.5), $\mathcal{X}_{A}=\varnothing$ if $k=\mathbb{R}$.

\section{A Correspondence theorem When $\operatorname{dim} A=1$}

In this section let us assume that $\operatorname{dim} A=1$. Let $K=\mathrm{Q}(A)$ be the total quotient ring of $A$. We denote by $\mathcal{Y}_{A}$ the set of Gorenstein $A$-subalgebras $C$ of $K$ such that $C \supsetneq A$ but the $A$-module $C$ is finitely generated. The main purpose is to establish a one-to-one correspondence between the sets $\mathcal{X}_{A}$ and $\mathcal{Y}_{A}$.

Let $I$ be an $\mathfrak{m}$-primary ideal in $A$. Assume that $I$ contains an element $a$ (necessarily not a zero divisor in $A$ ) such that $I^{n+1}=a I^{n}$ for some $n \geq 0$. To begin with we note 
Lemma (4.1) (cf. [L2], Lemma 1.11). Suppose that $I^{2}=a I$ and let $C=\frac{I}{a}:=$ $\left\{\frac{i}{a} \mid i \in I\right\}$ in $K$. Then:

(1) $C$ is an A-subalgebra of $K$, and $I=a C$.

(2) $C=A$ if and only if $I=a A$.

(3) $A:_{K} C=a A: I$.

(4) $C$ is a Gorenstein ring if $I=a A: I$.

(5) $C=I:_{K} I$.

Proof. As $I^{2}=a I$, we get that $C$ is a subring of $K$ and $A \subseteq C$. We have $C \cong I$ as $A$-modules, since $I=a C$. Therefore the extension $C / A$ is module-finite. Because the element $a$ is invertible in $K$, we get

$$
A:_{K} C=a A:_{K} a C=a A: I .
$$

Hence $C=A$ if and only if $I=a A$. If $I=a A: I$, we have $A:{ }_{K} C \cong C$ as $C$-modules, so that $C$ is Gorenstein (cf. [HK, Satz 5.9 and 5.12]; notice that $\operatorname{Hom}_{A}(C, A) \cong A:_{K} C$.). Assertion (5) is clear, since $I:_{K} I=a C:_{K} a C=$ $C:{ }_{K} C=C$.

Let $I \in \mathcal{X}_{A}$. Then, since $I^{2}=a I$ and $I=a A: I$ for some $a \in I$, by (4.1) we get the Gorenstein $A$-subalgebra $C=\frac{I}{a}$ of $K$. As $a A \neq I$, then $C \neq A$, whence $C \in \mathcal{Y}_{A}$. Because $C=I: K I$ by (4.1) (5), the map $\varphi: \mathcal{X}_{A} \rightarrow \mathcal{Y}_{A}, I \mapsto C$ is well-defined (that is, independent of the choice of the elements $a \in I$ ) and it is an injection, since $A:_{K} C=I((4.1)(3))$. We furthermore have the following.

Theorem (4.2). The map $\varphi: \mathcal{X}_{A} \rightarrow \mathcal{Y}_{A}$ is a bijection which reverses the inclusion, and $\ell_{A}(C / A)=\ell_{A}\left(A / A:_{K} C\right)$ for all $C \in \mathcal{Y}_{A}$.

Proof. Let $C \in \mathcal{Y}_{A}$ and put $I=A:_{K} C$. Then $I \neq A$, as $C \neq A$, so that $\sqrt{I}=\mathfrak{m}$. Since $C$ is a Gorenstein ring, the $C$-module $\operatorname{Hom}_{A}(C, A)$ is by [HK, Satz 5.9 and 5.12] projective and of constant rank 1 , whence $A:_{K} C \cong \operatorname{Hom}_{A}(C, A) \cong C$. Thus $I=a C$ for some $a \in I$, whence $I^{2}=a I$. Therefore $C=\frac{I}{a}$, and so by (4.1) (3) $I=a A: I$. Thus $I \in \mathcal{X}_{A}$ and $C=\varphi(I)$. Hence the map $\varphi: \mathcal{X}_{A} \rightarrow \mathcal{Y}_{A}$ is bijective. As $\ell_{A}(C / A)=\ell_{A}(a C / a A)=\ell_{A}(I / a A)=\ell_{A}(A / I)$ (cf. (2.2)), we get $\ell_{A}(C / A)=\ell_{A}\left(A / A:_{K} C\right)$ for all $C \in \mathcal{Y}_{A}$. Notice that $C=A:_{K}\left(A:_{K} C\right)$ for each $C \in \mathcal{Y}_{A}$ (cf. [BH] 3.3.10]), because $A$ is a Gorenstein local ring and $C$ is a maximal Cohen-Macaulay $A$-module. So, letting $C, D \in \mathcal{X}_{A}$, we have $C \supseteq D$ if and only if $A:_{K} C \subseteq A:_{K} D$, which completes the proof of Theorem (4.2).

Let us summarize some consequences of Lemma (4.1) and Theorem (4.2). First we note

Corollary (4.3). Let $I, J \in \mathcal{X}_{A}$. Then $I=J$ if $I \cong J$ as A-modules.

Proof. Choose a unit $\alpha \in K$ so that the isomorphism $f: I \rightarrow J$ is given by $f(x)=\alpha x$ for $x \in I$. Then since $J=\alpha I$, we have $J:_{K} J=\alpha I:_{K} \alpha I=I:_{K} I$. Hence $I=J$ by (4.1).

Corollary (4.4). Suppose that A has finite CM-representation type; that is, A possesses only finitely many isomorphism classes of indecomposable maximal CohenMacaulay modules. Then $\mathcal{X}_{A}$ is a finite set.

Proof. Let $\mathrm{e}(A)$ denote the multiplicity of $A$. Then $\mu_{A}(I) \leq \mathrm{e}(A)$ for all $I \in \mathcal{X}_{A}$ ([S2, Ch.3, Theorem 1.1]). Hence by (4.3) the set $\mathcal{X}_{A}$ is finite. 
Corollary (4.5). Let $I$ be an $\mathfrak{m}$-primary ideal in $A$ and assume $I^{2}=a I$ for some $a \in A$. Let $\ell \geq 1$ be an integer. Then $\ell=1$ if $I^{\ell} \in \mathcal{X}_{A}$.

Proof. Suppose $\ell \geq 2$. We have $\left(I^{\ell}\right)^{2}=a^{\ell} I^{\ell}$. Let $C=\frac{I^{\ell}}{a^{\ell}}$. Then since $I^{\ell} \in \mathcal{X}_{A}$, by (4.2) $C \in \mathcal{Y}_{A}$, while $C=\frac{I}{a}$ since $I^{\ell}=a^{\ell-1} I$. Therefore $I \subseteq A:_{K} C$ since $I=a C$, which is impossible because $A:_{K} C=I^{\ell}$ by (4.1).

Corollary (4.6). Let $I$ be an $\mathfrak{m}$-primary ideal in $A$, and assume $I^{2}=a I$ for some $a \in A$. Suppose $\mu_{A}(I) \geq 2$, and let $C=\frac{I}{a}$. Then $C$ is a Gorenstein ring if and only if $a A: I \in \mathcal{X}_{A}$.

Proof. We have $I=a C$ and $a A: I=A:_{K} C$ by (4.1). Hence $C \neq A$. Therefore if $C$ is a Gorenstein ring, then $C \in \mathcal{Y}_{A}$, whence $a A: I=A:_{K} C \in \mathcal{X}_{A}$ (cf. the proof of (4.2)). Conversely, suppose that $a A: I \in \mathcal{X}_{A}$, and choose $D \in \mathcal{Y}_{A}$ so that $A:_{K} D=a A: I=A:_{K} C$. Then, because $C$ and $D$ are maximal Cohen-Macaulay $A$-modules, we get

$$
D=A:_{K}\left(A:_{K} D\right)=A:_{K}\left(A:_{K} C\right)=C,
$$

whence $C$ is a Gorenstein ring.

Even though $a A: I \in \mathcal{X}_{A}$, the ideal $I$ is not necessarily good. We give a simple example.

Example (4.7). Let $k[[X, Y]]$ be the formal power series ring over a field $k$ and let $A=k[[X, Y]] /(X Y)$. Let $a \in \mathfrak{m}^{2}$ be a nonzerodivisor in $A$ and let $I=a A: \mathfrak{m}$. Then:

(1) $a A: I \in \mathcal{X}_{A}$, but $I \notin \mathcal{X}_{A}$.

(2) $\mu_{A}(I)=2$ and $I^{2}=a I$.

Proof. Let $\bar{A}$ denote the normalization of $A$. Then $\ell_{A}(\bar{A})=1$. Hence $\mathcal{Y}_{A}=\{\bar{A}\}$ and $\mathcal{X}_{A}=\{\mathfrak{m}\}$, so that $a A: I=\mathfrak{m} \in \mathcal{X}_{A}$. We have $I=(a, z) A$ for some $z \in A \backslash a A$, since $A / a A$ is Gorenstein. Therefore $I \neq \mathfrak{m}$, because $\mu_{A}(\mathfrak{m} / a A)=2$ (note that $\left.a \in \mathfrak{m}^{2}\right)$, and so we get $I \notin \mathcal{X}_{A}$. If $\mu_{A}(I)=1$, then $I=z A$ and $a=\alpha z$ for some $\alpha \in \mathfrak{m}$. Let $x \in \mathfrak{m}$ and write $x z=a y$ with $y \in A$. Then, since $a x=\alpha(x z)=a(\alpha y)$, we have $x=\alpha y$, so that $\mathfrak{m}=\alpha A$, which is impossible. Hence $\mu_{A}(I)=2$. To see that $I^{2}=a I$, it suffices to show that $z^{2} \in a I$. As $z \in \mathfrak{m}$, we have $z^{2}=a \beta$ for some $\beta \in A$. Let $x \in \mathfrak{m}$ and write $x z=a y$ with $y \in A$. Then $y \in \mathfrak{m}$ because $a \notin z A$, whence $y z \in a A$. Therefore, because $x z^{2}=a(x \beta)=a(y z)$, we see $x \beta=y z \in a A$. Hence $\beta \in a A: \mathfrak{m}=I$, and thus $z^{2} \in a I$.

However, we have the following characterization of ideals $I$ for which $a A: I \in$ $\mathcal{X}_{A}$.

Theorem (4.8). Let $I$ be an $\mathfrak{m}$-primary ideal in $A$ with $\mu_{A}(I) \geq 2$. Let $a \in A$ and assume that $I^{n+1}=a I^{n}$ for some $n \geq 0$. Let $R=\mathrm{R}(I)$. Then the following conditions are equivalent.

(1) $\mathrm{K}_{R} \cong R_{+}$as graded $R$-modules.

(2) $I^{2}=a I$ and $a A: I \in \mathcal{X}_{A}$.

Proof. We put $\mathcal{P}=\mathrm{R}(a A)$, the Rees algebra of the ideal $a A$, and let $f=a t \in R_{1}$. Then $f$ is transcendental over $A$, and $\mathcal{P}=A[f]$. Let $\bar{R}=R / f R$. 
(1) $\Rightarrow(2)$ Let $\mathfrak{M}=\mathfrak{m} R+R_{+}$and apply the functors $\mathrm{H}_{\mathfrak{M}}^{i}(*)$ to the exact sequence $0 \rightarrow R(-1) \stackrel{f}{\rightarrow} R \rightarrow \bar{R} \rightarrow 0$ of graded $R$-modules. We get the embedding

$$
0 \rightarrow \mathrm{H}_{\mathfrak{M}}^{0}(\bar{R}) \rightarrow \mathrm{H}_{\mathfrak{M}}^{1}(R)(-1)
$$

of local cohomology modules. On the other hand, we have $\mathrm{H}_{\mathfrak{M}}^{1}\left(R_{+}\right)=\mathrm{H}_{\mathfrak{M}}^{1}\left(\mathrm{~K}_{R}\right)=$ $(0), \operatorname{since} \operatorname{depth}_{R_{\mathfrak{M}}}\left(\mathrm{K}_{R}\right)_{\mathfrak{M}}=2$. Therefore by the exact sequence $0 \rightarrow R_{+} \rightarrow R \stackrel{p}{\rightarrow}$ $A \rightarrow 0$, where $p$ denotes the projection, we get the embedding

$$
0 \rightarrow \mathrm{H}_{\mathfrak{M}}^{1}(R) \rightarrow \mathrm{H}_{\mathfrak{m}}^{1}(A) .
$$

Thus $\mathrm{H}_{\mathfrak{M}}^{1}(R)=\left[\mathrm{H}_{\mathfrak{M}}^{1}(R)\right]_{0}$, so that by $(4.9)$ we get $\mathrm{H}_{\mathfrak{M}}^{0}(\bar{R})=\left[\mathrm{H}_{\mathfrak{M}}^{0}(\bar{R})\right]_{1}$. Now let $r=\mathrm{r}_{a A}(I)(\geq 1)$ and notice that $\mathrm{H}_{\mathfrak{M}}^{0}(\bar{R})=I / a A \oplus \cdots \oplus I^{r} / a I^{r-1}$ (GH, Lemma 2.2]) because $\bar{R}=A \oplus I / a A \oplus \cdots \oplus I^{r} / a I^{r-1}$. Hence $r=1$ and $I^{2}=a I$, since $\mathrm{H}_{\mathfrak{M}}^{0}(\bar{R})$ is concentrated in degree 1 . We put $C=\frac{I}{a}$. Then $C$ is a subring of $K$ and a module-finite extension of $A$. Let $S=\mathrm{R}_{C}(I)$ denote the Rees algebra of $I=a C$, which is considered to be an ideal of $C$. Then $S=C[f]$, and $S$ is the polynomial ring over $C$. We look at the natural exact sequence

$$
0 \rightarrow R \rightarrow S \rightarrow S / R \rightarrow 0
$$

of graded $R$-modules, and note that $S / R=[S / R]_{0} \cong C / A$. Then we have $\mathrm{K}_{R}=$ $R_{+}=S_{+} \cong S(-1)$ and $\ell_{A}(S / R)<\infty$. Hence $\operatorname{Ext}_{\mathcal{P}}^{i}\left(S / R, \mathrm{~K}_{\mathcal{P}}\right)=(0)$ for $i \leq 1$ ( $\mathrm{BH}$ 3.3.10]), so that, taking the $\mathrm{K}_{\mathcal{P}}$-dual of (4.10), we get the isomorphisms

$$
\mathrm{K}_{S}:=\operatorname{Hom}_{\mathcal{P}}\left(S, \mathrm{~K}_{\mathcal{P}}\right) \cong \operatorname{Hom}_{\mathcal{P}}\left(R, \mathrm{~K}_{\mathcal{P}}\right)=\mathrm{K}_{R} \cong S(-1)
$$

by $[\mathrm{BH}]$ 3.3.7]. Therefore by [HK, Satz 5.12$] S=C[f]$ is a Gorenstein ring because $\mathrm{K}_{S} \cong S(-1)$, whence so is $C$ because $C$ is a polynomial ring over $C$. Thus by (4.6) $a A: I \in \mathcal{X}_{A}$.

$(2) \Rightarrow(1)$ Let $C=\frac{I}{a}$. Then $C \in \mathcal{Y}_{A}$ by (4.6), and $I=a C$. Let $S=\mathrm{R}_{C}(I)$. Then $S=C[f]$ is the polynomial ring, so that $S$ is a Gorenstein ring with $\mathrm{K}_{S}=S(-1)$. Therefore, since $\mathrm{K}_{R}=\operatorname{Hom}_{\mathcal{P}}\left(R, \mathrm{~K}_{\mathcal{P}}\right) \cong \mathrm{K}_{S}=\operatorname{Hom}_{\mathcal{P}}\left(S, \mathrm{~K}_{\mathcal{P}}\right)$ by (4.10), we have $\mathrm{K}_{R} \cong \mathrm{K}_{S} \cong S(-1) \cong S_{+}$. Thus $\mathrm{K}_{R} \cong R_{+}$, since by (4.10) $R_{+} \cong S_{+}$. This completes the proof of Theorem (4.8).

As an immediate consequence we have

Corollary (4.11). Suppose that $I \in \mathcal{X}_{A}$ and let $R=\mathrm{R}(I)$. Then $\mathrm{K}_{R}=R_{+}$.

We now discuss the question of when $\mathcal{X}_{A}=\varnothing$. To do this we need the following fact.

Lemma (4.12). Let $I$ be an $\mathfrak{m}$-primary ideal in $A$ with $\mu_{A}(I) \geq 2$. Let $a \in I$ be such that $I^{n+1}=a I^{n}$ for some $n \geq 0$. Let $r=\mathrm{r}_{a A}(I)$. Then $I^{r} \in \mathcal{X}_{A}$ if $\mathrm{G}(I)$ is a Gorenstein ring.

Proof. Let $G=\mathrm{G}(I)$ and $R^{\prime}=\mathrm{R}^{\prime}(I)$. Then $R^{\prime}$ is a Gorenstein ring with $\mathrm{K}_{R^{\prime}} \cong$ $R^{\prime}(r)$, since $G$ is a Gorenstein ring with a $(G)=r-1$ (see the proof of $(2.2)$ ). Let $R^{\prime(r)}=\sum_{i \in \mathbb{Z}} R_{i r}^{\prime}$ denote the Veronesean subring of $R^{\prime}$ with order $r$, and identify $\mathrm{R}^{\prime}\left(I^{r}\right)=R^{\prime(r)}$. Then we have

$$
\mathrm{K}_{\mathrm{R}^{\prime}\left(I^{r}\right)}=\mathrm{K}_{R^{\prime}(r)}=\left(\mathrm{K}_{R^{\prime}}\right)^{(r)} \cong\left[R^{\prime}(r)\right]^{(r)}=\left[{R^{\prime}}^{(r)}\right](1)=\left[\mathrm{R}^{\prime}\left(I^{r}\right)\right](1) .
$$


Therefore, since $R^{\prime(r)}$ is Cohen-Macaulay (cf. [HE, Proposition 12]), it is a Gorenstein ring with $\mathrm{K}_{\mathrm{R}^{\prime}\left(I^{r}\right)} \cong\left[\mathrm{R}^{\prime}\left(I^{r}\right)\right](1)$. Hence by $(2.2) I^{r}$ is a good ideal in $A$.

Lemma (4.13). Let $I$ be an $\mathfrak{m}$-primary ideal in $A$ with $\mu_{A}(I) \geq 2$. Let $a \in I$ be such that $I^{n+1}=a I^{n}$ for some $n \geq 0$. Let $r=\mathrm{r}_{a A}(I)$. Then $a^{r} A: I^{r} \in \mathcal{X}_{A}$ if Proj $\mathrm{R}(I)$ is a Gorenstein scheme.

Proof. Let $R=\mathrm{R}(I)$. Then since $\sqrt{R_{+}}=\sqrt{(a t)}$, we have Proj $\mathrm{R}(I)=\operatorname{Spec} C$ with $C=A\left[\frac{i}{a} \mid i \in I\right]=I^{r} / a^{r}$. Therefore $C$ is a Gorenstein ring such that $I^{r}=a^{r} C$, and so $\left(I^{r}\right)^{2}=a^{r} I^{r}$. We claim that $\mu_{A}\left(I^{r}\right) \geq 2$. In fact, suppose that $\mu_{A}\left(I^{r}\right)=1$. Then $I^{r}=a^{r} A$ (cf. [S2, Ch.2, Theorem 1.7]). Let $x \in I$. Then, because $a^{r-1} x \in I^{r}$, we get $a^{r-1} x \in a^{r} A$, whence $x \in a A$. Thus $I=a A$, which is impossible because $\mu_{A}(I) \geq 2$ by our assumption. Hence by (4.6) $a^{r} A: I^{r}$ is a good ideal in $A$.

Proposition (4.14). Suppose that $A / \mathfrak{m}$ is infinite. Then the following conditions are equivalent.

(1) $\mathcal{X}_{A}=\varnothing$.

(2) Every $\mathfrak{m}$-primary ideal I in A with a Gorenstein associated graded ring $\mathrm{G}(I)$ is principal.

(3) Every $\mathfrak{m}$-primary ideal I in A for which Proj $\mathrm{R}(I)$ is a Gorenstein scheme is principal.

Proof. Since the field $A / \mathfrak{m}$ is infinite, every $\mathfrak{m}$-primary ideal $I$ in $A$ contains an element $a$ such that $I^{n+1}=a I^{n}$ (cf. [NR] or [S2, Ch.2, Theorem 2.2]).

$(1) \Rightarrow(2)$ and $(1) \Rightarrow(3)$ See (4.12) and (4.13).

$(2) \Rightarrow(1)$ This is clear, since no good ideal is principal.

$(3) \Rightarrow(1)$ Assume that $\mathcal{X}_{A} \neq \varnothing$ and let $I \in \mathcal{X}_{A}$. Choose $a \in I$ so that $I^{2}=a I$. Then Proj $\mathrm{R}(I)=\operatorname{Spec} C$ with $C=\frac{I}{a}$. Hence Proj $\mathrm{R}(I)$ is a Gorenstein scheme, so that the ideal $I$ has to be principal. This is absurd.

To close this section we note the following.

Proposition (4.15). The following conditions are equivalent.

(1) $A$ is a discrete valuation ring.

(2) $\hat{A}$ is reduced and $\mathcal{X}_{A}=\varnothing$.

Proof. (1) $\Rightarrow(2)$ This is clear.

$(2) \Rightarrow(1)$ Assume the contrary and let $\bar{A}$ denote the normalization of $A$. Then, since $\bar{A}$ is certainly a Gorenstein $\operatorname{ring}$ and $\bar{A} \neq A$, we have $\bar{A} \in \mathcal{Y}_{A}$ so that by (4.2) $\mathcal{X}_{A} \neq \varnothing$, which is impossible.

Question (4.16). Is $\widehat{A}$ a reduced ring if $\mathcal{X}_{A}=\varnothing$ ?

We suspect that $A$ is a discrete valuation ring, if $\mathcal{X}_{A}=\varnothing$.

\section{Good ideals in OnE-Dimensional REDUCED COMPLETE LOCAL RINGS OF MULTIPLICITY 2}

The purpose of this section is to prove the following.

Theorem (5.1). Let $A$ be a one-dimensional reduced complete local ring with $\mathrm{e}_{\mathfrak{m}}(A)=2$, and let $B=\bar{A}$ denote the normalization of $A$. Then:

(1) Every intermediate ring $A \subseteq C \subseteq B$ is a Gorenstein ring. 
(2) $\sharp \mathcal{X}_{A}=\ell_{A}(B / A):=n$.

(3) The set $\mathcal{X}_{A}$ is totally ordered with respect to inclusion.

Hence there is a unique chain $A=C_{0} \subseteq C_{1} \subseteq \cdots \subseteq C_{n}=B$ of rings between $A$ and $B$.

We divide the proof of Theorem (5.1) into several steps. For a moment let $(A, \mathfrak{m})$ be a Noetherian local $\operatorname{ring}$ with $\operatorname{dim} A=1$. Let $\mathrm{e}(A)=\mathrm{e}_{\mathfrak{m}}(A)$ denote the multiplicity of $A$ with respect to the maximal ideal $\mathfrak{m}$ and let $\mathrm{v}(A)=\ell_{A}\left(\mathfrak{m} / \mathfrak{m}^{2}\right)$ denote the embedding dimension of $A$. Then we have the following lemma, which is known, but we give a brief proof for completeness.

Lemma (5.2). Assume that $\widehat{A}$ is a reduced ring and let $\bar{A}$ denote the normalization of $A$. Then $\mathrm{e}(A)=\mu_{A}(\bar{A})$ and $\mathrm{v}(A) \leq \mathrm{e}(A)$.

Proof. See $[1$ for the inequality $\mathrm{v}(A) \leq \mathrm{e}(A)$. Since $\bar{A}$ is a principal ideal ring, we may choose $t \in \bar{A}$ so that $\mathfrak{m} \bar{A}=t \bar{A}$. Then $t$ is a nonzerodivisor in $\bar{A}$, and we have $\mathfrak{m}^{i} \bar{A} / \mathfrak{m}^{i+1} \bar{A} \cong \bar{A} / \mathfrak{m} \bar{A}$. Hence $\ell_{A}\left(\mathfrak{m}^{i} \bar{A} / \mathfrak{m}^{i+1} \bar{A}\right)=\ell_{A}(\bar{A} / \mathfrak{m} \bar{A})$ for all $i \geq 0$, so that $\mathrm{e}(A)=\ell_{A}(\bar{A} / \mathfrak{m} \bar{A})=\mu_{A}(\bar{A})$ because $\mathrm{e}(A)=\mathrm{e}_{\mathfrak{m}}^{0}(\bar{A})$.

Now let us assume that $A$ is a reduced complete local $\operatorname{ring}$ with $\operatorname{dim} A=1$ and $\mathrm{e}(A)=2$. Let $K=\mathrm{Q}(A)$ denote the total quotient ring of $A$ and let $B$ denote the normalization of $A$. Then by (5.2) we have $\mathrm{v}(A) \leq 2$. Hence $A$ is a hypersurface with $\sharp$ Ass $A \leq 2$, and the ring $A$ is an integral domain if $\sharp$ Ass $A=1$. We first discuss the case where $\sharp$ Ass $A=2$. So, take a complete regular local ring $(R, \mathfrak{n})$ with $\operatorname{dim} R=2$ and let $P_{1}, P_{2}$ be distinct prime ideals in $R$ such that $R / P_{i}$ is a discrete valuation $\operatorname{ring}(i=1,2)$. Let us identify $A=R / P_{1} \cap P_{2}$. We write $P_{1}=(X)$ and $P_{2}=(Z)$ with $X, Z \in \mathfrak{n}$, and choose $Y \in \mathfrak{n}$ so that $\mathfrak{n}=(X, Y)$. Then, because $Y \bmod P_{1}$ is a regular parameter of $R / P_{1}$, we have $Z=a X+\varepsilon Y^{n}$ for some $a \in R, \varepsilon \in R^{\times}$, and $n \geq 1$. Note that $a \in R^{\times}$if $n \geq 2$, because $Z \notin \mathfrak{n}^{2}$. Let $x, y$, and $z$ denote respectively the reduction of $X, Y$, and $Z \bmod P_{1} \cap P_{2}$. Then we have

Proposition (5.3). (1) $\sharp \mathcal{X}_{A}=\ell_{A}(B / A)=n$.

(2) $\mathcal{X}_{A}=\left\{\left(x, y^{i}\right) \mid 1 \leq i \leq n\right\}$.

(3) Every intermediate ring $A \subseteq C \subseteq B$ is a Gorenstein ring.

Proof. We put $\mathfrak{p}_{1}=(x)$ and $\mathfrak{p}_{2}=(z)$. Then, since

$$
B=A / \mathfrak{p}_{1} \times A / \mathfrak{p}_{2} \text { and } A /\left(\mathfrak{p}_{1}+\mathfrak{p}_{2}\right)=R /\left(X, Y^{n}\right),
$$

we see that $\ell_{A}(B / A)=n$ and $A:_{K} B=\left(x, y^{n}\right)$. Hence $\left(x, y^{n}\right) \in \mathcal{X}_{A}$. Let $I \in \mathcal{X}_{A}$. Then by (4.2) $\left(x, y^{n}\right) \subseteq I$, and so $I=\left(x, y^{i}\right)$ for some $1 \leq i \leq n$, because $A /(x)$ is a discrete valuation ring with $y \bmod (x)$ a regular parameter. We want to show, conversely, that $\left(x, y^{i}\right) \in \mathcal{X}_{A}$ for all $1 \leq i \leq n$. We may assume $n>1$. Let $1 \leq i \leq n$ and put $I=\left(x, y^{i}\right)$. Then $I^{2}=y^{i} I+\left(x^{2}\right)$. As $Z=a X+\varepsilon Y^{n}$, we have $0=x z=a x^{2}+\varepsilon x y^{n}$. Since $a \in R^{\times}$, we see that $x^{2} \in\left(x y^{n}\right) \subseteq y^{i} I$, whence $I^{2}=y^{i} I$. We particularly have $\mathfrak{m}^{2}=y \mathfrak{m}$, since $I=\mathfrak{m}$ for $i=1$. Therefore

$$
\ell_{A}\left(A / y^{i} A\right)=i \cdot \ell_{A}(A / y A)=i \cdot \mathrm{e}(A)=2 i,
$$

while

$$
\ell_{A}(A / I)=\ell_{R}\left(R /\left(X, Y^{i}\right)=i\right.
$$


Thus by (2.2) $I \in \mathcal{X}_{A}$, so that we have $\mathcal{X}_{A}=\left\{\left(x, y^{i}\right) \mid 1 \leq i \leq n\right\}$. Hence $\sharp \mathcal{X}_{A}=n=\ell_{A}(B / A)$. To see assertion (3), let $A \subseteq C \subseteq B$ be any intermediate ring. Then, since $A$ is complete and $C$ is a finitely generated $A$-module, the ring $C$ is a finite direct product of local rings, say $C=C_{1} \times C_{2} \times \cdots \times C_{\ell}$. We have $\ell \leq 2$, because $\sharp$ Ass $C=\sharp$ Ass $K=\sharp$ Ass $A=2$. If $\ell=1$, then $C$ is a local ring with $\mu_{C}(B) \leq \mu_{A}(B)=2$. Hence $\mathrm{e}(C) \leq 2$ by (5.2), so that $C$ is a Gorenstein ring. Suppose $\ell=2$ and choose $t \in \mathfrak{m}$ so that $\mathfrak{m}^{2}=t \mathfrak{m}$ (this choice is possible even in the case where $n=1$; take $t=x-z)$. Then $\mathrm{e}(A)=\mathrm{e}_{t A}^{0}(A)=\mathrm{e}_{t A}^{0}(C)=$ $\mathrm{e}_{t A}^{0}\left(C_{1}\right)+\mathrm{e}_{t A}^{0}\left(C_{2}\right)=\ell_{A}\left(C_{1} / t C_{1}\right)+\ell_{A}\left(C_{2} / t C_{2}\right)$. Therefore for $i=1,2$ we have $\ell_{A}\left(C_{i} / t C_{i}\right)=1$, so that $C_{i}$ is a discrete valuation ring, whence $C=B$. Thus any intermediate ring $A \subseteq C \subseteq B$ is a Gorenstein ring.

We now assume that $A$ is an integral domain. Then $B$ is a discrete valuation ring. Let $A \subseteq C \subseteq B$ be any intermediate ring. Then $C$ is a local ring, as so is $B$. We denote by $\kappa(C)$ the residue class field of $C$. Since e $(C)=\mu_{C}(B) \leq \mu_{A}(B)=2$ by (5.2), $C$ is a Gorenstein local ring. Let $\mathfrak{n}$ denote the maximal ideal in $B$ and let $s=[\kappa(B): \kappa(A)]$. Then $s \leq 2$, since $s \leq \mu_{A}(B)=2$. First we consider the case $s=1$ and choose $t \in \mathfrak{n}$ so that $\mathfrak{n}=t B$. Then $\mathfrak{m} B=t^{2} B$ and $B=A+t A$. We write $A:_{K} B=t^{\ell} B$ with $\ell \geq 1$. Then $\ell=2 \ell_{A}(B / A)$, because $\ell_{A}(B / A)=$ $\frac{1}{2} \ell_{A}\left(B / t^{\ell} B\right)$ (cf. (4.2)) and $\ell_{A}\left(B / t^{\ell} B\right)=\ell_{B}\left(B / t^{\ell} B\right)=\ell$. Let $n=\ell_{A}(B / A)$, and put $C_{i}=A+t^{2 i-1} B$ for each $1 \leq i \leq n$. Then the $C_{i}$ 's are subrings of $B$ with $C_{1}=B, C_{n} \neq A$ (recall that $t^{2 n-1} B \nsubseteq A$ ). We furthermore have

Proposition (5.4). $\mathcal{Y}_{A}=\left\{C_{i} \mid 1 \leq i \leq n\right\}$ and $\sharp \mathcal{Y}_{A}=n$.

Proof. We may assume that $n>1$ and that the assertion is true for $n-1$. Notice that $C_{n}=A+t^{2 n-1} A$, since $B=A+t A$ and $t^{2 n} B \subseteq A$. Besides, since $\mathfrak{m} \subseteq t^{2} B$ we have $\mathfrak{m} t^{2 n-1} \subseteq t^{2 n+1} B \subseteq A$. Hence $\ell_{A}\left(C_{n} / A\right)=1<n$. Therefore $B \supsetneq C_{n}$, so that $\mathrm{e}\left(C_{n}\right)=2$. Since $\ell_{A}\left(B / C_{n}\right)=n-1$ and $\left[\kappa\left(C_{n}\right): \kappa(A)\right]=1$, we get $\ell_{C_{n}}\left(B / C_{n}\right)=n-1$. Because $\left[\kappa(B): \kappa\left(C_{n}\right)\right]=1$, by the hypothesis of induction on $n$ we have that $\sharp \mathcal{Y}_{C_{n}}=n-1$ and that $\mathcal{Y}_{C_{n}}=\left\{D_{i} \mid 1 \leq i \leq n-1\right\}$, where $D_{i}=C_{n}+t^{2 i-1} B=\left(A+t^{2 n-1} B\right)+t^{2 i-1} B=C_{i}$ for all $1 \leq i \leq n-1$; hence $\mathcal{Y}_{C_{n}}=\left\{C_{i} \mid 1 \leq i \leq n-1\right\}$. Now let $C \in \mathcal{Y}_{A}$ and write $C:_{K} B=t^{i} B$ with $i \geq 0$. Then, because $[\kappa(B): \kappa(C)]=[\kappa(C): \kappa(A)]=1$ and $\ell_{C}(B / C)=\ell_{C}\left(C / C:_{K} B\right)$ by (4.2) (cf. [HK, Korollar 3.7] also), we have $\ell_{A}(B / C)=\ell_{C}(B / C)=\frac{1}{2} \ell_{C}\left(B / t^{i} B\right)=$ $\frac{1}{2} \ell_{B}\left(B / t^{i} B\right)=\frac{i}{2}$, so that $n>\frac{i}{2}$ as $A \subsetneq C$. Therefore $C \supseteq t^{i} B \supseteq t^{2 n-1} B$, and so $C \supseteq C_{n}$. Thus either $C=C_{n}$ or $C \in \mathcal{Y}_{C_{n}}$, whence $\mathcal{Y}_{A}=\mathcal{Y}_{C_{n}} \cup\left\{C_{n}\right\}=\left\{C_{i} \mid 1 \leq\right.$ $i \leq n\}$, so that we have $\sharp \mathcal{Y}_{C_{n}}=n$.

Finally, we consider the case $[\kappa(B): \kappa(A)]=2$. Since $\mathfrak{n}=\mathfrak{m} B$, we have $\mathfrak{n}=t B$ for some $t \in \mathfrak{m}$. Choose $\theta \in B$ so that $B=A+\theta A$. Let $A:_{K} B=t^{n} B$ with $n \geq 1$. Then $n=\ell_{A}(B / A)$, since $\ell_{A}(B / A)=\frac{1}{2} \ell_{A}\left(B / t^{n} B\right)$ by (4.2) and $\ell_{A}\left(B / t^{n} B\right)=2 \ell_{B}\left(B / t^{n} B\right)=2 n$. Let $C_{i}=A+t^{i} B(0 \leq i \leq n-1)$. Then we have the following.

Proposition (5.5). $\mathcal{Y}_{A}=\left\{C_{i} \mid 0 \leq i \leq n-1\right\}$ and $\sharp \mathcal{Y}_{A}=n$.

Proof. We may assume that $n>1$ and that the assertion is true for $n-1$. Because $t \in A$ and $B=A+\theta A$, we have $C_{n-1}=A+\theta t^{n-1} A$. Hence $\ell_{A}\left(C_{n-1} / A\right)=1<n=$ $\ell_{A}(B / A)$, because $\mathfrak{m} \theta t^{n-1} \subseteq t^{n} B \subseteq A$, so that $B \supsetneq C_{n-1}$ and $\mathrm{e}\left(C_{n-1}\right)=2$. Because the maximal ideal $\mathfrak{M}$ of $C_{n-1}$ contains the element $t$, we have $\mathfrak{M} B=\mathfrak{n}$, whence $\left[\kappa(B): \kappa\left(C_{n-1}\right)\right]=2$ and $\left[\kappa\left(C_{n-1}\right): \kappa(A)\right]=1$, as $B \neq C_{n-1}$. Consequently 
$\ell_{C_{n-1}}\left(B / C_{n-1}\right)=\ell_{A}\left(B / C_{n-1}\right)=n-1$. Thus by the hypothesis of induction on $n$ we have $\sharp \mathcal{Y}_{C_{n-1}}=n-1$ and $\mathcal{Y}_{C_{n-1}}=\left\{D_{i} \mid 0 \leq i \leq n-2\right\}$, where $D_{i}=C_{n-1}+t^{i} B=$ $\left(A+t^{n-1} B\right)+t^{i} B=C_{i}$ for $0 \leq i \leq n-2$; hence $\mathcal{Y}_{C_{n-1}}=\left\{C_{i} \mid 0 \leq i \leq n-2\right\}$. Let $C \in \mathcal{Y}_{A}$ and let $C:_{K} B=t^{i} B$ with $i \geq 0$. We want to show that $C \supseteq C_{n-1}$. To do this we may assume $C \subsetneq B$. Hence $[\kappa(B): \kappa(C)]=2$, so that $[\kappa(C): \kappa(A)]=1$. Therefore $n>\ell_{A}(B / C)=\ell_{C}(B / C)=\ell_{C}\left(C / C:_{K} B\right)=i$. Hence $C \supseteq t^{n-1} B$, so that $C \supseteq C_{n-1}$. Thus $\mathcal{Y}_{A}=\mathcal{Y}_{C_{n-1}} \cup\left\{C_{n-1}\right\}$, which completes the proof of Theorem (5.1) as well as that of Proposition (5.5).

Example (5.6). Typical examples of one-dimensional reduced complete local rings $A$ with $\mathrm{e}(A)=2$ are as follows. Let $k$ be a field and $n \geq 1$ an integer.

(I) $(\sharp$ Ass $A=2)$ Let $A=k[[X, Y]] /(X) \cap\left(X+Y^{n}\right)$, where $k[[X, Y]]$ denotes the formal power series ring in two variables $X, Y$ over $k$.

(II) ( $A$ is an integral domain with $[\kappa(B): \kappa(A)]=1)$ Let $A=k\left[\left[t^{2}, t^{2 n+1}\right]\right] \subseteq$ $k[[t]]$, where $k[[t]]$ denotes the formal power series ring in one variable over $k$. Then $B=k[[t]]$.

(III) ( $A$ is an integral domain with $[\kappa(B): \kappa(A)]=2$ ) Let $K / k$ be an extension of fields with $[K: k]=2$, and choose $\theta \in K$ so that $K=k+k \theta$. Let $A=k\left[\left[t, \theta t^{n}\right]\right] \subseteq K[[t]]$, where $K[[t]]$ denotes the formal power series ring in one variable over $K$. Then $B=K[[t]]$.

For these rings $A$ we always have $\sharp \mathcal{X}_{A}=n$.

Even though $\mathrm{e}(A)=2$, the set $\mathcal{X}_{A}$ is not necessarily finite unless $\widehat{A}$ is reduced, as we shall show in the following. Let $(R, \mathfrak{n})$ be a discrete valuation ring with $t$ a regular parameter. Let $F$ denote the quotient field of $R$. Let $A=R[X] /\left(X^{2}\right)$ and $K=F[X] /\left(X^{2}\right)$, where $X$ denotes an indeterminate over $F$. Then $K$ is the total quotient ring of $A$. Let $x$ denote the reduction of $X \bmod \left(X^{2}\right)$ and let $B$ be the integral closure of $A$ in $K$. Then we have the following.

Example (5.7). (1) $\mathcal{X}_{A}=\left\{\left(t^{i}, x\right) \mid i \geq 1\right\}$. Hence the set $\mathcal{X}_{A}$ is infinite, which is a totally ordered set with respect to inclusion.

(2) Every module-finite extension of $A$ in $K$ is a Gorenstein ring. Hence between $A$ and $B$ there is a unique chain

$$
A=C_{0} \subsetneq C_{1} \subsetneq \cdots \subsetneq C_{i}=R\left[\frac{x}{t^{i}}\right] \subsetneq \cdots \subsetneq B
$$

of rings, consisting of module-finite extensions of $A$.

Proof. Since $A=R+R x$ and $K=F+F x$, we have $B=R+F x$. For each $i \geq 1$ we have $\ell_{A}\left(A /\left(t^{i}, x\right)\right)=i$ and $\ell_{A}\left(A / t^{i} A\right)=2 i$. Therefore by $(2.2)\left(t^{i}, x\right) \in \mathcal{X}_{A}$, since $\left(t^{i}, x\right)^{2}=t^{i}\left(t^{i}, x\right)$. Let $A \subseteq C \subseteq B$ be an intermediate ring and assume that $C$ is a module-finite extension of $A$. Then $C$ is a local ring, as $B$ is. Let $\mathfrak{m}_{C}$ be the maximal ideal in $C$. Then $\mathfrak{m}_{C}=t R+(C \cap F x)$, so that $R / t R=C / \mathfrak{m}_{C}$. Therefore, as $\mathfrak{m}_{C}{ }^{2}=t \cdot \mathfrak{m}_{C}$, we get $\mathrm{e}(C)=2$ since $\ell_{R}(C / t C)=2$ (notice that $C$ is a free $R$-module of rank 2). Hence $C$ is a Gorenstein ring. Choose $p \in \mathbb{Z}$ so that $C=R+R t^{p} x$ (this choice is possible, since $C=R+(C \cap F x)$ and $C \cap F x$ is a free $R$-module of rank 1). Then we have $p<0$, as $C \supsetneq A$, whence $C=R\left[x / t^{i}\right]$ with $i=-p>0$, so that $A:_{K} C=\left(t^{i}, x\right)$.

Let $k$ be a field and $B=k[[t]]$ the formal power series ring over $k$. Let $K$ denote the quotient field of $B$. The next example shows that $\sharp \mathcal{X}_{A}$ depends on the ground field. 
Example (5.8). Let $A=k\left[\left[t^{4}, t^{5}, t^{6}\right]\right]$. Then $\mathcal{X}_{A}=\left\{\left(t^{8}, t^{9}, t^{10}, t^{11}\right),\left(t^{6}, t^{8}, t^{9}\right)\right\} \cup$ $\left\{\left(t^{4}-\lambda t^{5}, t^{6}-\lambda^{2} t^{8}\right) \mid \lambda \in k\right\}$ and $\mathcal{Y}_{A}=\left\{B, k\left[\left[t^{2}, t^{3}\right]\right]\right\} \cup\left\{A\left[t^{2}+\lambda t^{3}\right] \mid \lambda \in k\right\}$. Hence $\sharp \mathcal{X}_{A}=\sharp k+2$, and so the set $\mathcal{X}_{A}$ is infinite if $k$ is.

Proof. Let $\theta_{\lambda}=t^{2}+\lambda t^{3}$ and $C_{\lambda}=A\left[\theta_{\lambda}\right]$ for each $\lambda \in k$. Then we have $C_{\lambda}=$ $A+A \theta_{\lambda}=k+k \theta_{\lambda}+t^{4} B$. Let $\mathfrak{m}_{\lambda}$ denote the maximal ideal in $C_{\lambda}$. Then $\mathfrak{m}_{\lambda}=$ $\left(\theta_{\lambda}, t^{4}, t^{5}, t^{6}\right) C_{\lambda}$, and so $\mathfrak{m}_{\lambda} B \subseteq \theta_{\lambda} B=t^{2} B$. Hence $\mathfrak{m}_{\lambda} \subseteq \theta_{\lambda} B \cap C_{\lambda}=\overline{\theta_{\lambda} C_{\lambda}}$ (here $\bar{*}$ denotes the integral closure of ideals), whence $\theta_{\lambda} C_{\lambda}$ is a reduction of $\mathfrak{m}_{\lambda}$. Therefore $\mathrm{e}\left(C_{\lambda}\right)=\ell_{C_{\lambda}}\left(B / \theta_{\lambda} B\right)=2$. Thus $C_{\lambda}$ is a Gorenstein ring and $C_{\lambda} \in \mathcal{Y}_{A}$. Let $\lambda, \mu \in k$ and assume that $C_{\lambda}=C_{\mu}$. Then, since $\theta_{\lambda} \in C_{\mu}=k+k \theta_{\mu}+t^{4} B$, we have $t^{2}+\lambda t^{3}=a+b\left(t^{2}+\mu t^{3}\right)+t^{4} f$ for some $a, b \in k$ and $f \in B$. Then $a=0, b=1$, and $\lambda=b \mu$, whence $\lambda=\mu$. As $t^{3} \notin C_{\lambda}$ for any $\lambda \in k$, we find that $C_{\lambda} \neq B, k\left[\left[t^{2}, t^{3}\right]\right]$. Thus $B, k\left[\left[t^{2}, t^{3}\right]\right]$, and $\left\{C_{\lambda}\right\}_{\lambda \in k}$ form a distinct subfamily of $\mathcal{Y}_{A}$. We have

$$
\begin{gathered}
A:_{K} B=t^{8} B=\left(t^{8}, t^{9}, t^{10}, t^{11}\right) A, \\
A:_{K} k\left[\left[t^{2}, t^{3}\right]\right]=t^{6} k\left[\left[t^{2}, t^{3}\right]\right]=\left(t^{6}, t^{8}, t^{9}\right) A,
\end{gathered}
$$

and

$$
A:{ }_{K} C_{\lambda}=\left(t^{4}-\lambda t^{5}\right) C_{\lambda}=\left(t^{4}-\lambda t^{5}, t^{6}-\lambda^{2} t^{8}\right) A .
$$

Hence $\mathcal{X}_{A} \ni\left(t^{8}, t^{9}, t^{10}, t^{11}\right),\left(t^{6}, t^{8}, t^{9}\right)$, and $\left(t^{4}-\lambda t^{5}, t^{6}-\lambda^{2} t^{8}\right)$ for all $\lambda \in k$.

Conversely, let $C \in \mathcal{Y}_{A}$ and write $C:_{K} B=t^{2 \ell} B$ with $\ell=\ell_{A}(B / C)$. Here we note that $t^{7} \in C$. In fact, suppose $t^{7} \notin C$. Then $C:_{K} B=t^{8} B$, since $A:_{K} B=t^{8} B \subseteq A \subseteq C$. Hence $\ell_{A}(B / C)=\ell_{A}(B / A)=4$, so that we have $C=A$, which is impossible. Thus $t^{7} \in C$ and so $t^{4} B \subseteq C$, since $t^{4}, t^{5}, t^{6} \in A$ and $t^{8} B \subseteq A$. Hence $\ell \leq 2$. We have $C=B$ if $\ell=0$, and $C=k\left[\left[t^{2}, t^{3}\right]\right]$ if $\ell=1$. Let $\ell=2$, and let $\mathfrak{m}_{C}$ be the maximal ideal in $C$. Then $\mathfrak{m}_{C} \supseteq t^{4} B$ and $\ell_{c}\left(C / t^{4} B\right)=2$, whence $\mathfrak{m}_{C}=k \theta+t^{4} B$ for some $\theta \in \mathfrak{m}_{C}$ with $\theta \notin t^{4} B$. We have $\theta=a t^{2}+b t^{3}$ with $a, b \in k$, as $C \neq B$. If $a=0$, then $\mathfrak{m}_{C}=k t^{3}+t^{4} B=t^{3} B$, so that $C=k\left[\left[t^{3}, t^{4}, t^{5}\right]\right]$, which is impossible because $k\left[\left[t^{3}, t^{4}, t^{5}\right]\right]$ is not a Gorenstein ring. Hence $a \neq 0$ and $\mathfrak{m}_{C}=k \theta_{\lambda}+t^{4} B$ for some $\lambda \in k$. Thus $C=k+k \theta_{\lambda}+t^{4} B_{\lambda}=C_{\lambda}$.

\section{A characterization of good ideals When $\operatorname{dim} A=2$}

Let $A$ be a Gorenstein local ring with the maximal ideal $\mathfrak{m}$ and $\operatorname{dim} A=2$. Let $I$ be an $\mathfrak{m}$-primary ideal in $A$ and assume that $I$ contains a parameter ideal $Q=(a, b)$ in $A$ as a reduction. Then for any integer $n \geq 1$ the ideal $\left(a^{n}, b^{n}\right)$ is a reduction of $I^{n}$. Let $r=\mathrm{r}_{Q}(I)$. We put $G=\mathrm{G}(I), R=\mathrm{R}(I)$, and $R^{\prime}=\mathrm{R}^{\prime}(I)$. Let $\mathfrak{M}=\mathfrak{m} G+G_{+}$.

The purpose of this section is to prove the following theorem, in which the equivalence of conditions (1), (6), and (9) is already given by (2.2).

Theorem (6.1). The following nine conditions are equivalent to each other.

(1) $I \in \mathcal{X}_{A}$.

(2) $I^{\ell} \in \mathcal{X}_{A}$ for all $\ell \geq 1$.

(3) $I^{2}=Q I$, and $I^{\ell} \in \mathcal{X}_{A}$ for some $\ell \geq 1$.

(4) The Hilbert function $\ell_{A}\left(A / I^{n+1}\right)$ is a polynomial in $n$ for all $n \geq 0$, and $I^{\ell} \in \mathcal{X}_{A}$ for some $\ell \geq 1$.

(5) $\mathrm{H}_{\mathfrak{M}}^{1}(G)=(0)$, and $I^{\ell} \in \mathcal{X}_{A}$ for some $\ell \geq 1$.

(6) $I^{n}=Q^{n}: I$ for all $n \in \mathbb{Z}$.

(7) $\ell_{A}\left(A / I^{n}\right)=n^{2} \ell_{A}(A / I)$ for all $n \geq 1$. 
(8) $R$ is a Cohen-Macaulay ring, and $\mathrm{K}_{R} \cong R_{+}$as graded $R$-modules.

(9) $R^{\prime}$ is a Gorenstein ring, and $\mathrm{K}_{R^{\prime}} \cong R^{\prime}$ as graded $R^{\prime}$-modules.

When this is the case, the equality

$$
\mu_{A}\left(I^{n}\right)=n \cdot \mu_{A}(I)-n+1
$$

holds true for all $n \geq 1$.

We divide the proof of Theorem (6.1) into several steps. To begin with we give

Lemma (6.2). The following conditions are equivalent.

(1) $I \in \mathcal{X}_{A}$.

(2) $I^{\ell} \in \mathcal{X}_{A}$ for all $\ell \geq 1$

(3) $\ell_{A}\left(A / I^{n}\right)=n^{2} \ell_{A}(A / I)$ for all $n \geq 1$.

When this is the case, we have $\mu_{A}\left(I^{n}\right)=n \cdot \mu_{A}(I)-n+1$ for all $n \geq 1$.

Proof. Let $I \in \mathcal{X}_{A}$ and let $n \geq 1$ be an integer. Then by (2.2) $R^{\prime}$ is a Gorenstein ring, and $\mathrm{K}_{R^{\prime}} \cong R^{\prime}$ as graded $R^{\prime}$-modules. Hence, looking at the Veronesean subring of $R^{\prime}$ with order $n$, we see that $\mathrm{R}^{\prime}\left(I^{n}\right)$ is a Gorenstein ring with $\mathrm{K}_{\mathrm{R}^{\prime}\left(I^{n}\right)}=\mathrm{R}^{\prime}\left(I^{n}\right)$ (see the proof of Lemma (4.12)). Thus by (2.2) $I^{n} \in \mathcal{X}_{A}$, whence $\ell_{A}\left(A / I^{n}\right)=\frac{1}{2} \cdot \ell_{A}\left(A /\left(a^{n}, b^{n}\right)\right)=\frac{1}{2} \cdot n^{2} \ell_{A}(A / Q)$. Hence $\ell_{A}\left(A / I^{n}\right)=n^{2} \ell_{A}(A / I)$, as $\ell_{A}(A / Q)=2 \ell_{A}(A / I)$. Conversely assume condition (3) is satisfied. Then, because $\ell_{A}\left(A / I^{n+1}\right)=(n+1)^{2} \ell_{A}(A / I)=2 \ell_{A}(A / I)\left(\begin{array}{c}n+2 \\ 2\end{array}\right)-\ell_{A}(A / I)\left(\begin{array}{c}n+1 \\ 1\end{array}\right)$ for all $n \geq 0$, thanks to a theorem of Huneke [H] we get $I^{2}=Q I$ and $\ell_{A}(A / I)=\frac{1}{2} \mathrm{e}_{I}(A)=$ $\frac{1}{2} \ell_{A}(A / Q)$. Hence $I \in \mathcal{X}_{A}$. The last assertion is now clear, since $I^{2}=Q I$ (cf. Sh].

Lemma (6.3). The following conditions are equivalent.

(1) $I \in \mathcal{X}_{A}$.

(2) $R$ is a Cohen-Macaulay ring, and $\mathrm{K}_{R} \cong R_{+}$as graded $R$-modules.

Proof. (1) $\Rightarrow(2)$ Since $G$ is Cohen-Macaulay and a $(G)<0$, by GS (1.1) and (3.10)] the ring $R$ is Cohen-Macaulay. We put $\mathcal{P}=\mathrm{R}(Q)=A[a t, b t]$. Then $\mathcal{P}$ is a Gorenstein ring with $\mathrm{a}(\mathcal{P})=-1$, because $\mathcal{P} \cong A[X, Y] /(a Y-b X)$ (here $A[X, Y]$ is the polynomial ring in two variables $X, Y$ over $A$ ). Therefore $\mathrm{K}_{\mathcal{P}} \cong \mathcal{P}(-1)$, so that we have $\mathrm{K}_{R} \cong \operatorname{Hom}_{\mathcal{P}}(R, \mathcal{P}(-1)) \cong[\mathcal{P}: R](-1)$, where $\mathcal{P}: R$ denotes the conductor. Hence the isomorphism $\mathrm{K}_{R} \cong R_{+}$follows modulo the following.

Claim. $\mathcal{P}: R=I R \cong R_{+}(1)$.

Proof of Claim. We have $I R=I \mathcal{P}$, as $I^{n+1}=I Q^{n}$ for all $n \geq 0$, whence $I R \subseteq$ $\mathcal{P}: R$. Let $c \in Q^{n}$ with $n \geq 0$, and assume that $c t^{n} \in \mathcal{P}: R$. Then $c x \in Q^{n+1}$ for all $x \in I$, since $c t^{n} \cdot x t \in \mathcal{P}$. Therefore $c \in Q^{n+1}: I=I^{n+1}$ by (2.2), so that $c t^{n} \in I \mathcal{P}$. Thus $\mathcal{P}: R=I R \cong R_{+}(1)$.

$(2) \Rightarrow(1)$ The ring $G$ is Cohen-Macaulay, as $R$ is $([\mathrm{GS}])$. Since $\mathrm{K}_{R}=R_{+} \cong$ $[I R](-1)$, we have the canonical exact sequence $0 \rightarrow\left[\mathrm{K}_{R}\right](1) \rightarrow R \rightarrow G \rightarrow 0$ of graded $R$-modules. Apply functors $\mathrm{H}_{\mathfrak{N}}^{i}(*)$ to it, where $\mathfrak{N}=\mathfrak{m} R+R_{+}$, and we have the exact sequence

$$
0 \rightarrow \mathrm{H}_{\mathfrak{M}}^{2}(G) \rightarrow \mathrm{H}_{\mathfrak{N}}^{3}\left(\mathrm{~K}_{R}\right)(1)
$$

of local cohomology modules. Because $\mathrm{H}_{\mathfrak{N}}^{3}\left(\mathrm{~K}_{R}\right)=\mathrm{E}_{R}(R / \mathfrak{N})$ is the injective envelope of $R / \mathfrak{N}$, the socle $(0):_{\mathrm{H}_{\mathfrak{N}}^{3}}\left(\mathrm{~K}_{R}\right) \mathfrak{N}$ has length 1 and is concentrated in degree 0 . 
Therefore, thanks to the above embedding, we see that the socle $(0):_{\mathrm{H}_{\mathfrak{M}}^{2}}(G) \mathfrak{M}$ of $\mathrm{H}_{\mathfrak{M}}^{2}(G)$ has length 1 and is concentrated in degree -1 , too. Thus $G$ is a Gorenstein ring with a $(G)=-1$, whence $I \in \mathcal{X}_{A}$ by definition.

Thanks to lemmas (6.2) and (6.3), we have done with the equivalence of conditions (1), (2), (6), (7), (8), and (9) in Theorem (6.1). To check the other equivalences, we need the following.

Theorem (6.4). Let $N \geq 0$ be an integer and suppose that the equality

$$
\ell_{A}\left(A / I^{n+1}\right)=\mathrm{e}_{I}^{0}(A)\left(\begin{array}{c}
n+2 \\
2
\end{array}\right)-\mathrm{e}_{I}^{1}(A)\left(\begin{array}{c}
n+1 \\
1
\end{array}\right)+\mathrm{e}_{I}^{2}(A)
$$

holds true for all integers $n \geq N$. Assume that $I^{\ell} \in \mathcal{X}_{A}$ for some $\ell \geq 1$. Then the following assertions hold true.

(1) $\mathrm{e}_{I}^{2}(A)=0$ and $\mathrm{e}_{I}^{1}(A)=\frac{1}{2} \mathrm{e}_{I}^{0}(A)$.

(2) $\ell_{A}\left(A /\left(I^{k}\right)^{n+1}\right)=(n+1)^{2} \ell_{A}\left(A / I^{k}\right)$ for all integers $k \geq N+1$ and $n \geq 0$.

(3) $I^{k} \in \mathcal{X}_{A}$ if $k \geq N+1$.

(4) $I \in \mathcal{X}_{A}$ if and only if $\mathrm{H}_{\mathfrak{M}}^{1}(G)=(0)$.

Proof. We put $N_{1}=\max \left\{0, \frac{N-\ell+1}{\ell}\right\}$ and choose $n \geq N_{1}$. Then, since $\ell n+\ell-1 \geq N$, we have

$$
\begin{aligned}
\ell_{A}\left(A /\left(I^{\ell}\right)^{n+1}\right) & =\ell_{A}\left(A / I^{\ell n+\ell}\right) \\
& =\mathrm{e}_{I}^{0}(A)\left(\begin{array}{c}
\ell n+\ell+1 \\
2
\end{array}\right)-\mathrm{e}_{I}^{1}(A)\left(\begin{array}{c}
\ell n+\ell \\
1
\end{array}\right)+\mathrm{e}_{I}^{2}(A),
\end{aligned}
$$

while by (6.2) we get

$$
\begin{aligned}
\ell_{A}\left(A /\left(I^{\ell}\right)^{n+1}\right) & =(n+1)^{2} \ell_{A}\left(A / I^{\ell}\right) \\
& =2 \ell_{A}\left(A / I^{\ell}\right)\left(\begin{array}{c}
n+2 \\
2
\end{array}\right)-\ell_{A}\left(A / I^{\ell}\right)\left(\begin{array}{c}
n+1 \\
1
\end{array}\right),
\end{aligned}
$$

because $I^{\ell} \in \mathcal{X}_{A}$. Hence, comparing the above two equalities (6.5) and (6.6), we get $\mathrm{e}_{I}^{2}(A)=0$ and $\mathrm{e}_{I}^{1}(A)=\ell_{A}\left(A / I^{\ell}\right)=\mathrm{e}_{I}^{0}(A) / 2$. Let $k \geq N+1$ and $n \geq 0$. Then $k n+k-1 \geq N$, and so we have

$$
\begin{aligned}
\ell_{A}\left(A /\left(I^{k}\right)^{n+1}\right) & =\ell_{A}\left(A / I^{k n+k}\right) \\
& =\mathrm{e}_{I}^{0}(A)\left(\begin{array}{c}
k n+k+1 \\
2
\end{array}\right)-\frac{\mathrm{e}_{I}^{0}(A)}{2}\left(\begin{array}{c}
k n+k \\
1
\end{array}\right) \\
& =\frac{\mathrm{e}_{I}^{0}(A)}{2} k^{2}(n+1)^{2} .
\end{aligned}
$$

Since $\ell_{A}\left(A / I^{k}\right)=\frac{1}{2} \mathrm{e}_{I}^{0}(A) k^{2}$ (take $n=0$ in $\left.(6.7)\right)$, we have

$$
\ell_{A}\left(A /\left(I^{k}\right)^{n+1}\right)=(n+1)^{2} \ell_{A}\left(A / I^{k}\right)
$$

for all $n \geq 0$, whence by (6.2) $I^{k} \in \mathcal{X}_{A}$ for all $k \geq N+1$. Therefore by $(2.2) \mathrm{R}^{\prime}\left(I^{k}\right)$ is a Gorenstein ring with $\mathrm{K}_{\mathrm{R}^{\prime}\left(I^{k}\right)} \cong \mathrm{R}^{\prime}\left(I^{k}\right)$. Hence, identifying $\mathrm{R}^{\prime}\left(I^{k}\right)=R^{\prime(k)}$, we see that $\left[\mathrm{K}_{R^{\prime}}\right]^{(k)} \cong R^{\prime(k)}$ as graded $R^{\prime(k)}$-modules. Thus $\left[\mathrm{K}_{R^{\prime}}\right]_{0} \cong A$. We now choose $\theta \in\left[\mathrm{K}_{R^{\prime}}\right]_{0}$ so that $\left[\mathrm{K}_{R^{\prime}}\right]_{0}=A \theta$, and we let $\varphi: R^{\prime} \rightarrow \mathrm{K}_{R^{\prime}}$ denote the homomorphism of graded $R^{\prime}$-modules defined by $\varphi(1)=\theta$. We put $\mathcal{K}=\operatorname{Ker} \varphi$ and $\mathcal{W}=\operatorname{Coker} \varphi$. Then, since $\left[\mathrm{K}_{R^{\prime}}\right]^{(k)}={R^{\prime}}^{(k)} \theta$, we have $\mathcal{K}_{k}=(0)$ and $\mathcal{W}_{k}=(0)$ for all $k \geq N+1$. 
Hence $\mathcal{K}$ and $\mathcal{W}$ are finitely graded $R^{\prime}$-modules. Consequently, because at $\in R^{\prime}$ is a nonzerodivisor of $R^{\prime}$ and $a t^{n} \cdot \mathcal{K}=(0)$ for all $n \gg 0$, we get $\mathcal{K}=(0)$ and have the exact sequence

$$
0 \rightarrow R^{\prime} \stackrel{\varphi}{\rightarrow} \mathrm{K}_{R^{\prime}} \rightarrow \mathcal{W} \rightarrow 0
$$

of graded $R^{\prime}$-modules.

Claim. $\ell_{A}(\mathcal{W})<\infty$.

Proof of Claim. Since $\mathcal{W}$ is a finitely generated $A$-module, it suffices to show that $\mathcal{W}_{\mathfrak{p}}=(0)$ for all $\mathfrak{p} \in \operatorname{Spec} A \backslash\{\mathfrak{m}\}$. Because $R_{\mathfrak{p}}^{\prime}=A_{\mathfrak{p}}\left[t, t^{-1}\right]$ is a Gorenstein ring and $\left[\mathrm{K}_{R^{\prime}}\right]_{\mathfrak{p}}=\mathrm{K}_{R_{\mathfrak{p}}^{\prime}}$, we have that $\left[\mathrm{K}_{R^{\prime}}\right]_{\mathfrak{p}}$ is generated by the element $\frac{\theta}{1}$ as an $R_{\mathfrak{p}^{-}}^{\prime}$ module. Hence $A_{\mathfrak{p}} \otimes_{A} \varphi$ is an isomorphism, so that we have $\mathcal{W}_{\mathfrak{p}}=(0)$. Hence $\ell_{A}(\mathcal{W})<\infty$.

We now look at the exact sequence

$$
0 \rightarrow\left[(0): \mathcal{W} t^{-1}\right](1) \rightarrow G \rightarrow \mathrm{K}_{R^{\prime}} / t^{-1} \mathrm{~K}_{R^{\prime}} \rightarrow \mathcal{W} / t^{-1} \mathcal{W} \rightarrow 0
$$

of graded $G$-modules induced from (6.8). Then, since $\ell_{A}(\mathcal{W})<\infty$, applying the functors $\mathrm{H}_{\mathfrak{M}}^{i}(*)$ to it, we have the isomorphism

$$
\left[(0): \mathcal{W} t^{-1}\right](1) \cong \mathrm{H}_{\mathfrak{M}}^{0}(G)
$$

and the embedding

$$
0 \rightarrow \mathcal{W} / t^{-1} \mathcal{W} \rightarrow \mathrm{H}_{\mathfrak{M}}^{1}(G)
$$

of local cohomology modules (recall that $\mathrm{H}_{\mathfrak{M}}^{0}\left(\mathrm{~K}_{R^{\prime}} / t^{-1} \mathrm{~K}_{R^{\prime}}\right)=(0)$, because the canonical modules always have depth at least 2 if the dimension of the base ring is at least 2). Therefore if $\mathrm{H}_{\mathfrak{M}}^{1}(G)=(0)$, then thanks to Nakayama's lemma we get $\mathcal{W}=(0)$ by $(6.10)$, so that $\mathrm{H}_{\mathfrak{M}}^{0}(G)=(0)$ by $(6.9)$. Thus $G$ is a Cohen-Macaulay ring, whence so is $R^{\prime}$. The ring $R^{\prime}$ is actually a Gorenstein ring, because the homomorphism $\varphi: R^{\prime} \rightarrow \mathrm{K}_{R^{\prime}}$ is an isomorphism. Hence by (2.2) $I \in \mathcal{X}_{A}$, which completes the proof of Theorem (6.4).

We are now in a position to finish the proof of Theorem (6.1). The implications $(1) \Rightarrow(3) \Rightarrow(5)$ are now clear, and the implication $(1) \Rightarrow(4)$ follows from (6.2). We have the implications $(4) \Rightarrow(1)$ and $(5) \Rightarrow(1)$ by Theorem (6.4).

Let us give some consequences of Theorems (6.1) and (6.4).

Corollary (6.11). Suppose that $A$ is a two-dimensional rational local ring. Then $I \in \mathcal{X}_{A}$ if and only if $\bar{I}=I$ and $I^{\ell} \in \mathcal{X}_{A}$ for some $\ell \geq 1$.

Proof. If $\bar{I}=I$, then $I^{2}=Q I$ by $[\mathrm{LT}$. Hence the assertion follows from (2.5) and (6.1).

Even though $I^{\ell} \in \mathcal{X}_{A}$ for all $\ell \gg 0$, the ideal $I$ is not necessarily a good ideal in $A$. Let us give one example.

Example (6.12). Let $k[[X, Y, Z]]$ be the formal power series ring over a field $k$ and let $A=k[[X, Y, Z]] /\left(Z^{2}-X Y\right)$. Let $x, y$, and $z$ denote respectively the reduction of $X, Y$, and $Z \bmod \left(Z^{2}-X Y\right)$. We put $I=\left(x^{2}, y^{2}, x z, y z\right)$. Then $I^{\ell} \in \mathcal{X}_{A}$ for all $\ell \geq 2$, but $I \notin \mathcal{X}_{A}$. 
Proof. Since $\mathrm{G}(\mathfrak{m}) \cong k[X, Y, Z] /\left(Z^{2}-X Y\right), \mathrm{G}(\mathfrak{m})$ is a Gorenstein ring with a $(\mathrm{G}(\mathfrak{m}))$ $=-1$ (GW, 3.1.6]). Hence $\mathfrak{m} \in \mathcal{X}_{A}$, and so by $(6.2) \mathfrak{m}^{\ell} \in \mathcal{X}_{A}$ for all $\ell \geq 1$. As $\mathfrak{m}^{2}=$ $(x, y) \mathfrak{m}$, the elements $x^{2}, y^{2}$ generates a reduction of $\mathfrak{m}^{2}$, whence $\mathfrak{m}^{4}=\left(x^{2}, y^{2}\right) \mathfrak{m}^{2}$. Consequently, because the ideal $\left(x^{2}, y^{2}\right) \subseteq I \subseteq \mathfrak{m}^{2},\left(x^{2}, y^{2}\right)$ is a reduction of $I$ too. Hence if $I \in \mathcal{X}_{A}$, then by (2.4) $I=\mathfrak{m}^{2}$, so that we have $\mu_{A}\left(\mathfrak{m}^{2}\right) \leq 4$, while $\mu_{A}\left(\mathfrak{m}^{2}\right)=2 \mu_{A}(\mathfrak{m})-2+1=5$ by (6.2). Thus $I \notin \mathcal{X}_{A}$. It is standard to check that $I^{2}=\mathfrak{m}^{4}$. Hence $I^{\ell}=\mathfrak{m}^{2 \ell}$ for all $\ell \geq 2$. In fact, suppose that $\ell \geq 3$ and $I^{\ell-1}=\mathfrak{m}^{2 \ell-2}$. Then, because $I^{\ell}=I I^{\ell-1}=I \mathfrak{m}^{2 \ell-2}=\left(I \mathfrak{m}^{2}\right) \mathfrak{m}^{2 \ell-4}$ and $I \mathfrak{m}^{2} \supseteq I^{2}=\mathfrak{m}^{4}$, we get $I^{\ell}=\mathfrak{m}^{2 \ell}$. Thus $I^{\ell} \in \mathcal{X}_{A}$ for all $\ell \geq 2$. Since $\mathfrak{m} \in \mathcal{X}_{A}$, we have $\ell_{A}\left(A / I^{n+1}\right)=\ell_{A}\left(A / \mathfrak{m}^{2 n+2}\right)=(2 n+2)^{2}=4(n+1)^{2}$ for all $n \geq 1$, while $\ell_{A}(A / I)=5$.

The following result is due to Huneke and Swanson [HS]. We give a brief proof for completeness.

Proposition (6.13) ([$[\mathrm{HS}]$ ). Suppose that $A$ is a Cohen-Macaulay local ring, and let $I$ be a perfect ideal in $A$ with $\mathrm{ht}_{A} I=2$. Then $(x, y): I \nsubseteq I$ for any part $\{x, y\}$ of a minimal system of generators of $I$.

Proof. Let $0 \rightarrow A^{n-1} \stackrel{\varphi}{\rightarrow} A^{n} \rightarrow I \rightarrow 0$ be a minimal free resolution of the $A$-module $I$, and let $d_{i}=(-1)^{i} \operatorname{det} \varphi_{i}$ for each $1 \leq i \leq n$, where $\varphi_{i}$ denotes the $(n-1) \times(n-1)$ matrix obtained from $\varphi$ by deleting the $i^{\text {th }}$ row. Then, thanks to Hilbert-Burch's theorem, we may assume $d_{1}=x, d_{2}=y$ and $I=\left(d_{1}, d_{2}, \ldots, d_{n}\right)$. Let $\psi$ be the $(n-2) \times(n-1)$ matrix obtained from $\varphi$ by deleting the first two rows, and for each $1 \leq j \leq n-1$ let

$$
\Delta_{j}=(-1)^{j} \operatorname{det} \psi_{j}
$$

where $\psi_{j}$ denotes the $(n-2) \times(n-2)$ matrix obtained from $\psi$ by deleting the $j$ th column. Let $J=\left(\Delta_{1}, \Delta_{2}, \ldots, \Delta_{n-1}\right)$. Then

$$
\varphi\left[\begin{array}{c}
\Delta_{1} \\
\Delta_{2} \\
\Delta_{3} \\
\vdots \\
\Delta_{n-1}
\end{array}\right]=\left[\begin{array}{c}
-d_{2} \\
d_{1} \\
0 \\
\vdots \\
0
\end{array}\right],
$$

so that $J \subseteq(x, y): I$. Now notice that $J \nsubseteq I$, because $d_{1} \in \mathfrak{m} J$ but $d_{1} \notin \mathfrak{m} I$. Finally, we have $(x, y): I \nsubseteq I$ because $J \subseteq(x, y): I$.

The following are immediate consequences of Proposition (6.13). See Proposition (7.5) for an alternative proof of Corollary (6.15).

Corollary (6.14). Let $I \in \mathcal{X}_{A}$. Then $\operatorname{pd}_{A} A / I=\infty$.

Corollary (6.15). Suppose that $A$ is a regular local ring of $\operatorname{dim} A=2$. Then $\mathcal{X}_{A}=\varnothing$.

We don't know whether the converse of Corollary (6.15) is also true.

Question (6.16). Suppose that $\operatorname{dim} A=2$. Then is $A$ a regular local ring if $\mathcal{X}_{A}=\varnothing ?$

Let us add two more examples. 
Example (6.17). Let $(R, \mathfrak{n})$ be a regular local $\operatorname{ring}$ with $\operatorname{dim} R=3$, and assume that $R / \mathfrak{n}$ is infinite. Let $f \in \mathfrak{n}^{2} \backslash \mathfrak{n}^{3}$. Then $\mathfrak{n} / f R \in \mathcal{X}_{R / f R}$, so that $(\mathfrak{n} / f R)^{\ell} \in \mathcal{X}_{R / f R}$ for all $\ell \geq 1$.

Proof. This follows from the isomorphism $\mathrm{G}(\mathfrak{n} / f R) \cong \kappa[X, Y, Z] /(F)$, where $F$ is a form of degree 2 in the polynomial ring $\kappa[X, Y, Z]$ in three variables over $\kappa=R / \mathfrak{n}$.

Example (6.18). Let $R=k[[X, Y, Z]]$ be the formal power series ring over a field $k$, and let $\mathfrak{a}=\left(Z^{c}-X^{a} Y^{b}\right)(a, b \geq 1$ and $c \geq 2)$. We put $A=R / \mathfrak{a}$. Let $x, y$, and $z$ denote respectively the reduction of $X, Y$, and $Z \bmod \mathfrak{a}$. Then:

(1) $I=\left(x^{m}\right)+(y, z)^{c-1} \in \mathcal{X}_{A}$ for all $1 \leq m \leq a$.

(2) If $c$ is even, then $I=\left(x^{m}, y^{n}, z^{\frac{c}{2}}\right) \in \mathcal{X}_{A}$ for all $1 \leq m \leq a$ and $1 \leq n \leq b$.

Proof. (1) As $I=\left(x^{m}, y^{c-1}\right)+z(y, z)^{c-2}$, we have $I^{2}=\left(x^{m}, y^{c-1}\right) I+z^{2}(y, z)^{2 c-4}$. Let $\alpha, \beta \geq 2$ be integers such that $\alpha+\beta=2 c-4$, and look at the form $\xi=$ $z^{2} y^{\alpha} z^{\beta}$. If $\alpha \geq c-1$, then $\xi=y^{c-1}\left(z^{2} y^{\alpha-c+1} z^{\beta}\right)$ with $z^{2} y^{\alpha-c+1} z^{\beta} \in(y, z)^{c-1}$. Suppose $\alpha \leq c-2$. Then $\beta \geq c-2$ and $\xi=z^{c}\left(y^{\alpha} z^{\beta-c+2}\right)=\left(x^{a} y^{b}\right)\left(y^{\alpha} z^{\beta-c+2}\right)=$ $x^{m}\left(x^{a-m} y^{b+\alpha} z^{\beta-c+2}\right)$ with $y^{b+\alpha} z^{\beta-c+2} \in I$, because $(b+\alpha)+(\beta-c+2)=b+$ $c-2 \geq c-1$. Thus $I^{2}=\left(x^{m}, y^{c-1}\right) I$. Hence $I \in \mathcal{X}_{A}$ by $(2.2)$, as $\ell_{A}(A / I)=$ $\ell_{R}\left(R /\left(X^{m}\right)+(Y, Z)^{c-1}\right)=m(c-1) c / 2$ and $\ell_{A}\left(A /\left(x^{m}, y^{c-1}\right)\right)=m(c-1) c$.

(2) Notice that $I^{2}=\left(x^{m}, y^{n}\right) I, \ell_{A}(A / I)=m n c / 2$, and $\ell_{A}\left(A /\left(x^{m}, y^{n}\right)\right)=m n c$.

In the case where the base rings are rational local rings, good ideals are closely related to the theory of adjoints of ideals defined by J. Lipman. Let $A$ be a twodimensional rational local ring and let $I$ be an ideal in $A$. Let $f: X \rightarrow \operatorname{Spec} A$ be a desingularization with $I \mathcal{O}_{X}$ invertible. Let $\widetilde{I}=\mathrm{H}^{0}\left(X, \mathcal{O}_{X}\right)$ and call it the adjoint of $I([\mathrm{~L} 4])$. This definition is independent of the choice of desingularizations $f: X \rightarrow \operatorname{Spec} A$, and we have the following.

Remark (6.19). Let $A$ be a two-dimensional Gorenstein rational excellent local ring. Let $I$ be an m-primary ideal in $A$ with $Q$ a minimal reduction. Then:

(1) $\widetilde{I}=Q: I$ if $\bar{I}=I$.

(2) $I \in \mathcal{X}_{A}$ if and only if $\widetilde{I}=I$.

Proof. (1) This is due to [L4].

(2) By (1) the only if part follows from (2.5). If $\widetilde{I}=I$, then $\bar{I}=I$ (since $\widetilde{I}$ is integrally closed), so that $I=Q: I$ by (1). Thus $I \in \mathcal{X}_{A}$, since $I^{2}=Q I$ ([LT]).

\section{Good ideals in two-dimensional Gorenstein Rational LOCAL RingS}

Let $A$ be a two-dimensional Gorenstein rational local ring with the maximal ideal $\mathfrak{m}$, and assume that the ring $A$ is excellent to assure the existence of desingularizations of $\operatorname{Spec} A$ ([L3] $)$. The purpose of this section is to completely classify good ideals in $A$. Surprisingly, it turns out that there are only countably many good ideals. Let $k[[X, Y, Z]]$ be the formal power series ring over a field $k$. Then the simplest example is the ring

$$
A=k[[X, Y, Z]] /\left(Z^{2}-X Y\right),
$$


in which the good ideals are powers of the maximal ideal $\mathfrak{m}$ in it. In the case where

$$
A=k[[X, Y, Z]] /\left(Z^{3}-X Y\right),
$$

the situation is a little more complicated. The good ideals are products of the following three ideals:

$$
\mathfrak{m}, \quad I_{1}=\left(x, y^{2}, y z, z^{2}\right), \quad I_{2}=\left(x^{2}, x z, y, z^{2}\right),
$$

where $x, y$, and $z$ denote respectively the reduction of $X, Y$, and $Z \bmod \left(Z^{3}-X Y\right)$. Therefore, since $I_{1} I_{2}=\mathfrak{m}^{3}$, the good ideals $I$ in $A$ are written as $I=\mathfrak{m}^{a} I_{1}^{b}$ or $I=\mathfrak{m}^{a} I_{2}^{b}$ for some integers $a, b \geq 0$ with $a+b \geq 1$.

Our method of classification is to look at desingularizations $f: X \rightarrow \operatorname{Spec} A$. By (2.5) every good ideal $I$ in $A$ is integrally closed. Hence, if we take $f: X \rightarrow \operatorname{Spec} A$ to be a desingularization of the blowing-up of $I$, then the invertible sheaf $I \mathcal{O}_{X}$ is isomorphic to $\mathcal{O}_{X}(-Z)$ for some divisor $Z$ on $X$ and $I=\mathrm{H}^{0}\left(X, \mathcal{O}_{X}(-Z)\right)$. The crucial point is that for each $I \in \mathcal{X}_{A}$ we can choose the desingularization $f: X \rightarrow \operatorname{Spec} A$ to be the minimal resolution of $\operatorname{Spec} A$ (Theorem (7.8)).

To enter into details, in what follows, let $A$ denote a two-dimensional excellent normal local ring with the maximal ideal $\mathfrak{m}$. Let $f: X \rightarrow \operatorname{Spec} A$ be a desingularization of $\operatorname{Spec} A$. Let $E=\bigcup_{i=1}^{r} E_{i}$ denote the exceptional divisor on $X$ with the irreducible components $\left\{E_{i}\right\}_{1 \leq i \leq r}$. We begin with the following.

Definition (7.1). Let $I$ be an $\mathfrak{m}$-primary ideal in $A$. Then the ideal $I$ is said to be represented on $X$ if the sheaf $I \mathcal{O}_{X}$ is invertible. In this case we have $I \mathcal{O}_{X}=$ $\mathcal{O}_{X}(-Z)$ for some divisor $Z=\sum_{i=1}^{r} n_{i} E_{i}$ and $I=\mathrm{H}^{0}\left(X, \mathcal{O}_{X}(-Z)\right)$.

First we notice that the colength $\ell_{A}(A / I)$ of $I=\mathrm{H}^{0}\left(X, \mathcal{O}_{X}(-Z)\right)$ is described by Kato's Rieman-Roch formula (7.2). In $\underline{K}$ the theorem corresponding to our proposition (7.2) is stated only for complex manifolds of dimension 2 , but the proof still works in any characteristic. For each invertible sheaf $\mathcal{L}$ on $X$ let

$$
\chi(\mathcal{L})=\ell_{A}\left(\mathrm{H}^{0}(X-E, \mathcal{L}) / \mathrm{H}^{0}(X, \mathcal{L})\right)+\ell_{A}\left(\mathrm{H}^{1}(X, \mathcal{L})\right)
$$

Because $X-E \cong \operatorname{Spec} A \backslash\{\mathfrak{m}\}$, we have that $\mathrm{H}^{0}(X-E, \mathcal{L})$ is the reflexive hull of $\mathrm{H}^{0}(X, \mathcal{L})$, and so $0 \leq \chi(\mathcal{L}) \in \mathbb{Z}$. Let $K_{X}$ denote the canonical divisor of $X$ (see (7.4) below). Then we have the following result, whose proof is not difficult and can be done by the induction on $\sum_{i=1}^{r}\left|n_{i}\right|([\mathrm{G})$.

Proposition (7.2) $([\overline{\mathrm{K}}])$. (1) Let $D=\sum_{i=1}^{r} n_{i} E_{i}$ be a divisor supported on $E$ and put $\mathcal{L}=\mathcal{O}_{X}(D)$. Then

$$
\chi\left(\mathcal{O}_{X}(D)\right)-\chi\left(\mathcal{O}_{X}\right)=\chi\left(\mathcal{O}_{X}(D)\right)-\ell_{A}\left(H^{1}\left(X, \mathcal{O}_{X}\right)\right)=\frac{K_{X} D-D^{2}}{2} .
$$

(2) Let $A$ be a rational local ring and assume that $D=-Z$ for some effective divisor $Z$ on $X$. Let $I=\mathrm{H}^{0}\left(X, \mathcal{O}_{X}(-Z)\right)$. Then

$$
\ell_{A}(A / I)=\frac{-K_{X} Z-Z^{2}}{2} .
$$

Now let us summarize some basic facts on desingularizations.

Fact (7.3) ([L1]). Assume that $A$ is a rational local ring.

(1) The product of two integrally closed $\mathfrak{m}$-primary ideals in $A$ is again integrally closed. 
(2) There is a one-to-one correspondence between the set of integrally closed $\mathfrak{m}$-primary ideals $I$ in $A$ that are represented on $X$ and the set of effective divisors $Z=\sum_{i=1}^{r} n_{i} E_{i}$ on $X$ with $Z E_{i} \leq 0$ for all $1 \leq i \leq r$. The correspondence is given by

$$
I \mathcal{O}_{X}=\mathcal{O}_{X}(-Z) \text { and } I=\mathrm{H}^{0}\left(X, \mathcal{O}_{X}(-Z)\right) .
$$

We call those effective divisors $Z=\sum_{i=1}^{r} n_{i} E_{i}$ with $Z E_{i} \leq 0$ for all $1 \leq i \leq r$ anti-nef.

Fact (7.4). (cf. [La] $)$ Since the intersection matrix $\left[E_{i} E_{j}\right]_{1 \leq i, j \leq r}$ is negative definite, there is a unique $\mathbb{Q}$-divisor $K_{X}=\sum_{i=1}^{r} a_{i} E_{i}\left(a_{i} \in \mathbb{Q}\right)$ on $X$ such that

$$
p_{a}\left(E_{i}\right)=\frac{\left(E_{i}^{2}+K_{X} E_{i}\right)}{2}+\ell_{A}\left(\mathrm{H}^{0}\left(E_{i}, \mathcal{O}_{E_{i}}\right)\right)
$$

for all $1 \leq i \leq r$, where $p_{a}\left(E_{i}\right)$ denotes the arithmetic genus of $E_{i}$. The rational numbers $a_{i}$ are called the discrepancy of the $E_{i}$ 's. If $A$ is a Gorenstein local ring, then $a_{i} \in \mathbb{Z}$ for all $1 \leq i \leq r$, and $\mathcal{O}_{X}\left(K_{X}\right)$ is a canonical sheaf on $X$.

Since $p_{a}\left(E_{i}\right)$ is a non-negative integer and $K_{X} E_{i}+E_{i}^{2} \geq-2$, we see that $K_{X} E_{i} \geq$ 0 except when $K_{X} E_{i}=E_{i}^{2}=-1$ and $p_{a}\left(E_{i}\right)=0$. Hence $-K_{X}$ is anti-nef and $a_{i} \leq 0$ for all $1 \leq i \leq r$, if $f: X \rightarrow \operatorname{Spec} A$ is a minimal resolution (cf. (7.6)). We furthermore have $a_{i}<0$ for all $1 \leq i \leq r$, if $K_{X} \neq 0$. Therefore, because $K_{X}=0$ if and only if $f: X \rightarrow \operatorname{Spec} A$ is a minimal resolution and $A$ is a Gorenstein rational local ring, summarizing these arguments, we get the following assertions.

(1) $A$ is a regular local ring if and only if $a_{i}>0$ for all $1 \leq i \leq r$.

(2) $A$ is a rational local ring if and only if $a_{i} \geq 0$ for all $1 \leq i \leq r$.

(3) If $A$ is a Gorenstein rational singularity, then $a_{i}=0$ if and only if $E_{i}$ appears in the minimal resolution of $\operatorname{Spec} A$.

(4) Suppose that $A$ is not a Gorenstein rational local ring. Then $a_{i}<0$ once $E_{i}$ appears in the minimal resolution of $\operatorname{Spec} A$.

We now start the main argument of this section. The formula (7.2) gives an inequality between the multiplicity and the colength of $\mathfrak{m}$-primary integrally closed ideals. Namely,

Proposition (7.5). The following assertions hold true.

(1) $A$ is a regular local ring if and only if $\ell_{A}(A / I)>\frac{1}{2} \mathrm{e}_{I}^{0}(A)$ for every integrally closed $\mathfrak{m}$-primary ideal $I$ in $A$. (Hence $\mathcal{X}_{A}=\varnothing$ if $A$ is a regular local ring.)

(2) $A$ is a Gorenstein rational local ring if and only if $\ell_{A}(A / I) \geq \frac{1}{2} \mathrm{e}_{I}^{0}(A)$ for every integrally closed $\mathfrak{m}$-primary ideal I in A.

Proof. Let $I$ be an integrally closed $\mathfrak{m}$-primary ideal in $A$, and choose a desingularization $f: X \rightarrow \operatorname{Spec} A$ so that the ideal $I$ is represented by an anti-nef divisor $Z$ on $X$. Then it is known (and easy to show, applying the formula (7.2) (1) to $D=-n Z$, since $\left.\overline{I^{n}}=\mathrm{H}^{0}\left(X, \mathcal{O}_{X}(D)\right)\right)$ that $\mathrm{e}_{I}^{0}(A)=-Z^{2}>0$. By (7.2) (2) we have $2 \ell_{A}(A / I)-\mathrm{e}_{I}^{0}(A)=-K_{X} Z$ if $A$ is a rational local ring, while by (7.4) (1) (resp. (2)) we have $-K_{X} Z>0$ (resp. $-K_{X} Z \geq 0$ ) if $A$ is a regular local ring (resp. a Gorenstein rational local ring). Hence the only if parts of assertions (1) and (2) follow. Conversely, assume that $A$ is not a Gorenstein rational local ring, and take a divisor $Z$ on $X$ so that $-Z$ is ample. Then we get the reverse inequality $\ell_{A}(A / I)<\frac{1}{2} \mathrm{e}_{I}^{0}(A)$ for the ideal $I=\mathrm{H}^{0}\left(X, \mathcal{O}_{X}(-n Z)\right)$ in $A$ with a sufficiently large integer $n$, which proves the if part of assertion (2). 
We need the following.

Fact (7.6). Let $E$ be an exceptional curve on $X$. Then we say that $E$ is a $(-1)$ curve, if $E \cong \mathbb{P}_{k}^{1}$ and $E^{2}=-1$. It is known that for each $(-1)$ curve $E$ on $X$ the morphism $f: X \rightarrow \operatorname{Spec} A$ is decomposed as $f=\pi \circ g$ so that (1) $\pi: X \rightarrow X^{\prime}$ is the contraction of $E$, that is, $\pi(E)$ is a point and $\pi$ is an isomorphism on $X-E$, and (2) $g: X^{\prime} \rightarrow \operatorname{Spec} A$ is a morphism of schemes with $X^{\prime}$ regular. We say that $X$ is a minimal resolution of $\operatorname{Spec} A$ if $X$ contains no $(-1)$ curves.

Fact (7.7). Let $\pi: X \rightarrow X^{\prime}$ be the contraction of a $(-1)$ curve $E$ on $X$. Let $Z=\sum_{1=1}^{r} n_{i} E_{i}^{\prime}$ be an exceptional anti-nef divisor on $X^{\prime}$ and let $E_{i}$ denote, for each $1 \leq i \leq r$, a proper transform of $E_{i}^{\prime}$ on $X$, that is, the unique irreducible curve with $\pi\left(E_{i}\right)=E_{i}^{\prime}$. Then the total transform of $Z$ is the divisor $\pi^{*}(Z)=$ $\sum_{1=1}^{r} n_{i} E_{i}+m E$ on $X$, where $m$ denotes the integer determined by the condition $\pi^{*}(Z) E=0$. It is easy to see that $\pi^{*}(Z)$ is also anti-nef and $\mathrm{H}^{0}\left(X^{\prime}, O_{X^{\prime}}(-Z)\right)=$ $\mathrm{H}^{0}\left(X, O_{X}\left(-\pi^{*}(Z)\right)\right)$. Conversely, let $\bar{Z}=\sum_{1=1}^{r} n_{i} E_{i}+m E$ be an exceptional divisor on $X$. Then $\bar{Z} E=0$ if and only if $\bar{Z}=\pi^{*}(Z)$, where $Z=\sum_{1=1}^{r} n_{i} E_{i}^{\prime}$. Similarly, let $p=\pi_{r} \circ \pi_{r-1} \circ \ldots \circ \pi_{1}: X \rightarrow X_{1} \rightarrow \ldots \rightarrow X_{r}$ be a composition of contractions of $(-1)$ curves and let $E_{i}$ denote the proper transform of the $(-1)$ curve contracted by $\pi_{i}$. Let $\bar{Z}=\sum_{1=1}^{r} n_{i} E_{i}+m E$ be an exceptional divisor on $X$. Then $\bar{Z}=p^{*}(Z)$ if and only if $\bar{Z} E_{i}=0$ for all $1 \leq i \leq r$.

We now come to the main result of this section.

Theorem (7.8). Let $A$ be a two-dimensional Gorenstein excellent rational local ring. Let $I$ be an $\mathfrak{m}$-primary ideal in $A$, and assume that $I$ contains a parameter ideal $Q$ in $A$ as a reduction. Then $I$ is a good ideal in $A$ if and only if $I$ is an integrally closed ideal represented on the minimal resolution of $\operatorname{Spec} A$.

Proof. By (2.5) we may assume $I$ is integrally closed. Hence $I=\mathrm{H}^{0}\left(X, O_{X}(-Z)\right)$ for some desingularization $X$ of Spec $A$ and some anti-nef divisor $Z$ on $X$. By (2.2) the ideal $I$ is good if and only if $\ell_{A}(A / I)=\frac{1}{2} \mathrm{e}_{I}^{0}(A)$. By the proof of (7.5) the latter condition is equivalent to saying that $K_{X} Z=0$. Let $g: X \rightarrow \bar{X}$ be the unique morphism to the minimal resolution $\bar{X}$ of $\operatorname{Spec} A$. Then $K_{\bar{X}}=0$, which follows from the fact that every $E_{i}$ on the minimal resolution $X$ satisfies the conditions $p_{a}\left(E_{i}\right)=0$ and $E_{i}^{2}=-2$. Let $K_{X}=\sum_{i=1}^{r} a_{i} E_{i} \geq 0$, and notice that $a_{i}>0$ if and only if $E_{i}$ is contracted by $g$. Then, because $Z$ is anti-nef and $K_{X} \geq 0, K_{X} Z=0$ if and only if $Z E_{i}=0$ for every $E_{i}$ with $a_{i}>0$. Therefore by (7.7) $I$ is a good ideal in $A$ if and only if $Z$ is a total transform of some divisor $Z^{\prime}$ on $\bar{X}$, that is, by definition, $I$ is represented on $\bar{X}$.

As a consequence of Theorem (7.8) we get the following, which shows that the good ideals in two-dimensional Gorenstein rational local rings are preserved by taking products.

Corollary (7.9). Let $A$ be a two-dimensional Gorenstein excellent rational local ring, and assume the field $A / \mathfrak{m}$ is infinite. Let $I$ and $J$ be $\mathfrak{m}$-primary integrally closed ideals in $A$. Then $I J$ is a good ideal in $A$ if and only if both $I$ and $J$ are good ideals in $A$.

Proof. Since $A / \mathfrak{m}$ is infinite, every $\mathfrak{m}$-primary ideal contains a parameter ideal in $A$ as a reduction. The product $I J$ is an integrally closed ideal in $A((7.3)(1))$. Hence 
by (7.8) we may assume that

$$
I=\mathrm{H}^{0}\left(X, \mathcal{O}_{X}(-Z)\right), \quad J=\mathrm{H}^{0}\left(X, \mathcal{O}_{X}\left(-Z^{\prime}\right)\right), \quad I J=\mathrm{H}^{0}\left(X, \mathcal{O}_{X}\left(-\left(Z+Z^{\prime}\right)\right)\right),
$$

where $Z$ and $Z^{\prime}$ are anti-nef divisors on some resolution $X$ of $\operatorname{Spec} A$. Then $K_{X}\left(Z+Z^{\prime}\right)=0$ if and only if $K_{X} Z=K_{X} Z^{\prime}=0$. Thus from (7.8) the equivalence readily follows.

To close this section let us explore the examples stated at the beginning. Here we deal with the two simplest examples. However, it is possible by the same method to explicitly describe the good ideals for any two-dimensional Gorenstein rational excellent local rings.

Example (7.10). Let $k[[X, Y, Z]]$ be the formal power series ring over a field $k$.

(1) Let $A=k[[X, Y, Z]] /\left(Z^{2}-X Y\right)$. Then the exceptional set of the minimal resolution $X$ of $\operatorname{Spec} A$ is a single irreducible curve $E$. Hence the good ideals in $A$ are just $\mathrm{H}^{0}\left(X, \mathcal{O}_{X}(-n E)\right)=\mathfrak{m}^{n}$ with $n \geq 1$.

(2) Let $A=k[[X, Y, Z]] /\left(Z^{3}-X Y\right)$. Let $x, y$, and $z$ denote respectively the reduction of $X, Y$, and $Z \bmod$ the ideal $\left(Z^{3}-X Y\right)$. Then the exceptional set of the minimal resolution $X$ of $\operatorname{Spec} A$ consists of two irreducible curves $E_{1}, E_{2}$ such that $E_{1}^{2}=E_{2}^{2}=-2$ and $E_{1} E_{2}=1$. Hence $Z=a E_{1}+b E_{2}(a, b>0)$ is anti-nef if and only if $2 a \geq b$ and $2 b \geq a$. It is routine to check that such pairs $(a, b)$ are the sum of copies of $(1,1),(2,1)$, and $(1,2)$. Also, letting $\operatorname{div}_{X}(x)=2 E_{1}+E_{2}, \operatorname{div}_{X}(y)=E_{1}+2 E_{2}$, and $\operatorname{div}_{X}(z)=E_{1}+E_{2}$, we have

$$
\begin{gathered}
\mathrm{H}^{0}\left(X, \mathcal{O}_{X}\left(-\left(E_{1}+E_{2}\right)\right)=\mathfrak{m},\right. \\
\mathrm{H}^{0}\left(X, \mathcal{O}_{X}\left(-\left(2 E_{1}+E_{2}\right)\right)=\left(x, y^{2}, y z, z^{2}\right),\right. \\
\mathrm{H}^{0}\left(X, \mathcal{O}_{X}\left(-\left(E_{1}+2 E_{2}\right)\right)=\left(x^{2}, x z, z^{2}, y\right) .\right.
\end{gathered}
$$

These three ideals are good in $A$, and every good ideal in $A$ is the product of copies of these ideals.

\section{Monomial good ideals in the polynomial Ring $k\left[X_{1}, X_{2}, X_{3}\right]$ OVER A FIELD $k$ WHEN $\operatorname{dim} A \geq 3$}

In this section we shall prove the following.

Proposition (8.1). Suppose that $A$ contains a field. Then $\mathcal{X}_{A}$ is infinite if $\operatorname{dim} A$ $\geq 3$.

The assertion is reduced to the case where $A=k\left[\left[X_{1}, X_{2}, X_{3}\right]\right]$ is the formal power series ring in three variables over a field $k$. And in this section we focus our attention on the analysis of good ideals generated by monomials in $X_{1}, X_{2}, X_{3}$ in the polynomial ring $R=k\left[X_{1}, X_{2}, X_{3}\right]$. The purpose is to show there exist plenty of monomial good ideals contained in $R$.

Let $k$ be a field and let $R=k\left[X_{1}, X_{2}, X_{3}\right]$ be the polynomial ring. Let $H=\mathbb{Z}^{3}$ with $\left\{e_{1}, e_{2}, e_{3}\right\}$ the standard basis. Let $L=\left\{\left(\alpha_{1}, \alpha_{2}, \alpha_{3}\right) \in H \mid \alpha_{i} \geq 0\right\}$. We regard $R$ as an $H$-graded ring whose grading is given by $R_{0}=k, R_{e_{i}} \ni X_{i}$ for $i=1,2,3$. Therefore, graded ideals of $R$ are just ideals generated by monomials in $X_{1}, X_{2}, X_{3}$. Let $\mathfrak{M}=\left(X_{1}, X_{2}, X_{3}\right)$ and $A=R_{\mathfrak{M}}$. For each $\alpha \in L$ let $\alpha_{i}$ denote the $i \frac{\text { th }}{}$ entry of $\alpha$, whence $\alpha=\left(\alpha_{1}, \alpha_{2}, \alpha_{3}\right)$. We denote the monomial $X_{1}^{\alpha_{1}} X_{2}^{\alpha_{2}} X_{3}^{\alpha_{3}}$ simply by $X^{\alpha}$. Let $a_{1}, a_{2}, a_{3} \geq 1$ be fixed integers and put $Q=\left(X_{1}^{a_{1}}, X_{2}^{a_{2}}, X_{3}^{a_{3}}\right)$. 
Let $J$ denote a graded ideal in $R$ with $N=\mu_{R}(J)$. Then there exists a unique subset $\Lambda \subseteq L$ such that $\sharp \Lambda=N$ and $J=\left(X^{\lambda} \mid \lambda \in \Lambda\right)$. Let us refer to this set $\Lambda=\Lambda_{J}$ as the $m$-basis of $J$.

The next three results are well-known.

Lemma (8.2). Let $\alpha \in L$. Then $X^{\alpha} \in \bar{J}$ if and only if $\left(X^{\alpha}\right)^{p} \in J^{p}$ for some $p \geq 1$.

Corollary (8.3). Let $\alpha \in L$. Then $X^{\alpha} \in \bar{Q}$ if and only if $\frac{\alpha_{1}}{a_{1}}+\frac{\alpha_{2}}{a_{2}}+\frac{\alpha_{3}}{a_{3}} \geq 1$. Hence

$$
\bar{Q}=\left(X^{\alpha} \mid \alpha \in L, \frac{\alpha_{1}}{a_{1}}+\frac{\alpha_{2}}{a_{2}}+\frac{\alpha_{3}}{a_{3}} \geq 1\right)
$$

Lemma (8.4). Let $\alpha \in L$. Then

$$
Q: X^{\alpha}= \begin{cases}\left(X_{1}^{a_{1}-\alpha_{1}}, X_{2}^{a_{2}-\alpha_{2}}, X_{3}^{a_{3}-\alpha_{3}}\right) & \text { if } a_{i}>\alpha_{i} \text { for all } i \\ R & \text { otherwise }\end{cases}
$$

Definition (8.5). Our graded ideal $J$ is said to be $Q$-good if $J \supseteq Q, J^{2}=Q J$, and $J=Q: J$.

Let $\mathcal{X}_{Q}$ be the set of $Q$-good ideals in $R$. In this section we are interested in the structure of the set $\mathcal{X}_{Q}$, and especially in the question when $\mathcal{X}_{Q} \neq \varnothing$. Notice that $\mathcal{X}_{Q}$ is necessarily finite. We shall often denote $\mathcal{X}_{Q}$ simply by $\mathcal{X}_{\left(a_{1}, a_{2}, a_{3}\right)}$. Our destination is Theorem (8.25) below. We begin with the following.

Lemma (8.6). (1) Let $J \in \mathcal{X}_{Q}$. Then $Q \subsetneq J \subseteq \mathfrak{M}$ and $J A \in \mathcal{X}_{A}$.

(2) Let $J \in \mathcal{X}_{Q}$. Then $J \subseteq \bar{Q}$.

(3) Let $J, K \in \mathcal{X}_{Q}$. Then $J=K$ if $J \subseteq K$.

(4) $2 \mid a_{1} a_{2} a_{3}$ if $\mathcal{X}_{Q} \neq \varnothing$.

Proof. Assertions (1) and (2) are clear and well-known. See (2.4) for assertion (3). Assertion (4) follows from $(2.2)$, since $\ell_{R}(R / Q)=a_{1} a_{2} a_{3}$.

We note here the following.

Example (8.7). $\mathcal{X}_{(2,2,2)}=\left\{\mathfrak{M}^{2}\right\}$.

Proof. Let $Q=\left(X_{1}^{2}, X_{2}^{2}, X_{3}^{2}\right)$. Then $\mathfrak{M}^{2} \subseteq \bar{Q}$ since $\mathfrak{M}^{4}=Q \mathfrak{M}^{2}$. Therefore $\bar{Q}=$ $\mathfrak{M}^{2}$, as $\bar{Q} \subseteq \mathfrak{M}^{2}$ by $(8.3)$. Because $\ell_{R}\left(R / \mathfrak{M}^{2}\right)=4$ and $\ell_{R}(R / Q)=8$, we get $\mathfrak{M}^{2} \in \mathcal{X}_{Q}$, so that $\mathcal{X}_{Q}=\left\{\mathfrak{M}^{2}\right\}$ by $(8.6)(2),(3)$.

Proposition (8.8). Let $J \in \mathcal{X}_{Q}$ with $\Lambda=\Lambda_{J}$. Then the following assertions hold true.

(1) $\Lambda \supsetneq\left\{a_{1} e_{1}, a_{2} e_{2}, a_{3} e_{3}\right\}$.

(2) Let $\lambda \in \Lambda$ and assume that $\lambda=c_{i}$ for some $1 \leq i \leq 3$ and $0 \leq c \in \mathbb{Z}$. Then $c=a_{i}$ and $\lambda=a_{i} e_{i}$.

(3) Let $\alpha \in L$. Then $X^{\alpha} \in J^{2}$ if and only if $\alpha=\beta+\lambda+a_{i} e_{i}$ for some $\beta \in L$, $\lambda \in \Lambda$, and $1 \leq i \leq 3$.

(4) $a_{i}=\min \left\{0<\ell \in \mathbb{Z} \mid X_{i}^{\ell} \in J\right\}$ for all $1 \leq i \leq 3$.

Proof. (1) Since $X_{i}^{a_{i}} \in Q \subseteq J=\left(X^{\lambda} \mid \lambda \in \Lambda\right)$, we have $a_{i} e_{i}=\alpha+\lambda$ for some $\alpha \in L$ and $\lambda \in \Lambda$. Then $a_{i} \geq \lambda_{i}$, since $a_{i}=\alpha_{i}+\lambda_{i}$. On the other hand, as $X^{\lambda}=X_{i}^{\lambda_{i}} \in \bar{Q}$ 
by (8.6)(2) (note that $\lambda=\lambda_{i} e_{i}$ ), we have $\lambda_{i} / a_{i} \geq 1$ by (8.3). Hence $\lambda_{i}=a_{i}$ so that $\lambda=a_{i} e_{i}$. Thus $\Lambda \supseteq\left\{a_{1} e_{1}, a_{2} e_{2}, a_{3} e_{3}\right\}$. The inclusion is strict since $J \supsetneq Q$.

(2) Since $X^{\lambda}=X_{i}^{c} \in J$, we have $c \geq a_{i}$ by (8.3) and (8.6)(2). If $c>a_{i}$, then $\lambda \neq a_{i} e_{i}$ but $X^{\lambda} \in Q$, which violates by (1) the fact that $\left\{X^{\lambda}\right\}_{\lambda \in \Lambda}$ forms a minimal system of generators for the ideal $J$. Hence $c=a_{i}$ and $\lambda=a_{i} e_{i}$.

(3) Notice that $J^{2}=Q J$.

(4) Let $\ell>0$ be an integer with $X_{i}^{\ell} \in J$. Then $\ell e_{i}=\alpha+\lambda$ for some $\alpha \in L$ and $\lambda \in \Lambda$. Hence $\lambda=\lambda_{i} e_{i}$. Therefore by (2) $\lambda_{i}=a_{i}$ whence $\ell=\alpha_{i}+\lambda_{i} \geq a_{i}$.

Corollary (8.9). Let $b_{1}, b_{2}, b_{3} \geq 1$ be integers. Then $a_{i}=b_{i}$ for all $1 \leq i \leq 3$ if $\mathcal{X}_{\left(a_{1}, a_{2}, a_{3}\right)} \cap \mathcal{X}_{\left(b_{1}, b_{2}, b_{3}\right)} \neq \varnothing$.

Proof. See (8.8)(4).

For a while (until Corollary (8.14), below) let $J \in \mathcal{X}_{Q}$ and $\Lambda=\Lambda_{J}$. We put $\Gamma=\Lambda \backslash\left\{a_{1} e_{1}, a_{2} e_{2}, a_{3} e_{3}\right\}$. Then $\Lambda \supsetneq \Gamma \neq \varnothing$ and $J=Q+\left(X^{\gamma} \mid \gamma \in \Gamma\right)$. Notice that $X^{\gamma} \notin Q$ for any $\gamma \in \Gamma$, because $\left\{X^{\lambda}\right\}_{\lambda \in \Lambda}$ is a minimal system of generators for the ideal $J$. For each $1 \leq i \leq 3$ we put $\Gamma_{i}=\left\{\gamma \in \Gamma \mid \gamma_{i}=0\right\}$ and $\Gamma^{\prime}=\Gamma \backslash \bigcup_{i=1}^{3} \Gamma_{i}$. Then we have the following.

Lemma (8.10). (1) $\Gamma_{i} \neq \varnothing$ for any $1 \leq i \leq 3$.

(2) $\Gamma_{i} \cap \Gamma_{j}=\varnothing$ if $i \neq j$.

(3) Let $\alpha=\left(0, \alpha_{2}, \alpha_{3}\right) \in \Gamma_{1}$. Then $0<\alpha_{2}<a_{2}$ and $0<\alpha_{3}<a_{3}$.

(4) Let $\alpha=\left(0, \alpha_{2}, \alpha_{3}\right), \beta=\left(0, \beta_{2}, \beta_{3}\right) \in \Gamma_{1}$. Then $\alpha=\beta$ if $\alpha_{2} \leq \beta_{2}$ and $\alpha_{3} \leq \beta_{3}$. Hence $\alpha_{2}>\beta_{2}$ if and only if $\alpha_{3}<\beta_{3}$.

Proof. (1) Assume that $\Gamma_{i}=\varnothing$ for some $1 \leq i \leq 3$. Then, since $\gamma_{i}>0$ for all $\gamma \in \Gamma$, we have $X_{i}^{a_{i}-1} X^{\gamma} \in Q$ for all $\gamma \in \Gamma$, so that $X_{i}^{a_{i}-1} \in Q: J=J$, which is impossible (cf. (8.8)(4)).

(2) Assume that $\Gamma_{1} \cap \Gamma_{2} \neq \varnothing$ and choose $\gamma=\left(0,0, \gamma_{3}\right) \in \Gamma_{1} \cap \Gamma_{2}$. Then by (8.8)(2) we get $\gamma=a_{3} e_{3}$, which is absurd since $\gamma \in \Gamma=\Lambda \backslash\left\{a_{1} e_{1}, a_{2} e_{2}, a_{3} e_{3}\right\}$. Thus $\Gamma_{1} \cap \Gamma_{2}=\varnothing$. Similarly we have $\Gamma_{i} \cap \Gamma_{j}=\varnothing$ if $i \neq j$.

(3) and (4) By (2) we have $0<\alpha_{2}$ and $0<\alpha_{3}$. If $\alpha_{2} \geq a_{2}$, then $\alpha \neq a_{2} e_{2}$ but $X_{2}^{a_{2}} \mid X^{\alpha}$ in $R$. Therefore the set $\left\{X^{\lambda}\right\}_{\lambda \in \Lambda \backslash\{\alpha\}}$ is still a system of generators for $J$, which violates the minimality of $\Lambda$. Thus $\alpha_{2}<a_{2}$. Similarly we have $\alpha_{3}<a_{3}$. If $\alpha \neq \beta, \alpha_{2} \leq \beta_{2}$, and $\alpha_{3} \leq \beta_{3}$, then the set $\left\{X^{\lambda}\right\}_{\lambda \in \Lambda \backslash\{\beta\}}$ is still a system of generators for $J$. Thus $\alpha=\beta$ if $\alpha_{2} \leq \beta_{2}$ and $\alpha_{3} \leq \beta_{3}$.

Corollary (8.11). $\mu_{R}(J) \geq \sum_{i=1}^{3} \sharp \Gamma_{i}+3 \geq 6$.

Proof. Since $\sum_{i=1}^{3} \sharp \Gamma_{i} \geq 3$ by $(8.10)(1)$, we get $\mu_{R}(J)=\sharp \Lambda \geq \sum_{i=1}^{3} \sharp \Gamma_{i}+3 \geq 6$ by $(8.10)(2)$.

We put

$$
\begin{array}{ll}
a=\min \left\{\gamma_{1} \mid \gamma \in \Gamma_{3}\right\}, \quad b=\min \left\{\gamma_{2} \mid \gamma \in \Gamma_{1}\right\}, & c=\min \left\{\gamma_{3} \mid \gamma \in \Gamma_{2}\right\}, \\
a^{\prime}=\min \left\{\gamma_{1} \mid \gamma \in \Gamma_{2}\right\}, \quad b^{\prime}=\min \left\{\gamma_{2} \mid \gamma \in \Gamma_{3}\right\}, & c^{\prime}=\min \left\{\gamma_{3} \mid \gamma \in \Gamma_{1}\right\} .
\end{array}
$$

Then $0<a, a^{\prime}<a_{1}, 0<b, b^{\prime}<a_{2}$, and $0<c, c^{\prime}<a_{3}$. We furthermore have the following.

Lemma (8.12). $a+a^{\prime}=a_{1}, b+b^{\prime}=a_{2}$, and $c+c^{\prime}=a_{3}$. 
Proof. Choose $(a, k, 0) \in \Gamma_{3}$ and $\left(a^{\prime}, 0, \ell\right) \in \Gamma_{2}$. Then we have $X_{1}^{a+a^{\prime}} X_{2}^{k} X_{3}^{\ell} \in J^{2}$. Therefore by (8.8)(3) $\left(a+a^{\prime}, k, \ell\right)=\beta+\lambda+a_{i} e_{i}$ for some $\beta \in L, \lambda \in \Lambda$, and $1 \leq i \leq 3$. We have $i=1$, because $k<a_{2}$ and $\ell<a_{3}$ by (8.10)(3). Hence $a+a^{\prime} \geq a_{1}$. Assume that $a+a^{\prime}>a_{1}$ and let $f=X_{1}^{a-1} X_{2}^{a_{2}-1}$. Then $f X^{\gamma} \in Q$ for all $\gamma \in \Gamma$. In fact, we may assume $\gamma \in \Gamma_{2}$. Then, because $\gamma_{1} \geq a^{\prime}$ by the definition of $a^{\prime}$, we have $\gamma_{1}+a-1 \geq a^{\prime}+a-1 \geq a_{1}$, whence $f X^{\gamma} \in\left(X_{1}^{a_{1}}\right) \subseteq Q$. Consequently $f \in Q: J=J$, and so $\left(a-1, a_{2}-1,0\right)=\beta+\lambda$ for some $\beta \in L$ and $\lambda \in \Lambda$. Then $\lambda_{1}<a<a_{1}, \lambda_{2}<a_{2}$, and $\lambda_{3}=0$. Hence $\lambda \in \Gamma_{3}$, so $\lambda_{1} \geq a$ by the definition of $a$, which is impossible. Thus $a+a^{\prime}=a_{1}$. We have $b+b^{\prime}=a_{2}$ and $c+c^{\prime}=a_{3}$ by the symmetry.

Corollary (8.13). $\min \left\{a_{1}, a_{2}, a_{3}\right\} \geq 2$.

Let $a=\left(a_{1}, a_{2}, a_{3}\right)$ and $e=(1,1,1)$. Then we have the following.

Proposition (8.14). $J: \mathfrak{M}=J+\left(X^{a-e-\gamma} \mid \gamma \in \Gamma\right)$.

Proof. Since $J=Q+\left(X^{\gamma} \mid \gamma \in \Gamma\right)$ and $J=Q: J$, we have $J=\bigcap_{\gamma \in \Gamma}\left(Q: X^{\gamma}\right)$. Because $X^{\gamma} \notin Q$ for any $\gamma \in \Gamma$, by (8.4) we get $Q: X^{\gamma}=\left(X_{1}^{a_{1}-\gamma_{1}}, X_{2}^{a_{2}-\gamma_{2}}, X_{3}^{a_{3}-\gamma_{3}}\right)$. Hence the socle of $R /\left[Q: X^{\gamma}\right]$ is generated by the element $X^{a-e-\gamma} \bmod Q: X^{\gamma}$. On the other hand we have $\mathrm{t}(R / J)=\sharp \Gamma$, since $r(R / J)=\mu_{R}(J / Q)$ (cf. (2.2)). Therefore, applying the functor $\operatorname{Hom}_{R}(R / \mathfrak{M}, *)$ to the embedding $0 \rightarrow R / J \rightarrow$ $\bigoplus_{\gamma \in \Gamma} R /\left[Q: X^{\gamma}\right]$, we have the isomorphisms

$$
\begin{aligned}
\operatorname{Hom}_{R}(R / \mathfrak{M}, R / J) & \cong \bigoplus_{\gamma \in \Gamma} \operatorname{Hom}_{R}\left(R / \mathfrak{M}, R /\left[Q: X^{\gamma}\right]\right) \\
& \cong \bigoplus_{\gamma \in \Gamma} \frac{k X^{a-e-\gamma}+\left[Q: X^{\gamma}\right]}{Q: X^{\gamma}}
\end{aligned}
$$

of graded $R$-modules. It follows that $J: \mathfrak{M}$ is generated by the elements of degree $\{a-e-\gamma\}_{\gamma \in \Gamma}$, so that we have $J: \mathfrak{M}=J+\left(X^{a-e-\gamma} \mid \gamma \in \Gamma\right)$.

Lemma (8.15). Suppose that $\mathcal{X}_{\left(a_{1}, a_{2}, a_{3}\right)} \neq \varnothing$. Then $\mathcal{X}_{\left(a_{1} k, a_{2} \ell, a_{3} m\right)} \neq \varnothing$ for all integers $k, \ell, m \geq 1$.

Proof. Let $\varphi: R \rightarrow S:=R$ be the $k$-algebra map defined by $\varphi\left(X_{1}\right)=X_{1}^{k}, \varphi\left(X_{2}\right)=$ $X_{2}^{\ell}$, and $\varphi\left(X_{3}\right)=X_{3}^{m}$. We choose $J \in \mathcal{X}_{Q}$. Then $(J S)^{2}=Q S \cdot J S$ and $J S=$ $Q S: J S$, since $\varphi$ is flat. Hence $\mathcal{X}_{\left(a_{1} k, a_{2} \ell, a_{3} m\right)} \neq \varnothing$, as $Q S=\left(X_{1}^{a_{1} k}, X_{2}^{a_{2} \ell}, X_{3}^{a_{3} m}\right)$ in $S$.

We are now ready to study the structure of the sets $\mathcal{X}_{Q}$ for the given ideals $Q=\left(X_{1}^{a_{1}}, X_{2}^{a_{2}}, X_{3}^{a_{3}}\right)$ in $R$. First let us note the following, in which we maintain the same notation as in Lemma (8.12).

Proposition (8.16). Let $J \in \mathcal{X}_{Q}$. Then the following conditions are equivalent.

(1) $\mu_{R}(J)=6$.

(2) $\sharp \Gamma_{i}=1$ for all $1 \leq i \leq 3$.

(3) $2 \mid a_{i}$ for all $1 \leq i \leq 3$, and $J=\left(X_{1}^{a_{1} / 2}, X_{2}^{a_{2} / 2}, X_{3}^{a_{3} / 2}\right)^{2}$.

Proof. (3) $\Rightarrow(1)$ and (1) $\Rightarrow(2)$ are clear (cf. (8.10) and (8.11)).

$(2) \Rightarrow(3)$ Since each $\Gamma_{i}$ is a singleton, we have $\Gamma_{1}=\left\{\left(0, b, c^{\prime}\right)\right\}, \Gamma_{2}=\left\{\left(a^{\prime}, 0, c\right)\right\}$, and $\Gamma_{3}=\left\{\left(a, b^{\prime}, 0\right)\right\}$. Hence $X_{1}^{a} X_{2}^{b+b^{\prime}} X_{3}^{c^{\prime}} \in J^{2}$. By (8.8)(3) we may write

$$
\left(a, b+b^{\prime}, c^{\prime}\right)=\beta+\lambda+a_{i} e_{i}
$$


with $\beta \in L, \lambda \in \Lambda$, and $1 \leq i \leq 3$. Then, since $a<a_{1}$ and $c^{\prime}<a_{3}$, we have $i=2$. Because $b+b^{\prime}=a_{2}$ by (8.12), we get $\left(a, 0, c^{\prime}\right)=\beta+\lambda$. Hence $\lambda \notin\left\{a_{1} e_{1}, a_{2} e_{2}, a_{3} e_{3}\right\}$, as $\lambda_{1} \leq a<a_{1}, \lambda_{2}=0$, and $\lambda_{3} \leq c^{\prime}<a_{3}$. Therefore $\lambda \in \Gamma_{2}$, and so $\lambda=\left(a^{\prime}, 0, c\right)$, whence $\left(a, 0, c^{\prime}\right)=\beta+\left(a^{\prime}, 0, c\right)$. Thus $a \geq a^{\prime}$ and $c^{\prime} \geq c$. Similarly, because $X_{1}^{a^{\prime}} X_{2}^{b} X_{3}^{c+c^{\prime}} \in J^{2}$ and $c+c^{\prime}=a_{3}$, we get $\left(a^{\prime}, b, 0\right)=\beta+\left(a, b^{\prime}, 0\right)$ for some $\beta \in L$. Hence $a^{\prime} \geq a$ and $b \geq b^{\prime}$. As $X_{1}^{a+a^{\prime}} X_{2}^{b^{\prime}} X_{3}^{c} \in J^{2}$, we also have $b^{\prime} \geq b$ and $c \geq c^{\prime}$. Therefore $a=a^{\prime}, b=b^{\prime}$, and $c=c^{\prime}$, whence $a_{1}=2 a, a_{2}=2 b$, and $a_{3}=2 c$. We get $\left(X_{1}^{a}, X_{2}^{b}, X_{3}^{c}\right)^{2} \in \mathcal{X}_{Q}$ by (8.7) and the proof of (8.15). Thus by (8.6)(3) $J=\left(X_{1}^{a}, X_{2}^{b}, X_{3}^{c}\right)^{2}$, since $J \supseteq\left(X_{1}^{a}, X_{2}^{b}, X_{3}^{c}\right)^{2}$.

Corollary (8.17). The following conditions are equivalent.

(1) $2 \mid a_{i}$ for all $1 \leq i \leq 3$.

(2) $\mu_{R}(J)=6$ for some $J \in \mathcal{X}_{Q}$.

Proof. The implication $(1) \Rightarrow(2)$ follows from the fact that $\left(X_{1}^{\frac{a_{1}}{2}}, X_{2}^{\frac{a_{2}}{2}}, X_{3}^{\frac{a_{3}}{2}}\right)^{2} \in$ $\mathcal{X}_{Q}$ (cf. (8.7) and the proof of (8.15)).

Corollary (8.18). Let $n \geq 1$ be an integer. Then $\mathcal{X}_{(2,2, n)} \neq \varnothing$ if and only if $2 \mid n$. When this is the case, $\mathcal{X}_{(2,2, n)}=\left\{\left(X_{1}, X_{2}, X_{3}^{\frac{n}{2}}\right)^{2}\right\}$.

Proof. Let $\alpha=\left(0, \alpha_{2}, \alpha_{3}\right), \beta=\left(0, \beta_{2}, \beta_{3}\right) \in \Gamma_{1}$. Then $\alpha_{2}=\beta_{2}=1$ by $(8.10)(3)$ as $a_{2}=2$, whence $\alpha=\beta$ by $(8.10)(4)$, so that $\sharp \Gamma_{1}=1$. Similarly we have $\sharp \Gamma_{2}=$ $\sharp \Gamma_{3}=1$. Thus by $(8.16)(2)$ we get $2 \mid n$ and $J=\left(X_{1}, X_{2}, X_{3}^{\frac{n}{2}}\right)^{2}$. See (8.17) for the if part.

Here let us note the following proof of Proposition (8.1).

Proof of Proposition (8.1). As $\sharp \mathcal{X}_{A}=\sharp \mathcal{X}_{\widehat{A}}$ by (2.1), we may assume that $\widehat{A}=A$. Let $k$ denote a coefficient field of $A$. Then $A$ contains the formal power series ring $P=k\left[\left[X_{1}, X_{2}, \ldots, X_{d}\right]\right](d=\operatorname{dim} A)$, so that $A$ is a finitely generated free $P$-module. Since $I A \in \mathcal{X}_{A}$ and $I=I A \cap P$ for all $I \in \mathcal{X}_{P}$, it suffices to show that $\mathcal{X}_{P}$ is infinite. Let $S=k\left[\left[X_{1}, X_{2}, X_{3}\right]\right]$. Then for all $I \in \mathcal{X}_{S}$ we naturally have $I P+\left(X_{4}, \ldots, X_{d}\right) P \in \mathcal{X}_{P}$. Hence $\mathcal{X}_{A}$ is infinite, because $\mathcal{X}_{S}$ is infinite by (2.1) and (8.18).

Let us continue the analysis of the set $\mathcal{X}_{Q}$.

Proposition (8.19). $\mu_{R}(J) \neq 7$ for any $J \in \mathcal{X}_{Q}$.

Proof. Let $J \in \mathcal{X}_{Q}$ and assume that $\mu_{R}(J)=7$. Then by (8.16) $\sharp \Gamma_{i} \geq 2$ for some $i$. On the other hand, $\sharp \Gamma=4$ and $\Gamma_{j} \neq \varnothing$ for all $1 \leq j \leq 3$ by (8.10) and (8.11). Hence we may assume $\sharp \Gamma_{1}=2$ and $\sharp \Gamma_{2}=\sharp \Gamma_{3}=1$. Let $\Gamma_{1}=\left\{(0, b, k),\left(0, \ell, c^{\prime}\right)\right\}$, $\Gamma_{2}=\left\{\left(a^{\prime}, 0, c\right)\right\}$, and $\Gamma_{3}=\left\{\left(a, b^{\prime}, 0\right)\right\}$. Here $a, b, c, a^{\prime}, b^{\prime}$, and $c^{\prime}$ denote the integers used in Lemma (8.12). Hence $1 \leq b<\ell<a_{2}$ and $1 \leq c^{\prime}<k<a_{3}$ by (8.10)(3), (4). We divide the proof into six steps.

Claim 1. $a^{\prime} \geq \frac{a_{1}}{2} \geq a$.

Proof of Claim 1. Since $\left(X_{1}^{a^{\prime}} X_{3}^{c}\right)^{2}=X_{1}^{2 a^{\prime}} X_{2}^{2 c} \in J^{2}$, by (8.8)(3) $\left(2 a^{\prime}, 0,2 c\right)=\beta+$ $\lambda+a_{i} e_{i}$ for some $\beta \in L, \lambda \in \Lambda$, and $1 \leq i \leq 3$. Hence, if $2 a^{\prime}<a_{1}$, we have $i=3$ and $\left(2 a^{\prime}, 0,2 c-a_{3}\right)=\beta+\lambda$. Because $2 c-a_{3}<a_{3}$ (recall that $c<a_{3}$ ) and $2 a^{\prime}<a_{1}$, we have $\lambda_{1}<a_{1}, \lambda_{2}=0$, and $\lambda_{3}<a_{3}$. Hence $\lambda \in \Gamma_{2}$, so that 
$\left(2 a^{\prime}, 0,2 c-a_{3}\right)=\beta+\left(a^{\prime}, 0, c\right)$. Hence $2 c-a_{3}=\beta_{3}+c \geq c$, so that $c \geq a_{3}$, which is absurd. Therefore $a^{\prime} \geq \frac{a_{1}}{2}$ whence $\frac{a_{1}}{2} \geq a$, as $a+a^{\prime}=a_{1}$ by (8.12).

Claim 2. $b^{\prime} \geq \frac{a_{2}}{2} \geq b$.

Proof of Claim 2. This is proved similarly as Claim 1. Use the fact that $\left(X_{1}^{a} X_{2}^{b^{\prime}}\right)^{2}$ $=X_{1}^{2 a} X_{2}^{2 b^{\prime}} \in J^{2}$.

Claim 3. $a^{\prime}=a=\frac{a_{1}}{2}$.

Proof of Claim 3. Assume $2 a<a_{1}$. Then, since $\left(X_{1}^{a} X_{2}^{b^{\prime}}\right)^{2} \in J^{2},\left(2 a, 2 b^{\prime}, 0\right)=$ $\beta+\lambda+a_{i} e_{i}$ for some $\beta \in L, \lambda \in \Lambda$, and $1 \leq i \leq 3$. As $2 a<a_{1}$, we get $i=2$, so that $\left(2 a, 2 b^{\prime}-a_{2}, 0\right)=\beta+\lambda$. Because $2 b^{\prime}-a_{2}<a_{2}$ and $\lambda_{3}=0$, we have $\lambda \in \Gamma_{3}$. Hence $\left(2 a, 2 b^{\prime}-a_{2}, 0\right)=\beta+\left(a, b^{\prime}, 0\right)$. Therefore $b^{\prime} \geq a_{2}$, which is impossible. Hence $2 a \geq a_{1}$, and so $a=\frac{a_{1}}{2}=a^{\prime}$ by Claim 1 , since $a+a^{\prime}=a_{1}$.

Claim 4. $c \geq \frac{a_{3}}{2} \geq c^{\prime}$.

Proof of Claim 4. We have $\left(X_{1}^{a^{\prime}} X_{3}^{c}\right)^{2}=X_{1}^{a_{1}} X_{2}^{2 c} \in J^{2}$. Hence $\left(a_{1}, 0,2 c\right)=\beta+\lambda+$ $a_{i} e_{i}$ for some $\beta \in L, \lambda \in \Lambda$, and $1 \leq i \leq 3$. Therefore, if $2 c<a_{3}$, we have $i=1$, so that $(0,0,2 c)=\beta+\lambda$. Hence $X_{3}^{2} c \in J$, so that $2 c \geq a_{3}$ by (8.8)(4). This is a contradiction. Thus $c \geq \frac{a_{3}}{2}$, whence $c \geq \frac{a_{3}}{2} \geq c^{\prime}$ as $c+c^{\prime}=a_{3}$.

Claim 5. $b=b^{\prime}=\frac{a_{2}}{2}$.

Proof of Claim 5. As $X_{1}^{a^{\prime}} X_{3}^{c} \cdot X_{2}^{b} X_{3}^{k}=X_{1}^{\frac{a_{1}}{2}} X_{2}^{b} X_{3}^{c+k} \in J^{2}$, we have $\left(\frac{a_{1}}{2}, b, c+k\right)$ $=\beta+\lambda+a_{i} e_{i}$ for some $\beta \in L, \lambda \in \Lambda$, and $1 \leq i \leq 3$. Then $i=3$ and $\left(\frac{a_{1}}{2}, b, c+k-a_{3}\right)$ $=\beta+\lambda$. Because $\lambda_{1} \leq \frac{a_{1}}{2}<a_{1}, \lambda_{2} \leq b<a_{2}$, and $\lambda_{3} \leq c+k-a_{3}<\min \{c, k\}<a_{3}$ (recall that $\max \{c, k\}<a_{3}$ ), we have $\lambda \in \Gamma$. As $\lambda_{3}<\min \{c, k\}$ and $\lambda_{2} \leq b<\ell$, we see that $\lambda \notin \Gamma_{1} \cup \Gamma_{2}$ whence $\lambda \in \Gamma_{3}$, so that $\lambda=\left(a, b^{\prime}, 0\right)$. Thus $b \geq \lambda_{2}=b^{\prime}$, and so $b=b^{\prime}=\frac{a_{2}}{2}$ by Claim 2 .

Claim 6. $c=c^{\prime}=\frac{a_{3}}{2}$.

Proof of Claim 6. As $X_{1}^{a} X_{2}^{b^{\prime}} \cdot X_{2}^{\ell} X_{3}^{c^{\prime}}=X_{1}^{\frac{a_{1}}{2}} X_{2}^{\frac{a_{2}}{2}+\ell} X_{3}^{c^{\prime}} \in J^{2}$, it follows that $\left(\frac{a_{1}}{2}, \frac{a_{2}}{2}\right.$ $\left.+\ell, c^{\prime}\right)=\beta+\lambda+a_{i} e_{i}$ for some $\beta \in L, \lambda \in \Lambda$, and $1 \leq i \leq 3$. Since $\frac{a_{1}}{2}<a_{1}$ and $c^{\prime}<a_{3}$, we have $i=2$, so that $\left(\frac{a_{1}}{2}, \ell-\frac{a_{2}}{2}, c^{\prime}\right)=\beta+\lambda$. Note that $\ell-\frac{a_{2}}{2}=\ell+b^{\prime}-a_{2}$ since $b^{\prime}=\frac{a_{2}}{2}$, and that $\ell+b^{\prime}-a_{2}<a_{2}$ since $\max \left\{\ell, b^{\prime}\right\}<a_{2}$. Then we have $\lambda \in \Gamma$, because $\lambda_{1} \leq \frac{a_{1}}{2}<a_{1}, \lambda_{2} \leq \ell-\frac{a_{2}}{2}<a_{2}$, and $\lambda_{3} \leq c^{\prime}<a_{3}$. We see $\lambda \notin \Gamma_{1}$, since $\lambda_{2} \leq \ell-\frac{a_{2}}{2}<\ell$ and $\lambda_{3} \leq c^{\prime}<k$. If $\lambda \in \Gamma_{3}$, then $\lambda=\left(a, b^{\prime}, 0\right)$, so that $\ell-\frac{a_{2}}{2} \geq \lambda_{2}=b^{\prime}=\frac{a_{2}}{2}$, whence $\ell \geq a_{2}$, which is impossible. Thus $\lambda=\left(a^{\prime}, 0, c\right)$ and we have $c^{\prime} \geq \lambda_{3}=c$, whence $c^{\prime}=c=\frac{a_{3}}{2}$ by Claim 4 .

Consequently we have $a=a^{\prime}=\frac{a_{1}}{2}, b=b^{\prime}=\frac{a_{2}}{2}$, and $c=c^{\prime}=\frac{a_{3}}{2}$. But then, since $X_{1}^{a} X_{2}^{b^{\prime}} \cdot X_{1}^{a^{\prime}} X_{3}^{c}=X_{1}^{a_{1}} X_{2}^{\frac{a_{2}}{2}} X_{3}^{\frac{a_{3}}{2}} \in J^{2}$, we must have $\left(a_{1}, \frac{a_{2}}{2}, \frac{a_{3}}{2}\right)=\beta+\lambda+a_{i} e_{i}$ for some $\beta \in L, \lambda \in \Lambda$, and $1 \leq i \leq 3$. Therefore $i=1$ and $\left(0, \frac{a_{2}}{2}, \frac{a_{3}}{2}\right)=\beta+\lambda$, so that $\lambda \in \Gamma_{1}$, whence $\lambda=\left(0, \frac{a_{2}}{2}, k\right)$ or $\lambda=\left(0, \ell, \frac{a_{3}}{2}\right)$. Thus $\left(0, \frac{a_{2}}{2}, \frac{a_{3}}{2}\right)=\beta+\left(0, \frac{a_{2}}{2}, k\right)$ or $\left(0, \frac{a_{2}}{2}, \frac{a_{3}}{2}\right)=\beta+\left(0, \ell, \frac{a_{3}}{2}\right)$. Hence $c^{\prime}=\frac{a_{3}}{2} \geq k$ or $b=\frac{a_{2}}{2} \geq \ell$, which is of course impossible. Thus $\mu_{R}(J) \neq 7$, which completes the proof of (8.19).

Contrary to Proposition (8.19), there exist however plenty of monomial good ideals $J$ with $\mu_{R}(J)=8$. Namely, 
Theorem (8.20). Let $n \geq 3$ be an integer and put $Q=\left(X_{1}^{2}, X_{2}^{3}, X_{3}^{n}\right)$. Let $J$ be a graded ideal in $R$. Then the following conditions are equivalent.

(1) $J \in \mathcal{X}_{Q}$.

(2) There exist integers $a, b, c>0$ satisfying the conditions $a+b=n, a+c \geq n$, $2 a \geq c$, and $2 n-a \geq 2 c \geq n+a$ such that

$$
J=Q+\left(X_{2} X_{3}^{c}, X_{2}^{2} X_{3}^{a}, X_{1} X_{3}^{b}, X_{1} X_{2}^{2}, X_{1} X_{2} X_{3}^{n-c}\right) .
$$

To prove Theorem (8.20) we need the following, where $Q=\left(X_{1}^{2}, X_{2}^{3}, X_{3}^{n}\right)$ with $n \geq 3$.

Lemma (8.21). Let $a, b, c, \ell \geq 1$ be integers satisfying the conditions $a+b=n$, $\ell \leq b, a \leq c<n$, and $b \leq c$. Let $K=Q+\left(X_{2} X_{3}^{c}, X_{2}^{2} X_{3}^{a}, X_{1} X_{3}^{b}, X_{1} X_{2}^{2}, X_{1} X_{2} X_{3}^{\ell}\right)$. Then

$$
Q: K=Q+\left(X_{2} X_{3}^{n-\ell}, X_{2}^{2} X_{3}^{a}, X_{1} X_{3}^{b}, X_{1} X_{2}^{2}, X_{1} X_{2} X_{3}^{n-c}\right) .
$$

Proof. Let

$$
\left\{\begin{array}{l}
I_{1}=\left(X_{1}, X_{2}, X_{3}^{n}\right) \\
I_{2}=\left(X_{1}^{2}, X_{2}^{2}, X_{3}^{n-c}\right) \\
I_{3}=\left(X_{1}^{2}, X_{2}, X_{3}^{n-a}\right)
\end{array}\right\} \quad\left\{\begin{array}{l}
I_{4}=\left(X_{1}, X_{2}^{3}, X_{3}^{n-b}\right) \\
I_{5}=\left(X_{1}, X_{2}^{2}, X_{3}^{n-\ell}\right)
\end{array}\right\} .
$$

Then $Q: K=\bigcap_{i=1}^{5} I_{i}$ by (8.4). Hence $Q: K=\left(X_{1}^{2}, X_{2}^{2}, X_{3}^{n}, X_{2} X_{3}^{n-c}, X_{1} X_{3}^{n-c}\right) \cap$ $I_{3} \cap I_{4} \cap I_{5}$, because $I_{1} \cap I_{2}=\left(X_{1}^{2}, X_{2}^{2}, X_{3}^{n}, X_{2} X_{3}^{n-c}, X_{1} X_{3}^{n-c}\right)$. Similarly

$$
\begin{aligned}
Q: K & =\left(X_{1}^{2}, X_{2}^{2}, X_{3}^{n}, X_{2} X_{3}^{n-c}, X_{1} X_{3}^{n-a}\right) \cap I_{4} \cap I_{5} \\
& =\left(X_{1}^{2}, X_{2}^{3}, X_{3}^{n}, X_{2} X_{3}^{n-b}, X_{1} X_{3}^{n-a}, X_{1} X_{2}^{2}, X_{1} X_{2} X_{3}^{n-c}\right) \cap I_{5} \\
& =Q+\left(X_{2} X_{3}^{n-\ell}, X_{2}^{2} X_{3}^{a}, X_{1} X_{3}^{b}, X_{1} X_{2}^{2}, X_{1} X_{2} X_{3}^{n-c}\right) .
\end{aligned}
$$

Proof of Theorem (8.20). (2) $\Rightarrow(1)$ Let $\ell=n-c$. Then $\ell>0$, as $2(n-c) \geq a$. Since $n-c \leq c-a \leq a$, we have $a+b=n \leq a+c$. Hence $b \leq c$. As $n=a+b$ and $\frac{n}{2} \geq a$, we have $b \geq \frac{n}{2} \geq a$. Hence $a \leq c$, and so $\ell=n-c \leq n-a=b$. Thus by (8.21) $Q: J=J$. It is standard to check that $J^{2}=Q J$, which we leave to the reader.

(1) $\Rightarrow(2)$ We look at the sets $\Gamma_{i}$ and $\Gamma^{\prime}$ in (8.10) for our ideal $J$. Then $\sharp \Gamma_{2}=$ $\sharp \Gamma_{3}=1$ by $(8.10)(3)(4)$ since $a_{1}=2$, and $\sharp \Gamma_{1}=2$ by $(8.10)(3)(4)$ and (8.16) since $a_{2}=3$. Therefore $\Gamma^{\prime} \neq \varnothing$, since $\mu_{R}(J) \geq 8$ by (8.19). Let $\Gamma_{1}=\{(0,1, c),(0,2, a)\}$ and $\Gamma_{2}=\{(1,0, b)\}$, where $a+b=n$ and $1 \leq a<c<n$ (cf. (8.10)(3)(4) and (8.12)). Hence $\Gamma_{3}=\{(1,2,0)\}$ by (8.12), and so we finally find that $\Gamma^{\prime}=\{(1,1, \ell)\}$ for some $1 \leq \ell<n$. Thus $J=Q+\left(X_{2} X_{3}^{c}, X_{2}^{2} X_{3}^{a}, X_{1} X_{3}^{b}, X_{1} X_{2}^{2}, X_{1} X_{2} X_{3}^{\ell}\right)$. We will show that these integers $a, b$, and $c$ satisfy the conditions stated in (2).

Claim 1. $2 c \geq n+a$.

Proof of Claim 1. Since $X_{2}^{2} X_{3}^{2 c} \in J^{2}$, by (8.8)(3) we have $(0,2,2 c)=\beta+\lambda+n e_{3}$ for some $\beta \in L$ and $\lambda \in \Lambda$. Hence $(0,2,2 c-n)=\beta+\lambda$, so that $\lambda=(0,2, a)$ because $2 c-n<c$. Thus $2 c-n \geq \lambda_{3}=a$.

Claim 2. $b \geq \frac{n}{2} \geq a$. 
Proof of Claim 2. Since $X_{1}^{2} X_{3}^{2 b} \in J^{2}$, we have $(2,0,2 b)=\beta+\lambda+a_{i} e_{i}$ for some $\beta \in L, \lambda \in \Lambda$, and $1 \leq i \leq 3$ (here $a_{1}=2, a_{2}=3$, and $a_{3}=n$ ). Hence if $2 b<n$, we get $i=1$ and $(0,0,2 b)=\beta+\lambda$. Therefore $\lambda=\lambda_{3} e_{3}$, and so $2 b \geq \lambda_{3}=n$ by (8.8)(2), which is absurd. Thus $b \geq \frac{n}{2}$ so that $\frac{n}{2} \geq a$ since $a+b=n$.

Claim 3. $a+c \geq n$. Hence $c \geq b \geq a$.

Proof of Claim 3. Since $X_{2}^{3} X_{3}^{a+c} \in J^{2}$, we have $(0,3, a+c)=\beta+\lambda+a_{i} e_{i}$ for some $\beta \in L, \lambda \in \Lambda$, and $1 \leq i \leq 3$. Hence if $a+c<n$, we get $i=2$ and $(0,0, a+c)=\beta+\lambda$. Therefore $\lambda=\lambda_{3} e_{3}$, and so $a+c \geq \lambda_{3}=n$ by (8.8)(2), which is absurd.

Claim 4. $2 a \geq c$.

Proof of Claim 4. By Claim 2 we have $2 a \leq n$. Therefore we may assume $2 a<n$, since $c<n$. Then, because $X_{2}^{4} X_{3}^{2 a} \in J^{2}$, we have $(0,4,2 a)=\beta+\lambda+a_{i} e_{i}$ for some $\beta \in L, \lambda \in \Lambda$, and $1 \leq i \leq 3$. Because $2 a<n$, we see that $i=2$ and $(0,1,2 a)=\beta+\lambda$. Hence $\lambda \in \Gamma_{1}$, so that $\lambda=(0,1, c)$. Thus $(0,1,2 a)=\beta+(0,1, c)$, whence $2 a \geq c$.

Claim 5. $b>\ell$.

Proof of Claim 5. Since $X_{1} X_{2} X_{3}^{b+c} \in J^{2}$, we have $(1,1, b+c)=\beta+\lambda+n e_{3}$ for some $\beta \in L$ and $\lambda \in \Lambda$. Hence $(1,1, b+c-n)=\beta+\lambda$. Because $b+c-n<\min \{b, c, n\}$ (recall that $\max \{b, c\}<n$ ), we have $\lambda \in \Gamma$. However, since $\lambda_{2} \leq 1$, we see that $\lambda \neq(0,2, a)$ and $\lambda \neq(1,2,0)$. Similarly, since $\lambda_{3} \leq b+c-n<\min \{b, c\}$, we see that $\lambda \neq(1,0, b)$ and $\lambda \neq(0,1, c)$. Hence $\lambda \in \Gamma^{\prime}$, so that $(1,1, b+c-n)=\beta+(1,1, \ell)$. Thus $b+c-n \geq \ell$, whence $b>\ell$ as $n>c$.

Consequently by (8.21) we have

$$
J=Q: J=Q+\left(X_{2} X_{3}^{n-\ell}, X_{2}^{2} X_{3}^{a}, X_{1} X_{3}^{b}, X_{1} X_{2}^{2}, X_{1} X_{2} X_{3}^{n-c}\right),
$$

whence $\Lambda=\{(1,2,0),(0,1, n-\ell),(0,2, a),(1,0, b),(1,1, n-c)\} \cup\left\{2 e_{1}, 3 e_{2}, n e_{3}\right\}$. Thus $c=n-\ell$. Finally, as $X_{1}^{2} X_{2}^{2} X_{3}^{2 \ell} \in J^{2}$, we have $(2,2,2 \ell)=\beta+\lambda+a_{i} e_{i}$ for some $\beta \in L, \lambda \in \Lambda$, and $1 \leq i \leq 3$. Hence if $2 \ell<n$, then $i=1$ and $(0,2,2 \ell)=\beta+\lambda$. Therefore $\lambda \in \Gamma_{1}$, and so $(0,2,2 \ell)=\beta+(0,1, c)$ or $(0,2,2 \ell)=\beta+(0,2, a)$. As $c>a$, we have $2 \ell \geq a$ in any case. Thus $2(n-c) \geq a$, as $\ell=n-c$, which completes the proof of Theorem (8.20).

Corollary (8.22). $\mathcal{X}_{(2,3, n)} \neq \varnothing$ if $n \geq 3$.

Proof. Let $\alpha \in \mathbb{Z}$ be the smallest integer such that $\alpha \geq \frac{2}{3} n$. We put $a=2 \alpha-n$, $b=n-a$, and $c=\alpha$. Then $\alpha<n$, since $\frac{2}{3} n>\alpha-1$ and $n \geq 3$. Therefore $b=n-a=2(n-\alpha) \geq 1$. We have $a=2 \alpha-n \geq \frac{1}{3} n>0$ and $c=\alpha \geq 2$. Thus $a, b, c \geq 1$ and $a+b=n$. Because $a+c=3 \alpha-n \geq n, 2 c=n+a$, and $2 a-c=3 \alpha-2 n \geq 0$, we get $a+c \geq n, 2 c \geq n+a$, and $2 a \geq c$. Now notice that $2 n-(a+2 c)=3 n-4 \alpha$, whence $2 n-a \geq 2 c$ if and only if $n \geq \frac{4}{3} \alpha$. Therefore, because $n>\frac{3}{2}(\alpha-1)$ and because $\frac{3}{2}(\alpha-1)-\frac{4}{3} \alpha=\frac{\alpha-9}{6}$, we have $2 n-a \geq 2 c$ if $\alpha \geq 9$. Assume that $\alpha \leq 8$. Then $n \leq 12$, and it is routine to check that $n \geq \frac{4}{3} \alpha$ for all $3 \leq n \leq 12$ but $n \neq 5$. Hence $\mathcal{X}_{(2,3, n)} \neq \varnothing$ by (8.21) if $n \geq 3$ and $n \neq 5$. For $n=5$ we have a unique solution $(a, b, c)=(2,3,4)$, and so $\mathcal{X}_{(2,3,5)} \neq \varnothing$ too.

Proposition (8.23). $\mathcal{X}_{(2,4, n)} \neq \varnothing$ if $n \geq 2$. 
Proof. By (8.7) and (8.15) we may assume $n=2 \ell+1(\ell \geq 1)$. We put

$$
J=Q+\left(X_{2}^{2} X_{3}^{\ell+1}, X_{2}^{3} X_{3}^{\ell}, X_{1} X_{3}^{\ell+1}, X_{1} X_{2}^{2}, X_{1} X_{2} X_{3}^{\ell}\right),
$$

where $Q=\left(X_{1}^{2}, X_{2}^{4}, X_{3}^{n}\right)$. Then it is direct to check that $J^{2}=Q J$ and $J=Q: J$ (see the proof of (8.21)). Hence $J \in \mathcal{X}_{Q}$.

Proposition (8.24). Let $\ell \geq 2$ be an integer. Then $\mathcal{X}_{(2,2 \ell+1, n)} \neq \varnothing$ if $n \geq \ell+2$.

Proof. Let us note the following examples only. The proof is standard, although it needs a lot of boring work. Let $X=X_{1}, Y=X_{2}$, and $Z=X_{3}$. Let $q \geq 1$ and $\ell \geq 2$ be integers, and let $Q=\left(X^{2}, Y^{2 \ell+1}, Z^{n}\right)$. Then for the following ideals $K$ the ideals $J=Q+K$ are $Q$-good:

(1) If $n=4 q+\ell-2$, let

$$
K=\left(X Y^{\ell+1}, Y^{\ell} Z^{n-q}, X Y^{\ell} Z^{q}\right)+Y^{\ell+1} Z^{2 q-1}(Y, Z)^{\ell-1}+X Z^{q}(Y, Z)^{q+\ell-1} .
$$

(2) If $n=4 q+\ell-1$ or $n=4 q+\ell$, let

$$
K=\left(X Y^{\ell+1}, Y^{\ell} Z^{n-q}, X Y^{\ell} Z^{q}\right)+Y^{\ell+1} Z^{2 q}(Y, Z)^{\ell-1}+X Z^{q}(Y, Z)^{n-3 q} .
$$

(3) If $n=4 q+\ell+1$, let

$$
\begin{aligned}
& K=\left(X Y^{\ell+1}, Y^{\ell} Z^{n-q-1}, X Y^{\ell} Z^{q+1}\right)+Y^{\ell+1} Z^{2 q+1}(Y, Z)^{\ell-1} \\
& +X Z^{q+1}(Y, Z)^{n-3 q-2} .
\end{aligned}
$$

We are now in a position to prove the main result (8.25) of this section. The theorem asserts that $\mathcal{X}_{Q} \neq \varnothing$ for almost all the monomial parameter ideals $Q=$ $\left(X_{1}^{a}, X_{2}^{b}, X_{3}^{c}\right)(a, b, c \geq 1)$ in the polynomial ring $R=k\left[X_{1}, X_{2}, X_{3}\right]$, so that the 3-dimensional regular local rings $A=R_{\mathfrak{M}}$ and $S=k\left[\left[X_{1}, X_{2}, X_{3}\right]\right]$ really contain plenty of good ideals.

Theorem (8.25). Let $a, b, c \geq 1$ be integers. Then $\mathcal{X}_{(a, b, c)}=\varnothing$ if and only if one of the following conditions is satisfied.

(1) $\{a, b, c\} \ni 1$.

(2) $2 \nmid a b c$.

(3) $(a, b, c)=(2,2$, odd $),(2$, odd, 2$)$, or $($ odd $, 2,2)$.

Our proof of Theorem (8.25) depends on the following.

Lemma (8.26). Let $m, n \geq 2$ be integers. Then $(2, m, n)=(2,2$, odd $)$ or $(2, m, n)$ $=(2$, odd, 2$)$ if $\mathcal{X}_{(2, m, n)}=\varnothing$.

Proof. Since $\mathcal{X}_{(2,2,2)} \neq \varnothing$, by (8.15) we may assume $m$ is odd. Let $m=2 \ell+1$ $(\ell \geq 1)$. If $\ell=1$, then $(2, m, n)=(2,3, n)$, so that $n=2$ by (8.22). Assume that $\ell \geq 2$. Then $n \leq \ell+1$ by (8.24). We furthermore have

Claim 1. $2 \mid n$.

Proof of Claim 1. Suppose that $n=2 \ell^{\prime}+1\left(\ell^{\prime} \geq 1\right)$. If $\ell^{\prime}=1$, then $n=3$ and $\mathcal{X}_{(2,2 \ell+1,3)}=\varnothing$. Hence by $(8.22)$ we must have $m=2 \ell+1 \leq 2$, which is absurd. Therefore $\ell^{\prime} \geq 2$, and so by (8.24) we get $m \leq \ell^{\prime}+1$, which is still impossible because

$$
m=2 \ell+1 \leq \ell^{\prime}+1<2 \ell^{\prime}+1=n \leq \ell+1 .
$$

Thus $n$ must be even.

Claim 2. $n=2$. 
Proof of Claim 2. Let $n=2 s$. Then $s$ is odd by (8.15), because $\mathcal{X}_{(2, m, 4)} \neq \varnothing$ by (8.23). Let $s=2 \ell^{\prime}+1$. We must show that $\ell^{\prime}=0$. If $\ell^{\prime}=1$, then $(2, m, n)=$ $(2, m, 6)$, so that $\mathcal{X}_{(2, m, 3)}=\varnothing$ by $(8.15)$, while $\mathcal{X}_{(2, m, 3)} \neq \varnothing$ by (8.22). Assume that $\ell^{\prime} \geq 2$. Then, because $\mathcal{X}_{\left(2, m, 2 \ell^{\prime}+1\right)}=\varnothing$ by $(8.15)$, we have $2 \mid m$ by Claim 1 , which is impossible. Thus $\ell^{\prime}=0$ and $n=2$.

This finishes the proof of Lemma (8.26).

Proof of Theorem (8.25). If $\{a, b, c\} \ni 1$ or $a b c$ is odd, then $\mathcal{X}_{(a, b, c)}=\varnothing$ by (8.6)(4) and (8.12). If $(a, b, c)=(2,2, o d d)$ or its permutation, we have $\mathcal{X}_{(a, b, c)}=\varnothing$ by (8.18). Conversely, let $a, b, c \geq 2$ be integers with $a b c$ even, and assume that $\mathcal{X}_{(a, b, c)}=\varnothing$. We may assume $a$ is even, say $a=2 k$. Then, since $\mathcal{X}_{(2, b, c)}=\varnothing$ by $(8.15)$, we have by $(8.26)(2, b, c)=(2,2, o d d)$ or $(2, b, c)=(2$, odd, 2$)$. Hence $b=2$ or $c=2$, so again by (8.26) we get $a=2$ too. This completes the proof of Theorem $(8.25)$.

Corollary (8.27). Let $a, b, c \geq 3$ be integers. Then $\mathcal{X}_{(a, b, c)} \neq \varnothing$ if and only if $2 \mid a b c$.

\section{REFERENCES}

[BH] W. Bruns and J. Herzog, Cohen-Macaulay rings, Cambridge studies in advanced mathematics, vol. 39, Cambridge University Press, 1993. MR 95h:13020

[G] J. Giraud, Improvement of Grauert-Riemenschneider's Theorem for a normal surface, Ann. Inst. Fourier 32 (1982), fasc. 4, 13-23. MR 84f:14025

[GH] S. Goto and S. Huckaba, On graded rings associated to analytic deviation one ideals, Amer. J. Math. 116 (1994), 905-919. [MR 95h:13003]

[GN] S. Goto and K. Nishida, The Cohen-Macaulay and Gorenstein Rees algebras associated to filtrations, Mem. Amer. Math. Soc. 110 (1994), no. 526. MR 95b:13001]

[GS] S. Goto and Y. Shimoda, On the Rees algebras of Cohen-Macaulay local rings, Commutative Algebra, Analytic Methods (R. N. Draper, ed.), Lecture Notes in Pure and Applied Mathematics, vol. 68, Marcel Dekker, Inc., New York and Basel, 1982, pp. 201-231. MR 84a: 13021

[GW] S. Goto and K. Watanabe, On graded rings I, J. Math. Soc. Japan 30 (1978), 179-213. MR 81m:13021

[H] C. Huneke, Hilbert functions and symbolic powers, Michigan Math. J. 34 (1987), 293-318. MR 89b:13037

[HE] M. Hochster and J. A. Eagon, Cohen-Macaulay rings, invariant theory, and the generic perfection of of determinantal loci, Amer. J. Math. 93 (1971), 1020-1058. MR 46:1787

[HHR] M. Herrmann, C. Huneke, and J. Ribbe, On reduction exponents of ideals with Gorenstein form rings, Proc. Edinburgh Math. Soc. 38 (1995), 449-463. MR 96i:13007

[HIO] M. Herrmann, S. Ikeda, and U. Orbanz, Equimultiplicity and blowing up, Springer-Verlag, 1988. MR 89g:13012

[HK] J. Herzog and E. Kunz (eds.), Der kanonische Modul eines Cohen-Macaulay-Rings, Lecture Notes in Mathematics, vol. 238, Springer-Verlag, 1971. MR 54:304

[HS] C. Huneke and I. Swanson, Cores of ideals in 2-dimensional regular local rings, Michigan Math. J. 42 (1995), 193-208. MR 96j:13021

[I] S. Ikeda, On the Gorensteinness of Rees algebras over local rings, Nagoya Math. J 102 (1986), 135-154. MR 87j:13031

[K] Ma. Kato, Riemann-Roch theorem for strongly pseudoconvex manifolds of dimension 2, Math. Ann. 222 (1976), 243-250. MR 54:594

[La] H. B. Laufer, On minimally elliptic singularities, Amer. J. Math. 99 (1977), 1257-1295. MR 58:27961

[L1] J. Lipman, Rational singularities, with applications to algebraic surfaces and unique factorization, Publ. Math. IHES 36 (1969), 195-279. MR 43:1986

[L2] J. Lipman, Stable ideals and Arf rings, Amer. J. Math. 93 (1971), 649-685. MR 44:203 
[L3] J. Lipman, Desingularization of two-dimensional schemes, Ann. of Math. 107 (1978), 151-207. MR 58:10924

[L4] J. Lipman, Adjoints of ideals in regular local rings, Mathematical Research Letters 1 (1994), 739-755. MR 95k:13028

[LT] J. Lipman and B. Teissier, Pseudo-rational local rings and a theorem of Briançon-Skoda about integral closures of ideals, Michigan Math. J. 28 (1981), 97-116. MR 82f:14004

[NR] D. G. Northcott and D. Rees, Reductions of ideals in local rings, Math. Proc. Cambridge Philos. Soc. 50 (1954), 145-158. MR 15:596a

[R] I. Reiten, The converse to a theorem of Sharp on Gorenstein modules, Proc. Amer. Math. Soc. 32 (1972), 417-420. MR 45:5128

[S1] J. D. Sally, On the associated graded ring of a local Cohen-Macaulay ring, J. Math. Kyoto Univ. 17 (1977), 19-21. MR 56:8555

[S2] J. Sally, Numbers of generators of ideals in local rings, Lecture Notes in Pure and Applied Mathematics, vol. 35, Marcel Dekker, Inc., New York and Basel, 1978. MR 58:5654

[Sh] K. Shah, On the Cohen-Macaulayness of the fiber cone of an ideal, J. Alg. 143 (1991), 156-172. MR 92k:13014

[VV] P. Valabrega and G. Valla, Form rings and regular sequences, Nagoya Math. J. 72 (1978), 93-101. MR 80d:14014

Department of Mathematics, School of Science and Technology, Meiji University, 214-8571 JAPAN

E-mail address: goto@math.meiji.ac.jp

Department of Mathematics, School of Science and Technology, Meiji University, 214-8571 JAPAN

E-mail address: s-iai@math.meiji.ac.jp

Department of Mathematics, Nihon University, 156-8550 Japan

E-mail address: watanabe@math.chs.nihon.-u.ac.jp 